MA XIAONAN

Formes de torsion analytique et familles des submersions I

Bulletin de la S. M. F., tome 127, no 4 (1999), p. 541-621

<http://www.numdam.org/item?id=BSMF_1999_127_4_541_0>

(C) Bulletin de la S. M. F., 1999, tous droits réservés.

L'accès aux archives de la revue «Bulletin de la S. M. F. » (http: //smf.emath.fr/Publications/Bulletin/Presentation.html) implique l'accord avec les conditions générales d'utilisation (http:/www.numdam.org/ conditions). Toute utilisation commerciale ou impression systématique est constitutive d'une infraction pénale. Toute copie ou impression de ce fichier doit contenir la présente mention de copyright.

\title{
Numdam
}

Article numérisé dans le cadre du programme

Numérisation de documents anciens mathématiques

http://www.numdam.org/ 
Bull. Soc. math. France,

127, 1999, p. 541-621.

\section{FORMES DE TORSION ANALYTIQUE \\ ET FAMILLES DE SUBMERSIONS I}

PAR XiAONAN MA (*)

RÉSUMÉ. - Soient $\pi_{1}: W \rightarrow V$ et $\pi_{2}: V \rightarrow S$ des submersions holomorphes de variétés complexes de fibre compacte. Soit $\xi$ un fibré holomorphe sur $W$. On démontre la fonctorialité des formes de torsion analytique relativement à la composition de deux submersions. Ce résultat étend une formule de Berthomieu-Bismut à une situation en famille.

Abstract. - AnAlytic torsion forms ANd families OF SUbMERsions. - Let $\pi_{1}: W \rightarrow V$ and $\pi_{2}: V \rightarrow S$ be holomorphic submersions of complex varieties with compact fibre. Let $\xi$ be a holomorphic vector bundle on $W$. We prove the functoriality of the analytic torsion forms with respect to the composition of two submersions. This result extends to a relative situation a result by Berthomieu-Bismut, proved in the case where $S$ is a point.

\section{Introduction}

Le but de ce travail est de démontrer la fonctorialité des formes de torsion analytique relativement à la composition de deux submersions; cet article est la première partie de ce travail.

Soient $W, V, S$ des variétés complexes. Soient $\pi_{1}: W \rightarrow V, \pi_{2}: V \rightarrow S$ des submersions holomorphes de fibres compactes $X, Y$. Alors $\pi_{3}=\pi_{2} \circ \pi_{1}: W \rightarrow S$ est une submersion holomorphe de fibre compacte $Z$.

Soit $\xi$ un fibré vectoriel holomorphe sur $W$. Soient $R^{\bullet} \pi_{1 *} \xi, R^{\bullet} \pi_{3 *} \xi, R^{\bullet} \pi_{2 *} R^{\bullet} \pi_{1 *} \xi$ les images directes de $\xi, \xi, R^{\bullet} \pi_{1 *} \xi$. On suppose que $R^{\bullet} \pi_{1 *} \xi, R^{\bullet} \pi_{3 *} \xi$ et $R^{\bullet} \pi_{2 *} R^{\bullet} \pi_{1 *} \xi$ sont localement libres.

(*) Texte recu le 27/10/98, revisé le 10/12/1998, accepté le 6/01/99.

X.MA, Université Paris-Sud, Département de Mathématiques, Bât. 425, 91405 Orsay CedeX (France).

Email xiaonan@topo.math.u-psud.fr.

Mots clés : faisceaux, cohomologie des sections de fibrés vectoriels holomorphes, classes caractéristiques, nombres caractéristiques, théorie de l'index, théorie du point fixe.

Classification AMS : 32L 10, 57 R 20, 58 G 10.

BULLETIN DE LA SOCIÉTÉ MATHÉMATIQUE DE FRANCE

(C) Société mathématique de France

$0037-9484 / 1999 / 541 / \$ 5.00$ 
Soient $H\left(Z, \xi_{\mid Z}\right), H\left(X, \xi_{\mid X}\right)$ les cohomologies de $\xi_{\mid Z}, \xi_{\mid X}$. Alors $H\left(Z, \xi_{\mid Z}\right)$ est un fibré holomorphe $\mathbb{Z}$-gradué sur $S$. Plus exactement, on a

$$
R^{\bullet} \pi_{3 *} \xi=H\left(Z, \xi_{\mid Z}\right)
$$

De même, on a $R^{\bullet} \pi_{1 *} \xi=H\left(X, \xi_{\mid X}\right)$.

Soit $\omega^{V}$ (resp. $\omega^{W}$ ) une $(1,1)$-forme réelle, fermée sur $V$ (resp. $W$ ), dont la restriction à chaque fibre $Y$ (resp. $Z$ ) définit une métrique hermitienne $g^{T Y}$ (resp. $g^{T Z}$ ) sur le fibré tangent relatif $T Y$ (resp. $T Z$ ). Soit $g^{T X}$ la métrique hermitienne sur $T X$ induite par $\omega^{W}$. Soit $h^{\xi}$ une métrique hermitienne sur $\xi$.

Soit $\left(\Omega\left(X, \xi_{\mid X}\right), \bar{\partial}^{X}\right)$ le complexe de Dolbeault des formes $\mathcal{C}^{\infty}$ sur la fibre $X$ à valeurs dans $\xi$. On munit $\Omega\left(X, \xi_{\mid X}\right)$ de la métrique hermitienne $L_{2}$ associée à $g^{T X}, h^{\xi}$. On identifie $H\left(X, \xi_{\mid X}\right)$ aux formes harmoniques dans le complexe $\Omega\left(X, \xi_{\mid X}\right)$. Soit $h^{H(X, \xi \mid X)}=h^{R \pi_{1 *} \xi}$ la métrique $L_{2}$ associée sur $H\left(X, \xi_{\mid X}\right)=$ $R^{\bullet} \pi_{1 *} \xi$.

De même, on considère les complexes de Dolbeault relatifs $\Omega\left(Z, \xi_{\mid Z}\right)$ et $\Omega\left(Y, R^{\bullet} \pi_{1 *} \xi_{\mid Y}\right)$ associés aux métriques $g^{T Z}, h^{\xi}$ et $g^{T Y}, h^{R \pi_{1 *} \xi}$. On désigne par $h^{H(Z, \xi \mid Z)}=h^{R \pi_{3 *} \xi}$ et $h^{R \pi_{2 *} R \pi_{1 *} \xi}$ les métriques $L_{2}$ correspondantes sur $H\left(Z, \xi_{\mid Z}\right)=R^{\bullet} \pi_{3 *} \xi, R^{\bullet} \pi_{2 *} R^{\bullet} \pi_{1 *} \xi$.

Soient $T_{1}\left(\omega^{W}, h^{\xi}\right), T_{2}\left(\omega^{V}, h^{R \pi_{1 *} \xi}\right)$ et $T_{3}\left(\omega^{W}, h^{\xi}\right)$ les formes de torsion analytique construites dans Bismut-Köhler [BKö] sur $V, S, S$ associées à $\left(\pi_{1}, \omega^{W}, h^{\xi}\right)$, $\left(\pi_{2}, \omega^{V}, h^{R \pi_{1 *} \xi}\right)$ et $\left(\pi_{3}, \omega^{W}, h^{\xi}\right)$. Les formes $T_{1}$ vérifient l'équation

$$
\frac{\bar{\partial} \partial}{2 \pi i} T_{1}\left(\omega^{W}, h^{\xi}\right)=\operatorname{ch}\left(R^{\bullet} \pi_{1 *} \xi, h^{R \pi_{1 *} \xi}\right)-\int_{X} \operatorname{Td}\left(T X, g^{T X}\right) \operatorname{ch}\left(\xi, h^{\xi}\right)
$$

et les formes $T_{2}$ et $T_{3}$ vérifient des équations analogues.

Soit $\widetilde{\mathrm{Td}}\left(T Z, T Y, g^{T Z}, g^{T Y}\right) \in P^{W} / P^{W, 0}$ la classe de Bott-Chern de [BGS1] telle que

$$
\begin{aligned}
\frac{\bar{\partial} \partial}{2 \pi i} \widetilde{\mathrm{Td}}\left(T Z, T Y, g^{T Z}, g^{T Y}\right) & \\
& =\operatorname{Td}\left(T Z, g^{T Z}\right)-\pi_{1}^{*}\left(\operatorname{Td}\left(T Y, g^{T Y}\right)\right) \operatorname{Td}\left(T X, g^{T X}\right)
\end{aligned}
$$

Pour $s \in S$, il existe une suite spectrale de Leray $\left(E_{r, s}, \mathrm{~d}_{r, s}\right), r \geq 2$, [Grot] telle que

$$
E_{2}=R^{\bullet} \pi_{2 *} R^{\bullet} \pi_{1 *} \xi
$$

Soit $h^{E_{2}}$ la métrique sur $E_{2}$ induite par $h^{R \pi_{2 *} R \pi_{1 *} \xi}$. On définit une classe de Bott-Chern

$$
\widetilde{\operatorname{ch}}\left(E_{2}, H\left(Z, \xi_{\mid Z}\right), h^{E_{2}}, h^{H\left(Z, \xi_{\mid Z}\right)}\right) \in P^{S} / P^{S, 0}
$$

TOME $127-1999-\mathrm{N}^{\circ} 4$ 
dans les trois cas suivants :

i) lorsque le fibré holomorphe $\xi$ est $\pi_{1 *}$ et $\pi_{3 *}$ acyclique.

ii) lorsque le rang des $E_{r}(r \geq 2)$ est localement constant sur $S$.

iii) lorsque $\pi_{1}$ et $V$ sont projectives.

On vérifie que ces définitions sont compatibles.

La classe $\widetilde{c h}\left(E_{2}, H\left(Z, \xi_{\mid Z}\right), h^{E_{2}}, h^{H\left(Z, \xi_{\mid Z}\right)}\right)$ vérifie l'équation

$$
\begin{aligned}
\frac{\bar{\partial} \partial}{2 \pi i} \widetilde{\operatorname{ch}}\left(E_{2}, H\left(Z, \xi_{\mid Z}\right), h^{E_{2}}, h^{H\left(Z, \xi_{\mid Z}\right)}\right) & \\
= & \operatorname{ch}\left(H\left(Z, \xi_{\mid Z}\right), h^{H\left(Z, \xi_{\mid Z}\right)}\right)-\operatorname{ch}\left(E_{2}, h^{E_{2}}\right)
\end{aligned}
$$

Le but de cet article est de montrer le théorème suivant.

ThÉorème 0.1. - Dans $P^{S} / P^{S, 0}$, on a l'identité

$$
\begin{aligned}
& \text { (0.4) } T_{3}\left(\omega^{W}, h^{\xi}\right)-T_{2}\left(\omega^{V}, h^{R \pi_{1 *} \xi}\right)-\int_{Y} \operatorname{Td}\left(T Y, g^{T Y}\right) T_{1}\left(\omega^{W}, h^{\xi}\right) \\
& +\int_{Z} \widetilde{\operatorname{Td}}\left(T Z, T Y, g^{T Z}, g^{T Y}\right) \operatorname{ch}\left(\xi, h^{\xi}\right)-\widetilde{\operatorname{ch}}\left(E_{2}, H\left(Z, \xi_{\mid Z}\right), h^{E_{2}}, h^{H\left(Z, \xi_{\mid Z}\right)}\right)=0 .
\end{aligned}
$$

On remarque que quand $S$ est un point, le théorème 0.1 est exactement [BerB, th. 3.1]. Dans [BerB], le résultat est énoncé comme une formule qui compare les métriques de Quillen sur $\operatorname{det} R^{\bullet} \pi_{3 *} \xi \simeq \operatorname{det} R^{\bullet} \pi_{2 *} R^{\bullet} \pi_{1 *} \xi$.

Supposons maintenant que $W, V, S$ sont des variétés arithmétiques. On rappelle que les images directes de Gillet et Soulé $\pi_{1 !}, \pi_{2 !}, \pi_{3}$ ! contiennent les formes de torsion analytique $T_{1}, T_{2}, T_{3}$. Le théorème 0.1 implique alors qu'on a la formule

$$
\pi_{3 !}\left(\xi, h^{\xi}\right)-\pi_{2 !}\left(\pi_{1 !}\left(\xi, h^{\xi}\right)\right)=-\int_{Z} \widetilde{\mathrm{Td}}\left(T Z, T Y, g^{T Z}, g^{T Y}\right) \operatorname{ch}\left(\xi, h^{\xi}\right)
$$

La formule (0.5) est un analogue d'un résultat de Faltings [F, lemme 5.5], dont la preuve est donnée dans [F, p. 75-76].

Cet article est organisé de la façon suivante.

- Au paragraphe 1, on calcule l'asymptotique de certains objets géométriques associés à une famille de submersions.

- Au paragraphe 2, on rappelle des résultats de [BGS2] et [BKö] sur les formes de torsion analytique.

- Au paragraphe 3 , on énonce le théorème 0.1 dans les cas i) et iii). 
- Au paragraphe 4 , on énonce sept résultats intermédiaires, dont les preuves sont différées aux $\S \S 5-9$. En utilisant ces résultats, on montre le théorème 0.1 dans le cas i).

Les paragraphes 5-9 sont consacrés aux preuves des résultats intermédiaires qui étendent [BerB, §5-9].

- Au paragraphe 5 , on calcule l'asymptotique des supertraces évaluées à l'aide d'une superconnexion qui dépend de deux paramètres $u$ et $T$.

- Au paragraphe 6 , on établit une égalité triviale pour la classe de Bott-Chern

$$
\widetilde{\operatorname{ch}}\left(E_{2}, H\left(Z, \xi_{\mid Z}\right), h^{E_{2}}, h^{H\left(Z, \xi_{\mid Z}\right)}\right) \in P^{S} / P^{S, 0}
$$

dans le cas i) pour lequel $H\left(Z, \xi_{\mid Z}\right)=E_{2}$.

Les paragraphes 7-9 contiennent des résultats de théorie de l'indice local relatif qu'on utilise pour montrer le théorème 0.1 .

- Au paragraphe 10, on construit la classe de Bott-Chern

$$
\widetilde{\operatorname{ch}}\left(E_{2}, H\left(Z, \xi_{\mid Z}\right), h^{E_{2}}, h^{H\left(Z, \xi_{\mid Z}\right)}\right) \in P^{S} / P^{S, 0}
$$

dans le cas où $\pi_{1}$ et $V$ sont projectives.

- Au paragraphes 11 et 12 , on montre le théorème 0.1 dans le cas iii).

La preuve du théorème 0.1 dans le cas ii) paraîtra comme partie II dans [Ma1].

Les résultats de cet article ont été annoncés dans [Ma]. On utilise le formalisme de superconnexion de Quillen [Q2]; pour les détails, on se réfèrera aussi à [B1], [BGS1], [BGS2], [BerB].

Remerciements. - Cet article est une partie de ma thèse de doctorat de l'Université Paris XI (Orsay). Je tiens à exprimer ma plus profonde gratitude à mon directeur de thèse, le professeur J.-M. Bismut. Il m'a soutenu tout au long de ce travail, du français aux mathématiques. Sans ses utiles discussions et suggestions, cet article n'aurait jamais été écrit. Je remercie aussi vivement le professeur Christophe Soulé pour ses observations.

\section{Fibrations kählériennes et limites adiabatiques}

Soient $\pi_{1}: W \rightarrow V$ et $\pi_{2}: V \rightarrow S$ des submersions de variétés $\mathcal{C}^{\infty}$ de fibres compactes $X, Y$. Alors $\pi_{3}=\pi_{2} \circ \pi_{1}: W \rightarrow S$ est une submersion $\mathcal{C}^{\infty}$ de fibre compacte $Z$.

Soient $\tilde{g}^{T W}, g^{T V}$ des métriques sur $W, V$. Pour $T>0$, on pose

$$
g_{T}^{T W}=\frac{1}{T^{2}} \tilde{g}^{T W}+\pi_{1}^{*} g^{T V} .
$$

TOME $127-1999-\mathrm{N}^{\circ} 4$ 
Par une construction de [BGS2, §1], la métrique $g_{T}^{T W}$ détermine un sous-fibré $T_{3, T}^{H} W$ de $T W$, une connexion $\nabla_{T}^{T Z}$, et des tenseurs $S_{3, T}$ et $T_{3, T}$.

Dans ce paragraphe, on étudie la limite quand $T$ tend vers $+\infty$ de ces différents objets. Ce paragraphe étend les résultats de $[\mathrm{BerB}, \S 7 \mathrm{~b})]$ obtenus dans le cas où $S$ est un point.

En a), on calcule l'asymptotique des objects définis.précédemment. En b), on applique les résultats de a) dans le cas des fibrations kählériennes (voir [BGS2, $\S 1])$.

\section{a) Fibrations et limites adiabatiques.}

Soient $W, V, S$ des variétés $\mathcal{C}^{\infty}$. Soient $\pi_{1}: W \rightarrow V, \pi_{2}: V \rightarrow S$ des submersions $\mathcal{C}^{\infty}$ de fibres compactes $X, Y$. Alors $\pi_{3}=\pi_{2} \circ \pi_{1}: W \rightarrow S$ est une submersion $\mathcal{C}^{\infty}$ de fibre compacte $Z$. On a donc

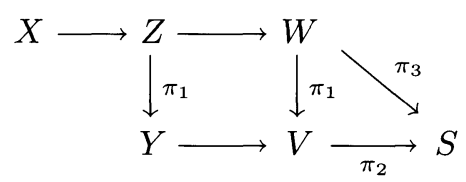

Soient $T Z=T W / S, T X, T Y$ les fibrés tangents relatifs. Soient $\tilde{g}^{T W}, g^{T V}$ des métriques sur $W, V$ et $\tilde{g}^{T Z}, g^{T X}, g^{T Y}$ les métriques induites par $\tilde{g}^{T W}, g^{T V}$ sur $T Z, T X, T Y$. Soit $T_{1}^{H} W$ (resp. $T_{2}^{H} V$ ) le sous-fibré de $T W$ orthogonal à $T X$ pour $\tilde{g}^{T W}$ (resp. $T V$ orthogonal à $T Y$ pour $g^{T V}$ ).

Soient $T_{1}, S_{1},\left\langle S_{1}(\cdot) \cdot, \cdot\right\rangle$ (resp. $T_{2}, S_{2},\left\langle S_{2}(\cdot) \cdot, \cdot\right\rangle$ ) les tenseurs définis en $[\mathrm{B} 4, \S 1.1]$ associés à $\left(\pi_{1}, g^{T X}, T_{1}^{H} W\right)\left(\operatorname{resp} .\left(\pi_{2}, g^{T Y}, T_{2}^{H} V\right)\right)$.

Pour $T>0$, on pose

$$
g_{T}^{T W}=\frac{1}{T^{2}} \tilde{g}^{T W}+\pi_{1}^{*} g^{T V}, \quad g^{T W}=g_{1}^{T W} .
$$

Soit $g_{T}^{T Z}$ la métrique induite par $g_{T}^{T W}$ sur $T Z$. Soit $T_{3, T}^{H} W$ le sous-fibré de $T W$ orthogonal à $T Z$ pour $g_{T}^{T W}$. Soient $T_{3, T}, S_{3, T},\left\langle S_{3, T}(\cdot) \cdot, \cdot\right\rangle_{T}$ les tenseurs définis en [B4, §1.1] associés à $\left(\pi_{3}, g_{T}^{T Z}, T_{3, T}^{H} W\right)$.

Soient $\nabla^{T X}, \nabla^{T Y}, \nabla_{T}^{T Z}$ les connexions sur $\left(T X, g^{T X}\right),\left(T Y, g^{T Y}\right),\left(T Z, g_{T}^{T Z}\right)$ associées à $\left(\pi_{1}, g^{T X}, T_{1}^{H} W\right),\left(\pi_{2}, g^{T Y}, T_{2}^{H} V\right),\left(\pi_{3}, g_{T}^{T Z}, T_{3, T}^{H} W\right)$ (voir [B1, §1c)] et $[\mathrm{B} 4, \S 1.1])$.

Le but de cette partie est de décrire l'asymptotique de

$$
\nabla_{T}^{T Z}, T_{3, T},\left\langle S_{3, T}(\cdot) \cdot, \cdot\right\rangle_{T} \quad \text { quand } T \rightarrow \infty .
$$

Si $S$ est un point, on a déjà étudié cette question dans le cadre complexe dans [BerB, $\S 4$ et $\S 7]$. 
On pose :

$$
T^{H} Z=T Z \cap T_{1}^{H} W .
$$

Alors on a les identifications de fibrés vectoriels $\mathcal{C}^{\infty}$ sur $W$,

$$
T Z \simeq T^{H} Z \oplus T X, \quad T^{H} Z \simeq \pi_{1}^{*} T Y .
$$

Les fibrés $T^{H} Z$ et $T X$ sont orthogonaux pour $\tilde{g}^{T Z}$. Soit $P^{T^{H} Z}$ (resp. $P^{T X}$ ) la projection de $T Z=T^{H} Z \oplus T X$ sur $T^{H} Z$ (resp. $T X$ ). Soit $g^{T^{H} Z}$ la métrique sur $T^{H} Z$ induite par $g^{T Y}$.

On pose :

$$
T_{3, \infty}^{H} W=\left\{X \in T_{1}^{H} W, \quad \pi_{1 *} X \in T_{2}^{H} V\right\}
$$

On a :

$$
T_{1}^{H} W=T_{3, \infty}^{H} W \oplus T^{H} Z .
$$

Les fibrés $T_{3, \infty}^{H} W$ et $T^{H} Z$ sont orthogonaux pour la métrique $\pi_{1}^{*} g^{T V}$.

Pour $U \in T S$, soit $U_{3, T}^{H} \in T_{3, T}^{H} W(1 \leq T \leq+\infty)$ (resp. $U_{2}^{H} \in T_{2}^{H} V$ ) le relèvement de $U$ dans $T_{3, T}^{H} W\left(\operatorname{resp} . T_{2}^{H} V\right.$ ) qui est tel que $\pi_{3 *} U_{3, T}^{H}=U$ (resp. $\left.\pi_{2 *} U_{2}^{H}=U\right)$. Pour $U \in T V$, soit $U_{1}^{H} \in T_{1}^{H} W$ le relèvement de $U$ dans $T_{1}^{H} W$ qui est tel que $\pi_{1 *} U_{1}^{H}=U$. On remarque que pour $U \in T S$,

$$
U_{3, \infty}^{H}=\left(U_{2}^{H}\right)_{1}^{H} \text {. }
$$

DÉfinition 1.1. - Soient $h \in \operatorname{End}\left(T^{H} Z\right), h^{\prime} \in \operatorname{Hom}\left(T_{3, \infty}^{H} W, T^{H} Z\right)$ tels que si $U, V \in T Y, X \in T_{2}^{H} V$, alors

$$
\left\{\begin{array}{l}
\left\langle U_{1}^{H}, V_{1}^{H}\right\rangle_{\tilde{g}^{T Z}}=\left\langle h U_{1}^{H}, V_{1}^{H}\right\rangle_{g^{T^{H} Z}}, \\
\left\langle X_{1}^{H}, V_{1}^{H}\right\rangle_{\tilde{g}^{T W}}=\left\langle h^{\prime} X_{1}^{H}, V_{1}^{H}\right\rangle_{g^{T^{H} Z}} .
\end{array}\right.
$$

Soient $g^{T S}, g^{\prime T S}$ des métriques sur TS. Notons que par [BGS2, rem. 1.6] et par (1.7), les objets introduits précédemment sont inchangés quand on remplace $\tilde{g}^{T W}$ et $g^{T V}$ par $\tilde{g}^{T W}+\pi_{3}^{*} g^{T S}$ et $g^{T V}+\pi_{2}^{*} g^{\prime S S}$.

Proposition 1.2. - Si $U \in T S, 1 \leq T<+\infty$, on a l'identité suivante dans $T W$ :

$$
U_{3, T}^{H}=U_{3, \infty}^{H}-\frac{1}{T^{2}}\left(1+\frac{h}{T^{2}}\right)^{-1} h^{\prime} U_{3, \infty}^{H} .
$$

Preuve. - Par définition, on a $T_{3, T}^{H} W \subset T_{1}^{H} W$. Donc il existe $U(T)$ appartenant à $T V$ tel que $U_{3, T}^{H}=U(T)_{1}^{H}$. D'où

$$
U(T)-U_{2}^{H} \in T Y .
$$

En utilisant (1.6), (1.7), (1.9), on en déduit facilement (1.8). 
Maintenant, on étudie l'asymptotique de $\nabla_{T}^{T Z}, T_{3, T},\left\langle S_{3, T}(\cdot) \cdot, \cdot\right\rangle_{T}$.

Soit $B_{T}(T \in[1,+\infty])$ une famille de tenseurs; quand $T$ tend vers $+\infty$, on notera

$$
B_{T}=O\left(\frac{1}{T^{2}}\right)
$$

si, pour tout $k \in \mathbb{N}, K \subset S$ compact, il existe $c>0$ tel que pour $T \geq 1$, le sup des normes de $B_{T}$ et de ses dérivées d'ordre $\leq k$ sur $K$ est dominé par $c T^{-2}$.

Soit $\nabla^{T^{H} Z}$ la connexion sur $T^{H} Z$ induite par $\nabla^{T Y}$; soit ${ }^{0} \nabla^{T Z}$ la connexion sur $T Z \simeq T^{H} Z \oplus T X$

$$
{ }^{0} \nabla^{T Z}=\nabla^{T^{H} Z} \oplus \nabla^{T X}
$$

Sur chaque fibre $Z$, soit $\widetilde{\nabla}^{T Z}$ la connexion de Levi-Civita $\operatorname{sur}\left(T Z, \tilde{g}^{T Z}\right)$. Soit $g^{\prime T Z}=\pi_{1}^{*} g^{T Y} \oplus g^{T X}$ la métrique sur $T Z=T^{H} Z \oplus T X$.

Définition 1.3. - Sous l'identification $T W=T_{3, \infty}^{H} W \oplus T Z$, soit $A_{3, \infty}$ la 1-forme sur $W$ à valeurs dans $\operatorname{End}(T Z)$ définie par

$$
\left\{\begin{array}{c}
\left\langle A_{3, \infty}(X) Y, Z\right\rangle_{g^{\prime T Z}}=\left\langle\left(\widetilde{\nabla}^{T Z}-{ }^{0} \nabla^{T Z}\right)(X) Y, P^{T X} Z\right\rangle_{g^{T X}} \\
\text { si } X, Y, Z \in T Z \\
=\frac{1}{2}\left\{\left\langle\left[X, P^{T^{H} Z} Y\right], P^{T X} Z\right\rangle_{g^{T X}}+\left\langle\left[h^{\prime} X, P^{T X} Z\right], P^{T^{H}} Z Y\right\rangle_{\pi_{1}^{*} g^{T Y}}\right\} \\
\text { si } X \in T_{3, \infty}^{H} W \text { et } Y, Z \in T Z .
\end{array}\right.
$$

On pose

$$
\nabla_{\infty}^{T Z}={ }^{0} \nabla^{T Z}+A_{3, \infty}
$$

ThÉORÈme 1.4. - La connexion $\nabla_{\infty}^{T Z}$ préserve $T X$ et sa restriction à $T X$ est égale $\grave{a} \nabla^{T X}$. Quand $T$ tend vers $+\infty$, on a

$$
\nabla_{T}^{T Z}=\nabla_{\infty}^{T Z}+O\left(\frac{1}{T^{2}}\right)
$$

Preuve. - Si $Y, Z$ sont des sections $\mathcal{C}^{\infty}$ de $T Z$, pour $X \in T W$, on doit calculer l'asymptotique de $\left\langle\nabla_{T, X}^{T Z} Y, Z\right\rangle_{g^{\prime T Z}}$ quand $T \rightarrow+\infty$.

Soient $Y_{1}, Z_{1}$ (resp. $Y_{2}, Z_{2}$ ) des sections $\mathcal{C}^{\infty}$ de $T Y$ (resp. $T X$ ). Soient $Y=Y_{1,1}^{H}+Y_{2}, Z=Z_{1,1}^{H}+Z_{2}$.

i) Le cas où $X \in T Z$. - Soient $X_{1}, X_{2}$ des sections $\mathcal{C}^{\infty}$ de $T Y, T X$. Soit $X=X_{1,1}^{H}+X_{2}$. Par (1.1), on a

$$
\begin{aligned}
& \left\langle\nabla_{T, X}^{T Z} Y, Z\right\rangle_{g^{\prime T Z}} \\
& \quad=T^{2}\left\langle\nabla_{T, X}^{T Z} Y, Z_{2}\right\rangle_{g_{T}^{T Z}}+\left\langle\nabla_{T, X}^{T Z} Y, Z_{1,1}^{H}\right\rangle_{g_{T}^{T Z}}\left(1+O\left(\frac{1}{T^{2}}\right)\right) .
\end{aligned}
$$

BULLETIN DE LA SOCIÉTÉ MATHÉMATIQUE DE FRANCE 
En utilisant la formule de la connexion de Levi-Civita [BeGeV, (1.18)], [B4, th. 1.1] et (1.14), on a

$$
\left\langle\nabla_{T, X}^{T Z} Y, Z\right\rangle_{g^{\prime T Z}}=\left\langle\widetilde{\nabla}_{X}^{T Z} Y, Z_{2}\right\rangle_{g^{T X}}+\left\langle\nabla_{X}^{T Y} Y_{1}, Z_{1}\right\rangle_{g^{T Y}}+O\left(\frac{1}{T^{2}}\right) .
$$

ii) Le cas où $X \in T_{3, \infty}^{H} W$. - Soit $U$ une section $\mathcal{C}^{\infty}$ de $T S$. Soit $X=U_{3, \infty}^{H}$.

- Supposons d'abord que $Z \in T X$. Alors $Z_{1}=0$. Par [B4, th. 1.1], (1.1) et $(1.8)$, on a

$$
\begin{array}{r}
2\left\langle\nabla_{T, U_{3, \infty}^{H}}^{T Z} Y, Z\right\rangle_{g^{\prime} T Z}=2 T^{2}\left\langle\nabla_{T, U_{3, T}^{H}}^{T Z} Y, Z\right\rangle_{g_{T}^{T Z}}+O\left(\frac{1}{T^{2}}\right) \\
=T^{2}\left\{\left\langle\left[U_{3, T}^{H}, Y\right], Z\right\rangle_{g_{T}^{T Z}}+\left\langle\left[Z, U_{3, T}^{H}\right], Y\right\rangle_{g_{T}^{T Z}}\right. \\
\left.+U_{3, T}^{H}\langle Y, Z\rangle_{g_{T}^{T Z}}\right\}+O\left(\frac{1}{T^{2}}\right) \\
=2\left\langle\nabla_{U_{3, \infty}^{H}}^{T X} Y_{2}, Z\right\rangle_{g^{T X}}+2\left\langle A_{3, \infty}\left(U_{3, \infty}^{H}\right) Y, Z\right\rangle_{g^{\prime} T Z}+O\left(\frac{1}{T^{2}}\right) .
\end{array}
$$

- Supposons que $Z \in T_{1}^{H} W$. Alors $Z_{2}=0$. En utilisant [B4, th. 1.1] et (1.8), on obtient :

$$
\left\langle\nabla_{T, U_{3, \infty}^{H}}^{T Z} Y, Z_{1,1}^{H}\right\rangle_{g_{T}^{T Z}}=\left\langle\nabla_{U_{2}^{H}}^{T Y} Y_{1}, Z_{1}\right\rangle_{g^{T Y}}+O\left(\frac{1}{T^{2}}\right)
$$

Nous avons bien démontré le théorème 1.4.

Soit $g^{T S}$ une métrique sur $T S$, soit $\nabla^{T S}$ la connexion de Levi-Civita sur $\left(T S, g^{T S}\right)$. Soient $g^{T_{3, \infty}^{H} W}$ la métrique et $\nabla^{T_{3, \infty}^{H} W}$ la connexion sur $T_{3, \infty}^{H} W$ induites par $g^{T S}, \nabla^{T S}$. Soit ${ }^{0} \nabla_{\infty}^{T W}$ la connexion sur $T W=T_{3, \infty}^{H} W \oplus T Z$

$$
{ }^{0} \nabla_{\infty}^{T W}=\nabla^{T_{3, \infty}^{H} W} \oplus \nabla_{\infty}^{T Z}
$$

et soit $T_{3, \infty}$ la torsion de ${ }^{0} \nabla_{\infty}^{T W}$.

Proposition 1.5. - On a

$$
T_{3, \infty}(X, Y)= \begin{cases}T_{1}(X, Y)+\left[T_{2}\left(\pi_{1 *} X, \pi_{1 *} Y\right)\right]_{1}^{H}+A_{3, \infty}(X) Y \\ \text { si } X \in T_{3, \infty}^{H} W, Y \in T W \\ 0 & \text { si } X, Y \in T Z\end{cases}
$$

Preuve. - On fait le calcul dans les cas suivants.

TOME $127-1999-\mathrm{N}^{\circ} 4$ 
(i) Le cas où $X, Y \in T_{3, \infty}^{H} W$. - Soient $U, U^{\prime}$ des sections $\mathcal{C}^{\infty}$ de $T S$; alors :

$$
\begin{aligned}
T_{3, \infty}\left(U_{3, \infty}^{H}, U_{3, \infty}^{\prime H}\right) & =\nabla_{U_{3, \infty}^{H}}^{T_{3, \infty}^{H} W} U_{3, \infty}^{\prime H}-\nabla_{U_{3, \infty}^{\prime H}}^{T_{3, \infty}^{H} W} U_{3, \infty}^{H}-\left[U_{3, \infty}^{H}, U_{3, \infty}^{\prime H}\right] \\
& =\left\{\left[U, U^{\prime}\right]\right\}_{3, \infty}^{H}-\left[U_{3, \infty}^{H}, U_{3, \infty}^{\prime H}\right] \\
& =T_{1}\left(U_{3, \infty}^{H}, U_{3, \infty}^{\prime H}\right)+\left[T_{2}\left(U_{2}^{H}, U_{2}^{\prime H}\right)\right]_{1}^{H}
\end{aligned}
$$

(ii) Le cas où $X \in T_{3, \infty}^{H} W, Y \in T X$. - Soit $U$ (resp. $Y$ ) une section $\mathcal{C}^{\infty}$ de $T S$ (resp. $T X$ ); alors, par (1.13),

$$
T_{3, \infty}\left(U_{3, \infty}^{H}, Y\right)=\nabla_{U_{3, \infty}^{H}}^{T X} Y-\left[U_{3, \infty}^{H}, Y\right]=T_{1}\left(U_{3, \infty}^{H}, Y\right)
$$

(iii) Le cas où $X \in T_{3, \infty}^{H} W, Y \in T_{1}^{H} W$. - Soit $U$ (resp. $Z$ ) une $\operatorname{section} \mathcal{C}^{\infty}$ de $T S$ (resp. $T Y$ ); alors

$$
\begin{array}{r}
T_{3, \infty}\left(U_{3, \infty}^{H}, Z_{1}^{H}\right)=\nabla_{U_{3, \infty}^{H}}^{T^{H}} Z_{1}^{H}+A_{3, \infty}\left(U_{3, \infty}^{H}\right) Z_{1}^{H}-\left[U_{3, \infty}^{H}, Z_{1}^{H}\right] \\
=-\left[U_{3, \infty}^{H}, Z_{1}^{H}\right]+\left[\nabla_{U_{2}^{H}}^{T Y} Z\right]_{1}^{H}+A_{3, \infty}\left(U_{3, \infty}^{H}\right) Z_{1}^{H} \\
=T_{1}\left(U_{3, \infty}^{H}, Z_{1}^{H}\right)+\left[T_{2}\left(U_{2}^{H}, Z\right)\right]_{1}^{H}+A_{3, \infty}\left(U_{3, \infty}^{H}\right) Z_{1}^{H} .
\end{array}
$$

(iv) Le cas où $X, Y \in T Z$. - Soient $X, Y$ des sections $\mathcal{C}^{\infty}$ de $T Z$. D'après [B4, th. 1.1] et (1.13), on a

$$
\begin{aligned}
T_{3, \infty}(X, Y) & =\nabla_{\infty, X}^{T Z} Y-\nabla_{\infty, Y}^{T Z} X-[X, Y] \\
& =\lim _{T \rightarrow \infty}\left(\nabla_{T, X}^{T Z} Y-\nabla_{T, Y}^{T Z} X-[X, Y]\right)=0 .
\end{aligned}
$$

Nous avons bien démontré la proposition 1.5 .

ThÉORÈme 1.6. - Quand $T \rightarrow+\infty$,

$$
T_{3, T}=T_{3, \infty}+O\left(\frac{1}{T^{2}}\right) .
$$

Preuve. - On a des identifications de fibrés vectoriels $\mathcal{C}^{\infty}$ sur $V$

$$
T W=T_{3, T}^{H} W \oplus T Z, \quad T_{3, T}^{H} W \simeq \pi_{3}^{*} T S .
$$

Soit $\nabla^{T_{3, T}^{H} W}$ la connexion induite par $\nabla^{T S}$ sur $T_{3, T}^{H} W$. Alors $T_{3, T}$ est la torsion de la connexion $\nabla^{T_{3, T}^{H} W} \oplus \nabla_{T}^{T Z}$. En utilisant la proposition 1.2, (1.13) et (1.18), on a $(1.24)$. 
THÉORÈME 1.7.

(i) Pour $X \in T X, Z \in T Z, U, U^{\prime} \in T S$, on pose

$$
Y_{T}=Z+U_{3, T}^{H}, \quad Y_{T}^{\prime}=U_{3, T}^{\prime H} .
$$

Alors quand $T \rightarrow+\infty$, on $a:$

$$
\begin{aligned}
T^{2}\left\langle S_{3, T}(X) Y_{T}, Y_{T}^{\prime}\right\rangle_{T}=\left\langle S_{1}(\right. & \left.X) Y_{\infty}, Y_{\infty}^{\prime}\right\rangle \\
& +\frac{1}{2} L_{X}\left\langle h^{\prime} Y_{\infty}^{\prime}, P^{T^{H} Z} Z\right\rangle_{\pi_{1}^{*} g^{T Y}}+O\left(\frac{1}{T^{2}}\right)
\end{aligned}
$$

(ii) Pour $X_{1}, Y_{1} \in T Y, U, U^{\prime} \in T S$, on pose

$$
X=X_{1,1}^{H}, \quad Y_{T}=Y_{1,1}^{H}+U_{3, T}^{H}, \quad Y_{T}^{\prime}=U_{3, T}^{\prime H} .
$$

Alors quand $T \rightarrow+\infty$, on $a:$

$$
\left\langle S_{3, T}(X) Y_{T}, Y_{T}^{\prime}\right\rangle_{T}=\left\langle S_{2}\left(X_{1}\right)\left(Y_{1}+U_{2}^{H}\right), U_{2}^{\prime H}\right\rangle+O\left(\frac{1}{T^{2}}\right) .
$$

Preuve. - (i) D'après [B4, (1.5), (1.6)], (1.8) et le théorème 1.6, quand $T \rightarrow+\infty$, on a :

$$
\begin{aligned}
T^{2}\left\langle S_{3, T}(X) Y_{T}, Y_{T}^{\prime}\right\rangle_{T}=- & T^{2}\left\langle T_{3, T}\left(U_{3, T}^{\prime H}, Z\right), X\right\rangle_{g_{T}^{T Z}} \\
& +\frac{1}{2} T^{2}\left\langle T_{3, T}\left(U_{3, T}^{H}, U_{3, T}^{\prime H}\right), X\right\rangle_{g_{T}^{T Z}} \\
=- & \left\langle P^{T X} T_{3, \infty}\left(U_{3, \infty}^{\prime H}, Z\right), X\right\rangle_{g^{T X}} \\
& +\frac{1}{2}\left\langle P^{T X} T_{3, \infty}\left(U_{3, \infty}^{H}, U_{3, \infty}^{\prime H}\right), X\right\rangle_{g^{T X}}+O\left(\frac{1}{T^{2}}\right) .
\end{aligned}
$$

Par [B4, (1.5), (1.6)], (1.11), (1.19), on a :

$$
\begin{aligned}
&(1.29) \quad T^{2}\left\langle S_{3, T}(X) Y_{T}, Y_{T}^{\prime}\right\rangle_{T} \\
&=-\left\langle T_{1}\left(U_{3, \infty}^{\prime H}, P^{T X} Z\right), X\right\rangle_{g^{T X}} \\
&+\frac{1}{2}\left\langle T_{1}\left(U_{3, \infty}^{H}+P^{T^{H}} Z Z_{3, \infty}\right), X\right\rangle_{g^{T X}} \\
& \quad+\frac{1}{2}\left\langle\left[X, h^{\prime} U_{3, \infty}^{\prime H}\right], P^{T^{H} Z} Z\right\rangle_{\pi_{1}^{*} g^{T Y}}+O\left(\frac{1}{T^{2}}\right) \\
&=\left\langle S_{1}(X) Y_{\infty}, Y_{\infty}^{\prime}\right\rangle+\frac{1}{2} L_{X}\left\langle h^{\prime} U_{3, \infty}^{\prime H}, P^{T^{H}} Z Z\right\rangle_{\pi_{1}^{*} g^{T Y}}+O\left(\frac{1}{T^{2}}\right) .
\end{aligned}
$$

TOME $127-1999-\mathrm{N}^{\circ} 4$ 
(ii) D'après $[\mathrm{B} 4,(1.5),(1.6)],(1.8)$ et le théorème 1.6 , quand $T \rightarrow+\infty$, on a :

$$
\begin{aligned}
& \left\langle S_{3, T}(X) Y_{T}, Y_{T}^{\prime}\right\rangle \\
& =-\left\langle T_{3, T}\left(U_{3, T}^{\prime H}, Y_{1,1}^{H}\right), X_{1,1}^{H}\right\rangle_{g_{T}^{T Z}} \\
& \quad+\frac{1}{2}\left\langle T_{3, T}\left(U_{3, T}^{H}, U_{3, T}^{\prime H}\right), X_{1,1}^{H}\right\rangle_{g_{T}^{T Z}} \\
& =-\left\langle P^{T^{H}} Z_{T_{3, \infty}}\left(U_{3, \infty}^{\prime H}, Y_{1,1}^{H}\right), X_{1,1}^{H}\right\rangle_{\pi_{1}^{*} g^{T Y}} \\
& \quad+\frac{1}{2}\left\langle P^{T^{H} Z} T_{3, \infty}\left(U_{3, \infty}^{H}, U_{3, \infty}^{\prime H}\right), X_{1,1}^{H}\right\rangle_{\pi_{1}^{*} g^{T Y}}+O\left(\frac{1}{T^{2}}\right) \\
& =-\left\langle T_{2}\left(U_{2}^{\prime H}, Y_{1}\right), X_{1}\right\rangle_{g^{T Y}}+\frac{1}{2}\left\langle T_{2}\left(U_{2}^{H}, U_{2}^{\prime H}\right), X_{1}\right\rangle_{g^{T Y}}+O\left(\frac{1}{T^{2}}\right) \\
& =\left\langle S_{2}\left(X_{1}\right)\left(Y_{1}+U_{2}^{H}\right), U_{2}^{\prime H}\right\rangle+O\left(\frac{1}{T^{2}}\right) .
\end{aligned}
$$

On a fini la preuve du théorème 1.7 .

b) L'asymptotique des tenseurs associés aux fibrations kählériennes.

Soient $W, V, S$ des variétés complexes. Soient $\pi_{1}: W \rightarrow V, \pi_{2}: V \rightarrow S$ des submersions holomorphes de fibres compactes $X, Y$. Alors $\pi_{3}=\pi_{2} \circ \pi_{1}: W \rightarrow S$ est une submersion holomophe de fibre compacte $Z$. Soient $T X, T Y, T Z$ les fibrés tangents holomorphes relatifs.

Soit $\omega^{V}$ (resp. $\widetilde{\omega}^{W}$ ) une $(1,1)$-forme réelle, fermée, $\mathcal{C}^{\infty}$ sur $V$ (resp. $W$ ) dont la restriction à chaque fibre $Y$ (resp. $Z$ ) définit une métrique hermitienne $g^{T Y}$ (resp. $\tilde{g}^{T Z}$ ) sur le fibré tangent relatif $T Y$ (resp. $T Z$ ).

Dans la suite, on utilisera un indice $\mathbb{R}$ pour distinguer les fibrés réels des fibrés complexes.

Pour $T>0$, on pose

$$
\omega_{T}^{W}=\frac{1}{T^{2}} \widetilde{\omega}^{W}+\pi_{1}^{*} \omega^{V}, \quad \omega^{W}=\omega_{1}^{W}
$$

Soient $g^{T X}$ et $g_{T}^{T Z}$ les métriques hermitiennes sur $T X, T Z$ induites par $\widetilde{\omega}^{W}$ et $\omega_{T}^{W}$. Soient $\nabla^{T X}, \nabla^{T Y}, \nabla_{T}^{T Z}$ les connexions holomorphes hermitiennes sur $\left(T X, g^{T X}\right),\left(T Y, g^{T Y}\right),\left(T Z, g_{T}^{T Z}\right)$. Soient $T_{1}^{H} W, T_{2}^{H} V, T_{3, T}^{H} W$ les sous-fibrés vectoriels de $T W, T V, T W$ orthogonaux à $\overline{T X}, \overline{T Y}, \overline{T Z}$ pour $\widetilde{\omega}^{W}, \omega^{V}, \omega_{T}^{W}$ [B4, th. 2.2].

Par [BGS2, th. 1.7], la connexion $\nabla^{T_{\mathbb{R}}} X$ sur $T_{\mathbb{R}} X$ préserve la structure complexe de $T_{\mathbb{R}} X$, et elle induit la connexion holomorphe hermitienne $\nabla^{T X}$ sur $T X$ pour $g^{T X}$. 
Définition 1.8. - Soient $h \in \operatorname{End}\left(T^{H} Z\right), h^{\prime} \in \operatorname{Hom}\left(T_{3, \infty}^{H} W, T^{H} Z\right)$ tels que si $U, V \in T Y, X \in T_{2}^{H} V$,

$$
\left\{\begin{array}{l}
\left\langle U_{1}^{H}, \bar{V}_{1}^{H}\right\rangle_{\tilde{g}^{T Z}}=\left\langle h U_{1}^{H}, \bar{V}_{1}^{H}\right\rangle_{g^{T^{H} Z}}, \\
i \widetilde{\omega}^{W}\left(X_{1}^{H}, \bar{V}_{1}^{H}\right)=\left\langle h^{\prime} X_{1}^{H}, \bar{V}_{1}^{H}\right\rangle_{g^{T^{H} Z}} .
\end{array}\right.
$$

Alors $h$ est une section auto-adjointe définie positive de $\operatorname{End}\left(T^{H} Z\right)$. Soit $\bar{h} \in$ $\operatorname{End}\left(\overline{T^{H} Z}\right)\left(\right.$ resp. $\left.\bar{h}^{\prime} \in \operatorname{Hom}\left(\overline{T_{3, \infty}^{H} W}, \overline{T^{H} Z}\right)\right)$ le conjugué de $h$ (resp. $\left.h^{\prime}\right)$. On prolonge $h$ à $T_{\mathbb{R}}^{H} Z$ (resp. $h^{\prime}$ de $T_{3, \infty, \mathbb{R}}^{H} W$ à $\left.T_{\mathbb{R}}^{H} Z\right)$.

Notons que $\widetilde{\omega}^{W}, \omega^{V}$ ne définissent en général pas des métriques kählériennes sur $W, V$. Toutefois, si $s_{0} \in S$, si $\omega^{S}$ est une forme de Kähler sur un voisinage $U$ de $s_{0}$ dans $S$, pour $\lambda \gg 0, \widetilde{\omega}^{W}+\lambda \pi_{3}^{*} \omega^{S}, \omega^{V}+\lambda \pi_{2}^{*} \omega^{S}$ sont des formes kählériennes sur $\pi_{3}^{-1}(U), \pi_{2}^{-1}(U)$. On peut alors effectuer les constructions du $\S 1 \mathrm{a}$ ) sur $\pi_{3}^{-1}(U), \pi_{2}^{-1}(U)$. Les constructions qui suivent la définition 1.1 montrent que les objets construits ne dépendent pas de $\omega^{S}$, et sont donc globalement définis sur $W$ ou sur $V$.

Comme complément, on donne l'asymptotique de $\nabla_{T}^{T Z}$.

On rappelle qu'on a l'identification de fibrés vectoriels $\mathcal{C}^{\infty}$ sur $W$

$$
T Z \simeq T^{H} Z \oplus T X
$$

Soit $\nabla^{T^{H} Z}$ la connexion induite par $\nabla^{T Y}$, soit ${ }^{0} \nabla^{T Z}$ la connexion sur $T Z$

$$
{ }^{0} \nabla^{T Z}=\nabla^{T^{H} Z} \oplus \nabla^{T X} \text {. }
$$

Soit $A \in T^{*(0,1)} W \otimes \operatorname{Hom}\left(T^{H} Z, T X\right)$ tel que $\nabla^{T Z^{\prime \prime}}={ }^{0} \nabla^{T Z^{\prime \prime}}+A$. Soit $A^{*}$ l'adjoint de $A$ associé aux métriques $g^{T^{H} Z}$ et $g^{T X}$.

On écrit $\nabla_{T}^{T Z}$ sous forme matricielle relativement au scindage $T Z \simeq T^{H} Z \oplus$ $T X$. D'après [BerB, (7.24)], on a

$$
\nabla_{T}^{T Z}=\left[\begin{array}{cc}
\nabla^{T^{H} Z}+\left(T^{2}+h\right)^{-1} \nabla^{T^{H} Z^{\prime}} h & -\left(T^{2}+h\right)^{-1} A^{*} \\
A & \nabla^{T X}
\end{array}\right] .
$$

On pose

$$
\nabla_{\infty}^{T Z}=\left[\begin{array}{cc}
\nabla^{T^{H} Z} & 0 \\
A & \nabla^{T X}
\end{array}\right]
$$

D'après (1.35), on a :

ThÉORÈme 1.9. - Quand $T \rightarrow+\infty$, on $a:$

$$
\nabla_{T}^{T Z}=\nabla_{\infty}^{T Z}+O\left(\frac{1}{T^{2}}\right) .
$$




\section{Formes de torsion analytique}

Dans ce paragraphe, pour fixer les notations, on rappelle la construction de formes de torsion analytique dans [BKö] associées aux fibrations kählériennes $\pi: W \rightarrow V$. Pour une description plus complète, on se réfère à $[\mathrm{B} 4, \S 2]$.

Ce paragraphe est organisé de la façon suivante. Dans a), on rappelle la construction de la superconnexion de Levi-Civita associée à une fibration [B1] Dans b), on construit des formes de torsion analytique de [BKö].

\section{a) Superconnexion de Levi-Civita d'une fibration kählérienne.}

Soient $W$ et $V$ des variétés complexes, soit $\pi: W \rightarrow V$ une submersion holomorphe de fibre compacte $X$. Soit $\omega^{W}$ une $(1,1)$-forme réelle, fermée, $\mathcal{C}^{\infty}$ sur $W$ qui induit une métrique hermitienne $g^{T X}$ sur $T X$.

Soit $T^{H} W$ le sous-fibré de $T W$ orthogonal à $\overline{T X}$ pour $\omega^{W}$. Pour $U \in T V$, soit $U^{H} \in T^{H} W$ le relèvement de $U$ dans $T^{H} W$ qui est tel que $\pi_{*} U^{H}=U$.

Soit $\xi$ un fibré vectoriel holomorphe sur $W$. Soit $h^{\xi}$ une métrique hermitienne sur $\xi$. Soient $\nabla^{T X}, \nabla^{\xi}$ les connexions holomorphes hermitiennes sur $\left(T X, g^{T X}\right)$, $\left(\xi, h^{\xi}\right)$. Soit $\nabla^{\Lambda\left(T^{*(0,1)} X\right)}$ la connexion sur $\Lambda\left(T^{*(0,1)} X\right)$ induite par $\nabla^{T X}$. Soit $\nabla^{\Lambda\left(T^{*(0,1)} X\right) \otimes \xi}$ la connexion sur $\Lambda\left(T^{*(0,1)} X\right) \otimes \xi$

$$
\nabla^{\Lambda\left(T^{*(0,1)} X\right) \otimes \xi}=\nabla^{\Lambda\left(T^{*(0,1)} X\right)} \otimes 1+1 \otimes \nabla^{\xi}
$$

Pour $b \in V$, soit $\left(\Omega\left(X_{b}, \xi_{\mid X_{b}}\right), \bar{\partial}^{X_{b}}\right)$ le complexe de Dolbeault relatif des sections $\mathcal{C}^{\infty}$ de $\left(\Lambda\left(T^{*(0,1)} X\right) \otimes \xi\right)_{\mid X_{b}}$. Alors $\Omega\left(X_{b}, \xi_{\mid X_{b}}\right)$ va être considéré comme une fibre d'un fibré vectoriel de dimension infinie sur $V$ dont les sections $\mathcal{C}^{\infty}$ sont identifiées aux sections $\mathcal{C}^{\infty}$ de $\Lambda\left(T^{*(0,1)} X\right) \otimes \xi$ sur $W$.

Soit $*^{T X}$ l'opérateur de Hodge associé à $g^{T X}$ sur $\Lambda\left(T_{\mathbb{R}}^{*} X\right)$. On définit le produit hermitien sur $\Omega\left(X_{b}, \xi_{\mid X_{b}}\right)$,

$$
\alpha, \alpha^{\prime} \in \Omega\left(X_{b}, \xi_{\mid X_{b}}\right) \longmapsto\left\langle\alpha, \alpha^{\prime}\right\rangle_{b}=\frac{1}{(2 \pi)^{\operatorname{dim} X}} \int_{X_{b}}\left\langle\alpha \wedge *^{T X} \alpha^{\prime}\right\rangle_{h^{\xi}}
$$

Soit $\mathrm{d} v_{X}$ la forme de volume sur $X$ associée à $g^{T X}$ sur $T X$. Soit \langle\rangle$_{\Lambda\left(T^{*(0,1)} X\right) \otimes \xi}$ le produit hermitien sur $\Lambda\left(T^{*(0,1)} X\right) \otimes \xi$ associé aux métriques $g^{T X}, h^{\xi}$ sur $T X, \xi$. Alors pour $\alpha, \alpha^{\prime} \in \Omega\left(X_{b}, \xi_{\mid X_{b}}\right)$, on a :

$$
\left\langle s, s^{\prime}\right\rangle_{b}=(2 \pi)^{-\operatorname{dim} X} \int_{X_{b}}\left\langle s, s^{\prime}\right\rangle_{\Lambda\left(T^{*(0,1)} X\right) \otimes \xi} \mathrm{d} v_{X}
$$

Soit $\bar{\partial}^{X_{b} *}$ l'adjoint formel de $\bar{\partial}^{X_{b}}$ pour le produit hermitien (2.2). 
DÉfinition 2.1. - Si s est une section $\mathcal{C}^{\infty}$ de $\Omega\left(X, \xi_{\mid X}\right)$, si $U \in T_{\mathbb{R}} V$, on pose :

$$
\nabla_{U}^{\Omega\left(X, \xi_{\mid X}\right)} s=\nabla_{U^{H}}^{\Lambda\left(T^{*(0,1)} X\right) \otimes \xi} s
$$

$\operatorname{Par}[\mathrm{B} 1, \S 1(\mathrm{~b})], \nabla^{\Omega\left(X, \xi_{\mid X}\right)}$ est une connexion sur le fibré vectoriel $\Omega\left(X, \xi_{\mid X}\right)$. Soient $\nabla^{\Omega\left(X, \xi_{\mid X}\right)^{\prime}}, \nabla^{\Omega\left(X, \xi_{\mid X}\right)^{\prime \prime}}$ les parties holomorphes et antiholomorphes de $\nabla^{\Omega\left(X, \xi_{\mid X}\right)}$.

La fibre $\Lambda\left(T^{*(0,1)} X\right) \otimes \xi$ est un $c\left(T_{\mathbb{R}} X\right)$-module de Clifford. En effet, si $U \in T X$, soit $U^{\prime} \in T^{*(0,1)} X$ qui correspond à $U$ par la métrique $g^{T X}$. Si $U, V \in T X$, on pose :

$$
c(U)=\sqrt{2} U^{\prime} \wedge, \quad c(\bar{V})=-\sqrt{2} i_{\bar{V}}
$$

Pour $X \in T_{\mathbb{R}} X \otimes_{\mathbb{R}} \mathbb{C}=T X \oplus \overline{T X}$, on définit $c(X)$ par prolongement $\mathbb{C}$-linéaire.

Soit $f_{1}, \ldots, f_{2 m}$ une base de $T_{\mathbb{R}} V$ et soit $f^{1}, \ldots, f^{2 m}$ la base duale de $T_{\mathbb{R}}^{*} V$.

DÉfinition 2.2. - Soit

$$
c(T)=\frac{1}{2} \sum f^{\alpha} f^{\beta} c\left(T\left(f_{\alpha}^{H}, f_{\beta}^{H}\right)\right) .
$$

Alors $c(T)$ est une section de $\left(\Lambda\left(T_{\mathbb{R}}^{*} V\right) \widehat{\otimes} \operatorname{End}\left(\Lambda\left(T^{*(0,1)} X\right) \otimes \xi\right)\right)^{\text {impair }}$. On définit aussi $c\left(T^{1,0}\right), c\left(T^{0,1}\right)$ par des formules semblables à $(2.5)$, de sorte que

$$
c(T)=c\left(T^{1,0}\right)+c\left(T^{0,1}\right) .
$$

DÉfINITION 2.3. - Pour $u>0$, on pose :

$$
\left\{\begin{array}{l}
B_{u}^{\prime \prime}=\nabla^{\Omega\left(X, \xi_{\mid X}\right)^{\prime \prime}}+\sqrt{u} \bar{\partial}^{X}-\frac{c\left(T^{1,0}\right)}{2 \sqrt{2} u} \\
B_{u}^{\prime}=\nabla^{\Omega\left(X, \xi_{\mid X}\right)^{\prime}}+\sqrt{u} \bar{\partial}^{X *}-\frac{c\left(T^{0,1}\right)}{2 \sqrt{2} u} \\
B_{u}=B_{u}^{\prime}+B_{u}^{\prime \prime} .
\end{array}\right.
$$

D'après [BGS2, $\S 2(\mathrm{a})], B_{u}$ est la superconnexion de Levi-Civita $A_{u / 2}$ définie $\operatorname{par}[\mathrm{B} 1, \S 3]$.

Soit $N_{X}$ l'opérateur de nombre de $\Lambda\left(T^{*(0,1)} X\right) \otimes \xi$, qui agit par multiplication par $k$ sur $\Lambda^{k}\left(T^{*(0,1)} X\right) \otimes \xi$. Alors l'opérateur $N_{X}$ définit une $\mathbb{Z}$-graduation $\operatorname{sur} \Lambda\left(T^{*(0,1)} X\right) \otimes \xi$.

томе $127-1999-\mathrm{N}^{\circ} 4$ 
Pour tout $U, U^{\prime} \in T_{\mathbb{R}} V$, on pose :

$$
\omega^{H \bar{H}}\left(U, U^{\prime}\right)=\omega^{W}\left(U^{H}, U^{\prime H}\right) .
$$

DÉfinition 2.4. - Pour $u>0$, on pose :

$$
N_{u}=N_{X}+i \frac{\omega^{H \bar{H}}}{u} .
$$

b) Formes de torsions analytiques.

Soit $R^{\bullet} \pi_{*} \xi=\bigoplus_{k=0}^{\operatorname{dim} X} R^{k} \pi_{*} \xi$ l'image directe de $\xi$ par $\pi$. On suppose que, pour tout $k, 0 \leq k \leq \operatorname{dim} X, R^{k} \pi_{*} \xi$ est localement libre. Pour $b \in V$, soit $H\left(X_{b}, \xi_{\mid X_{b}}\right)$ la cohomologie du faisceau des sections holomorphes de $\xi_{\mid X_{b}}$ sur $X_{b}$. Alors les $H\left(X_{b}, \xi_{\mid X_{b}}\right)$ sont les fibres du fibré holomorphe $\mathbb{Z}$-gradué $H\left(X, \xi_{\mid X}\right)$ sur $V$; plus précisément,

$$
R^{\bullet} \pi_{*} \xi=H\left(X, \xi_{\mid X}\right)
$$

Désormais, on suppose que $R^{k} \pi_{*} \xi,(0 \leq k \leq \operatorname{dim} X)$ est localement libre, et on identifie $R^{\bullet} \pi_{*} \xi$ à $H\left(X, \xi_{\mid X}\right)$.

On pose

$$
D_{b}^{X}=\bar{\partial}^{X_{b}}+\bar{\partial}^{X_{b} *}, \quad K\left(X_{b}, \xi_{\mid X_{b}}\right)=\operatorname{ker} D_{b}^{X} .
$$

Par la théorie de Hodge

$$
H\left(X_{b}, \xi_{\mid X_{b}}\right) \simeq K\left(X_{b}, \xi_{\mid X_{b}}\right) .
$$

Donc $K\left(X_{b}, \xi_{\mid X_{b}}\right)$ est de dimension localement constante. Les $K\left(X_{b}, \xi_{\mid X_{b}}\right)$ sont les fibres d'un fibré $\mathcal{C}^{\infty}, K\left(X, \xi_{\mid X}\right)$, sur $V$. D'après [BGS3, th. 3.5], l'isomorphisme canonique des fibres $(2.12)$ provient d'un isomorphisme de fibrés vectoriels $\mathbb{Z}$-gradués sur $V$,

$$
R^{\bullet} \pi_{*} \xi \simeq K\left(X, \xi_{\mid X}\right) .
$$

De plus $K\left(X, \xi_{\mid X}\right)$ hérite du produit hermitien $(2.2)$ sur $\Omega\left(X, \xi_{\mid X}\right)$. Soit $h^{R \pi_{*} \xi}$ la métrique correspondante sur $R^{\bullet} \pi_{*} \xi \operatorname{par}(2.13)$. Pour $0 \leq k \leq \operatorname{dim} X$, $\left(R^{k} \pi_{*} \xi, h^{R^{k} \pi_{*} \xi}\right)$ est un fibré vectoriel holomorphe hermitien sur $V$.

Définition 2.5. - Soit $P^{W}$ l'espace vectoriel de formes réelles sur $W$ qui sont la somme de formes $\mathcal{C}^{\infty}$ de type $(p, p)$. Soit

$$
\begin{aligned}
& P^{W, 0}=\left\{\alpha \in P^{W}, \text { il existe des formes } \beta, \gamma \mathcal{C}^{\infty} \text { sur } W\right. \\
&\text { telles que } \alpha=\partial \beta+\bar{\partial} \gamma\} .
\end{aligned}
$$

On définit de la même manière $P^{V}$ et $P^{V, 0}$. 
Soit $P$ une série formelle ad-invariante définie sur les matrices carrées. Soit $\left(E, g^{E}\right)$ un fibré vectoriel holomorphe hermitien sur $W$. Soit $\nabla^{E}$ la connexion holomorphe hermitienne sur $E$, et soit $R^{E}$ sa courbure. Soit

$$
P\left(E, g^{E}\right)=P\left(\frac{-R^{E}}{2 \pi i}\right)
$$

Alors $P\left(E, g^{E}\right)$ est une forme fermée dans $P^{W}$ dont la classe de cohomologie $P(E)$ ne dépend pas de $g^{E}$.

On rappelle que, si $A$ est une matrice carrée, alors

$$
\operatorname{Td}(A)=\operatorname{det}\left(\frac{A}{1-\mathrm{e}^{-A}}\right), \quad \operatorname{ch}(A)=\operatorname{Tr}[\exp (A)]
$$

On pose

$$
\operatorname{Td}^{\prime}(A)=\frac{\partial}{\partial b} \operatorname{Td}(A+b I)_{\mid b=0}
$$

Soit

$$
\operatorname{ch}\left(R^{\bullet} \pi_{*} \xi, h^{R \pi_{*} \xi}\right)=\sum_{k=0}^{\operatorname{dim} X}(-1)^{k} \operatorname{ch}\left(R^{k} \pi_{*} \xi, h^{R^{k} \pi_{*} \xi}\right)
$$

Maintenant, on définit les formes de torsion analytique $T\left(\omega^{W}, h^{\xi}\right)$, en utilisant la superconnexion de Levi-Civita $B_{u}$ comme en [BKö, déf. 3.8]. Par [BKö, th. 3.9], la forme $T\left(\omega^{W}, h^{\xi}\right)$ est dans $P^{V}$, et

$$
\frac{\bar{\partial} \partial}{2 \pi i} T\left(\omega^{W}, h^{\xi}\right)=\operatorname{ch}\left(R^{\bullet} \pi_{*} \xi, h^{R \pi_{*} \xi}\right)-\int_{X} \operatorname{Td}\left(T X, g^{T X}\right) \operatorname{ch}\left(\xi, h^{\xi}\right)
$$

Dans [BKö, th. 3.10], ils ont établi des formules d'anomalie pour ces formes.

\section{Fonctorialité des formes de torsion analytique}

Le but principal de cet article est d'établir les théorèmes 3.5 et 3.11.

Ce paragraphe est organisé de la façon suivante. Dans a), on donne les hypothèses et les notations de cet article. Dans b), on énonce le résultat dans le cas où $\xi$ est $\pi_{1 *}$ et $\pi_{3 *}$ acyclique. Dans $\mathrm{c}$ ), on énonce le résultat dans le cas où $\pi_{1}$ et $V$ sont projectives.

On utilise les mêmes notations qu'au $\S 1 \mathrm{~b}$ ).

$$
\text { TOME } 127-1999-\mathrm{N}^{\circ} 4
$$




\section{a) Hypothèses et notations.}

Soient $W, V, S$ des variétés complexes. Soient $\pi_{1}: W \rightarrow V, \pi_{2}: V \rightarrow S$ des submersions holomorphes de fibres compactes $X, Y$. Alors $\pi_{3}=\pi_{2} \circ \pi_{1}: W \rightarrow S$ est une submersion holomorphe de fibre compacte $Z$. Soit $\xi$ un fibré vectoriel holomorphe sur $W$.

Soit $\omega^{W}$ (resp. $\omega^{V}$ ) une $(1,1)$-forme réelle, fermée, $\mathcal{C}^{\infty}$ sur $W$ (resp. $V$ ) dont la restriction à chaque fibre $Z$ (resp. $Y$ ) définit une métrique hermitienne $g^{T Z}$ (resp. $g^{T Y}$ ) sur $T Z$ (resp. $T Y$ ). Soit $h^{\xi}$ une métrique hermitienne sur $\xi$. On rappelle que $g^{T X}$ est la métrique sur $T X$ induite par $\omega^{W}$.

On suppose que $R^{\bullet} \pi_{1 *} \xi, R^{\bullet} \pi_{3 *} \xi$ et $R^{\bullet} \pi_{2 *} R^{\bullet} \pi_{1 *} \xi$ sont localement libres. Comme au $\S 2 \mathrm{~b}$ ), on peut identifier le fibré $R^{\bullet} \pi_{1 *} \xi$ (resp. $R^{\bullet} \pi_{3 *} \xi$, resp. $\left.R^{\bullet} \pi_{2 *} R^{\bullet} \pi_{1 *} \xi\right)$ aux éléments harmoniques associés dans le complexe de Dolbeault relatif $\Omega\left(X, \xi_{\mid X}\right)$ (resp. $\Omega\left(Z, \xi_{\mid Z}\right)$, resp. $\Omega\left(Y, R^{\bullet} \pi_{1 *} \xi_{\mid Y}\right)$ ). Soient $h^{R \pi_{1 *} \xi}, h^{R \pi_{3 *} \xi}$, $h^{R \pi_{2 *} R \pi_{1 *} \xi}$ les métriques associées définies au $\S 2 \mathrm{~b}$ ).

Sur $W$, on a une suite exacte de fibrés vectoriels holomorphes hermitiens :

$$
0 \rightarrow T X \longrightarrow T Z \longrightarrow \pi_{1}^{*} T Y \rightarrow 0
$$

Par une construction de [BGS1, $\S 1 \mathrm{f})$ ], il existe une unique classe de Bott-Chern $\widetilde{\mathrm{Td}}\left(T Z, T Y, g^{T Z}, g^{T Y}\right) \in P^{W} / P^{W, 0}$ telle que

$$
\begin{aligned}
\frac{\bar{\partial} \partial}{2 \pi i} \widetilde{\mathrm{Td}}\left(T Z, T Y, g^{T Z}\right. & \left., g^{T Y}\right) \\
& =\operatorname{Td}\left(T Z, g^{T Z}\right)-\pi_{1}^{*}\left(\operatorname{Td}\left(T Y, g^{T Y}\right)\right) \operatorname{Td}\left(T X, g^{T X}\right) .
\end{aligned}
$$

Soient $T_{1}\left(\omega^{W}, h^{\xi}\right), T_{2}\left(\omega^{V}, h^{R \pi_{1 *} \xi}\right), T_{3}\left(\omega^{W}, h^{\xi}\right)$ les formes de torsion analytique sur $V, S, S$ pour $\pi_{1}, \pi_{2}, \pi_{3}$ définies au $\S 2 \mathrm{~b}$ ).

Soit $s \in S$; alors d'après [Grot, $\S 3.7$ ] (ou voir le $\S 10 \mathrm{~b}$ )), il existe une suite spectrale de Leray pour le foncteur dérivé du composé $\pi_{3 *}=\pi_{2 *} \circ \pi_{1 *}$. On le notera $\left(E_{r, s}, \mathrm{~d}_{r, s}\right)$. On a :

$$
\left\{\begin{array}{l}
E_{2}^{i, j}=H^{i}\left(Y, R^{j} \pi_{1 *} \xi_{\mid Y}\right) \simeq R^{i} \pi_{2 *} R^{j} \pi_{1 *} \xi \\
E_{r} \Longrightarrow H\left(Z, \xi_{\mid Z}\right) \simeq R^{\bullet} \pi_{3 *} \xi
\end{array}\right.
$$

Donc $E_{2}^{\bullet, \bullet}$ est un fibré vectoriel holomorphe sur $S$. Soit $h^{E_{2}}$ la métrique sur $E_{2}$ induite par $h^{R \pi_{2 *} R \pi_{1 *} \xi}$. Soit $h^{H\left(Z, \xi_{\mid Z}\right)}$ la métrique sur $H\left(Z, \xi_{\mid Z}\right)$ définie au $\left.\S 2 \mathrm{~b}\right)$ associée à $g^{T Z}, h^{\xi}$.

b) Le cas acyclique.

Dans cette partie, on suppose que pour $i>0$, on a

$$
R^{i} \pi_{1 *} \xi=0, \quad R^{i} \pi_{3 *} \xi=0 .
$$

BULLETIN DE LA SOCIÉTÉ MATHÉMATIQUE DE FRANCE 
Alors la suite spectrale de Leray pour chaque fibre $Z_{s}(s \in S)$ dégénère à partir de $E_{2}$, et de plus,

$$
E_{2}=H\left(Z, \xi_{\mid Z}\right)=H^{0}\left(Z, \xi_{\mid Z}\right)
$$

Donc par la construction de [BGS1, 1 f)], la classe de Bott-Chern c $\widetilde{c h}\left(E_{2}\right.$, $\left.H\left(Z, \xi_{\mid Z}\right), h^{E_{2}}, h^{H\left(Z, \xi_{\mid Z}\right)}\right) \in P^{S} / P^{S, 0}$ est bien définie.

DÉfinition 3.1. - Soit $\Delta\left(\omega^{W}, \omega^{V}, h^{\xi}\right)$ la forme dans $P^{S} / P^{S, 0}$

$$
\begin{aligned}
& \Delta\left(\omega^{W}, \omega^{V}, h^{\xi}\right)=T_{3}\left(\omega^{W}, h^{\xi}\right)-T_{2}\left(\omega^{V}, h^{R \pi_{1 *} \xi}\right)-\int_{Y} \operatorname{Td}\left(T Y, g^{T Y}\right) T_{1}\left(\omega^{W}, h^{\xi}\right) \\
& -\widetilde{\operatorname{ch}}\left(E_{2}, H\left(Z, \xi_{\mid Z}\right), h^{E_{2}}, h^{H\left(Z, \xi_{\mid Z}\right)}\right)+\int_{Z} \widetilde{\operatorname{Td}}\left(T Z, T Y, g^{T Z}, g^{T Y}\right) \operatorname{ch}\left(\xi, h^{\xi}\right) .
\end{aligned}
$$

Proposition 3.2. - On a

$$
\frac{\bar{\partial} \partial}{2 \pi i} \Delta\left(\omega^{W}, \omega^{V}, h^{\xi}\right)=0
$$

Preuve. - En utilisant (3.2) et (2.18), on a (3.7).

Remarque 3.3. - Soit $\omega^{\prime W}$ (resp. $\omega^{\prime V}$ ) une autre (1,1)-forme réelle, fermée, $\mathcal{C}^{\infty}$ sur $W$ (resp. $V$ ) qui induit une métrique hermitienne sur $T Z$ (resp. $T Y$ ). Soit $h^{\prime \xi}$ une autre métrique hermitienne sur $\xi$. Alors, en utilisant [BKö, th. 3.10], on peut vérifier que, comme dans [BerB, $\S 3(\mathrm{~b})]$, on a

$$
\Delta\left(\omega^{W}, \omega^{V}, h^{\xi}\right)=\Delta\left(\omega^{\prime W}, \omega^{\prime V}, h^{\prime \xi}\right) \quad \text { dans } \quad P^{S} / P^{S, 0} .
$$

Remarque 3.4. - Soit $\xi^{\prime}$ un fibré vectoriel holomorphe sur $S$. Si on suppose que $S$ est kählérienne et compacte, en utilisant [BerB, th. 3.1], [BGS3, th. 1.23], on peut vérifier aussi que

$$
\int_{S} \operatorname{Td}(T S) \operatorname{ch}\left(\xi^{\prime}\right) \Delta\left(\omega^{W}, \omega^{V}, h^{\xi}\right)=0 .
$$

Le but de cet article est d'établir le résultat suivant.

THÉORÈME 3.5. - On a

$$
\Delta\left(\omega^{W}, \omega^{V}, h^{\xi}\right)=0 \quad \text { dans } \quad P^{S} / P^{S, 0} .
$$

TOME $127-1999-\mathrm{N}^{\circ} 4$ 
Remarque 3.6. - D'après le théorème 3.3 , on sait que pour montrer le théorème 3.5 , il suffit de le montrer pour une forme $\omega^{W}$ donnée. En remplaçant $\omega^{W}$ par $\omega^{W}+\pi_{1}^{*} \omega^{V}$, on peut supposer que $\widetilde{\omega}^{W}$ est une $(1,1)$-forme réelle, fermée sur $W$, qui induit une métrique hermitienne sur $T Z$, et que de plus

$$
\omega^{W}=\widetilde{\omega}^{W}+\pi_{1}^{*} \omega^{V} .
$$

\section{c) Le cas où $\pi_{1}$ et $V$ sont projectives.}

Comme l'hypothèse (3.4) n'est pas satisfaite en général, on propose de montrer un résultat qui généralise le théorème 3.5. Dans le cas général, comme $E_{2}$ n'est pas isomorphe à $H\left(Z, \xi_{\mid Z}\right)$, le terme $\widetilde{\mathrm{ch}}\left(E_{2}, H\left(Z, \xi_{\mid Z}\right), h^{E_{2}}, h^{H\left(Z, \xi_{\mid Z}\right)}\right) \in P^{S} / P^{S, 0}$ n'est plus bien défini. Dans la suite, on donnera une façon naturelle de construire la classe de Bott-Chern $\widetilde{\operatorname{ch}}\left(E_{2}, H\left(Z, \xi_{\mid Z}\right), h^{E_{2}}, h^{H\left(Z, \xi_{\mid}\right)}\right)$quand $\pi_{1}$ et $V$ sont projectives.

Dans cette partie, on suppose que $\pi_{1}$ et $V$ sont projectives [BGS3, p. 337]. Alors $W$ est aussi projective.

THÉORÈme 3.8. - On peut définir la classe de Bott-Chern

$$
\widetilde{\operatorname{ch}}\left(E_{2}, H\left(Z, \xi_{\mid Z}\right), h^{E_{2}}, h^{H\left(Z, \xi_{\mid Z}\right)}\right) \in P^{S} / P^{S, 0}
$$

de manière «naturelle» de sorte que

$$
\begin{aligned}
\frac{\bar{\partial} \partial}{2 \pi i} \widetilde{c h}\left(E_{2}, H\left(Z, \xi_{\mid Z}\right), h^{E_{2}}, h^{H\left(Z, \xi_{\mid Z}\right)}\right) & \\
& =\operatorname{ch}\left(H\left(Z, \xi_{\mid Z}\right), h^{H\left(Z, \xi_{\mid Z}\right)}\right)-\operatorname{ch}\left(E_{2}, h^{E_{2}}\right) .
\end{aligned}
$$

THÉORÈME 3.9. - On suppose que $S$ est kählérienne. Soit $g^{T S}$ une métrique kählérienne sur TS. Soit $\sigma$ la section canonique de $\lambda^{-1}\left(E_{2}\right) \otimes \lambda\left(R^{\bullet} \pi_{3 *} \xi\right)$. Alors on $a$

$$
\begin{aligned}
\log \|\sigma\|_{\lambda^{-1}\left(E_{2}\right) \otimes \lambda\left(R^{\bullet} \pi_{3 *} \xi\right)}^{2} & \\
& =\int_{S} \operatorname{Td}\left(T S, g^{T S}\right) \widetilde{\operatorname{ch}}\left(E_{2}, H\left(Z, \xi_{\mid Z}\right), h^{E_{2}}, h^{H\left(Z, \xi_{\mid Z}\right)}\right) .
\end{aligned}
$$

Remarque 3.10. - Les preuves des théorèmes 3.8, 3.9 sont reportées au $\S 10$. On y expliquera précisément le mot «naturel».

Maintenant, on définit la forme $\Delta\left(\omega^{W}, \omega^{V}, h^{\xi}\right) \in P^{S} / P^{S, 0}$ comme en (3.6). Le but de cet article est d'établir aussi le résultat suivant.

THÉORÈme 3.11. - On a

$$
\Delta\left(\omega^{W}, \omega^{V}, h^{\xi}\right)=0 \quad \text { dans } \quad P^{S} / P^{S, 0} .
$$




\section{Preuve du théorème $\mathbf{3 . 5}$}

Dans ce paragraphe, on montre notre résultat essentiel, le théorème 3.5 , pour $\omega^{W}$ donnée par (3.11).

L'organisation de ce paragraphe est la même que dans [BerB, §4]. Comme dans [BerB, $\S 4]$, on donne des résultats intermédiaires dont les preuves sont données aux $\S \S 5-9$.

Ce paragraphe est organisé de la façon suivante. Dans a), on établit une identité $\sum_{k=1}^{4} I_{k}^{0}=\bar{\partial} \theta_{1}^{0}-\bar{\partial} \theta_{2}^{0}-\bar{\partial} \partial \theta_{3}^{0}$ de formes dans $P^{S}$. Le théorème 3.5 sera obtenu par passage à la limite à partir de cette identité. Dans b), on énonce des résultats intermédiaires qu'on va utiliser dans la preuve du théorème 3.5. Dans c), on calcule l'asymptotique des $I_{k}^{0}$. Dans d), on traite d'abord le terme à droite de l'équation ci-dessus. Comme un problème analogue a été résolu dans $[\mathrm{B} 4, \S 6]$, on omet les preuves. Le lecteur en trouvera le détail dans $[\mathrm{B} 4, \S 6]$. Enfin, on finit la preuve du théorème 3.5.

Dans ce paragraphe, on utilise les mêmes hypothèses et notations qu'au $\S \S 3 \mathrm{a}$ ) et $3 \mathrm{~b})$.

a) Une 1-forme fondamentale.

Soit $\omega_{T}^{W}(T>0)$ la $(1,1)$-forme réelle, fermée, $\mathcal{C}^{\infty}$ sur $W$ définie par

$$
\omega_{T}^{W}=\frac{1}{T^{2}} \widetilde{\omega}^{W}+\pi_{1}^{*} \omega^{V}
$$

Alors par (3.11), on a $\omega^{W}=\omega_{1}^{W}$.

On rappelle aussi que $g^{T Z}, g_{T}^{T Z}, g^{T X}, g^{T Y}$ sont les métriques induites par $\omega^{W}, \omega_{T}^{W}, \omega^{W}, \omega^{V}$ sur $T Z, T Z, T X, T Y$. Soit $*_{T}^{T Z}$ l'opérateur de Hodge agissant sur $\Lambda\left(T_{\mathbb{R}}^{*} Z\right)$ associé à la métrique $g_{T}^{T Z}$. On pose

$$
Q_{T}=-\left(*_{T}^{T Z}\right)^{-1} \frac{\partial}{\partial T}\left(*_{T}^{T Z}\right) .
$$

On rappelle que le fibré $\Lambda\left(T^{*(0,1)} Z\right) \otimes \xi$ est un $c\left(T_{\mathbb{R}} Z\right)$-module de Clifford. On note $c_{T}\left(\right.$. ) l'action de l'algèbre de Clifford de $\left(T_{\mathbb{R}} Z, g_{T}^{T_{\mathbb{R}}} Z\right)$ définie en (2.4).

Soit $\left\{e_{a}\right\}$ une base locale orthonormée de $T_{\mathbb{R}} Z$ pour la métrique $g_{T}^{T Z}$. Soit $\left\{g_{\alpha}\right\}$ une base de $T_{\mathbb{R}} S$, et soit $\left\{g^{\alpha}\right\}$ sa base duale de $T_{\mathbb{R}}^{*} S$.

DÉfinition 4.1. - Soit

$$
\begin{aligned}
M_{3, u, T}=-\frac{i}{\sqrt{2 u}}\left(\frac{\partial}{\partial T} \omega_{T}^{W}\right) & \left(g_{\alpha, 3, T}^{H}, e_{a}\right) g^{\alpha} c_{T}\left(e_{a}\right) \\
& -\frac{i}{2 u}\left(\frac{\partial}{\partial T} \omega_{T}^{W}\right)\left(g_{\alpha, 3, T}^{H}, g_{\beta, 3, T}^{H}\right) g^{\alpha} \wedge g^{\beta}-Q_{T} .
\end{aligned}
$$

Soit $\omega_{3, T}^{H \bar{H}}$ la forme correspondant à $\left(\pi_{3}, \omega_{T}^{W}\right)$ en (2.8). Soient $B_{3, u, T}, N_{3, u, T}$ les opérateurs définis en (2.7), (2.9) associés à $\omega_{T}^{W}, h^{\xi}$ et $\pi_{3}: W \rightarrow S$.

Tоме $127-1999-\mathrm{N}^{\circ} 4$ 
On fixe une racine carrée $\sqrt{2 \pi i}$ de $2 \pi i$. Soit $\varphi$ (resp. $\left.\varphi_{1}\right)$ l'homomorphisme de $\Lambda\left(T_{\mathbb{R}}^{*} S\right)$ dans $\Lambda\left(T_{\mathbb{R}}^{*} S\right)$ (resp. $\Lambda\left(T_{\mathbb{R}}^{*} V\right)$ dans $\left.\Lambda\left(T_{\mathbb{R}}^{*} V\right)\right)$ :

$$
\alpha \longmapsto(2 \pi i)^{-\frac{1}{2} \operatorname{deg} \alpha} \alpha
$$

DÉfinition 4.2. - Soit $\alpha_{u, T}$ la 1 -forme à valeurs dans $P^{S}$ sur $\mathbb{R}_{+}^{*} \times \mathbb{R}_{+}^{*}$

$$
\alpha_{u, T}=\mathrm{d} u \varphi \operatorname{Tr}_{s}\left[\frac{2}{u} N_{3, u^{2}, T} \exp \left(-B_{3, u^{2}, T}^{2}\right)\right]+\mathrm{d} T \varphi \operatorname{Tr}_{s}\left[M_{3, u^{2}, T} \exp \left(-B_{3, u^{2}, T}^{2}\right)\right]
$$

Par [BKö, th. 2.9], on a la proposition ci-dessous.

Proposition 4.3. - On a l'égalité suivante:

$$
\begin{aligned}
& \mathrm{d}_{u, T} \alpha_{u, T}=\frac{2}{u} \mathrm{~d} u \mathrm{~d} T \varphi \\
& \left\{\bar{\partial} \frac{\partial}{\partial b}\left\{\operatorname{Tr}_{s}\left[\left[B_{3, u^{2}, T}^{\prime}, N_{3, u^{2}, T}\right] \exp \left(-B_{3, u^{2}, T}^{2}-b M_{3, u^{2}, T}\right)\right]\right\}_{b=0}\right. \\
& \quad-\partial \frac{\partial}{\partial b}\left\{\operatorname{Tr}_{s}\left[\left[B_{3, u^{2}, T}^{\prime \prime}, N_{3, u^{2}, T}\right] \exp \left(-B_{3, u^{2}, T}^{2}-b M_{3, u^{2}, T}\right)\right]\right\}_{b=0} \\
& \left.\quad-\bar{\partial} \partial \frac{\partial}{\partial b}\left\{\operatorname{Tr}_{s}\left[N_{3, u^{2}, T} \exp \left(-B_{3, u^{2}, T}^{2}-b M_{3, u^{2}, T}\right)\right]\right\}_{b=0}\right\}
\end{aligned}
$$

Pour $0<\varepsilon<1,1 \leq A<+\infty, 1<T_{0}<+\infty$, soit $\Gamma=\Gamma_{\varepsilon, A, T_{0}}$ le contour orienté dans $\mathbb{R}_{+}^{*} \times \mathbb{R}_{+}^{*}$ :

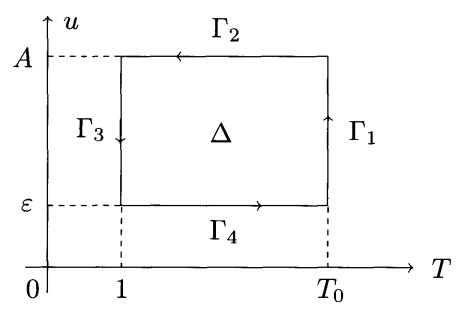

Dans la figure, $\Gamma$ est composé par les segments orientés $\Gamma_{1}, \ldots, \Gamma_{4}$. Alors le contour $\Gamma$ est le bord d'un domaine rectangulaire $\Delta$.

Pour $1 \leq k \leq 4$, on pose :

$$
I_{k}^{0}=\int_{\Gamma_{k}} \alpha_{u, T}
$$

BULLETIN DE LA SOCIÉTÉ MATHÉMATIQUE DE FRANCE 
Soient

$$
\left\{\begin{aligned}
\theta_{1}^{0}=(2 \pi i)^{-1 / 2} \int_{\Delta} & \frac{2}{u} \frac{\partial}{\partial b}\left\{\varphi \operatorname { T r } _ { s } \left[\left[B_{3, u^{2}, T}^{\prime}, N_{3, u^{2}, T}\right]\right.\right. \\
& \left.\left.\exp \left(-B_{3, u^{2}, T}^{2}-b M_{3, u^{2}, T}\right)\right]\right\}_{b=0} \mathrm{~d} u \mathrm{~d} T \\
\theta_{2}^{0}=(2 \pi i)^{-1 / 2} \int_{\Delta} \frac{2}{u} \frac{\partial}{\partial b}\left\{\varphi \operatorname { T r } _ { s } \left[\left[B_{3, u^{2}, T}^{\prime \prime}, N_{3, u^{2}, T}\right]\right.\right. & \\
& \left.\left.\exp \left(-B_{3, u^{2}, T}^{2}-b M_{3, u^{2}, T}\right)\right]\right\}_{b=0} \mathrm{~d} u \mathrm{~d} T \\
\theta_{3}^{0}=(2 \pi i)^{-1} \int_{\Delta} \frac{2}{u} \frac{\partial}{\partial b}\left\{\varphi \operatorname { T r } _ { s } \left[N_{3, u^{2}, T}\right.\right. & \left.\left.\exp \left(-B_{3, u^{2}, T}^{2}-b M_{3, u^{2}, T}\right)\right]\right\}_{b=0} \mathrm{~d} u \mathrm{~d} T .
\end{aligned}\right.
$$

THÉORÈME 4.4. - On a l'égalité :

$$
\sum_{k=1}^{4} I_{k}^{0}=\bar{\partial} \theta_{1}^{0}-\partial \theta_{2}^{0}-\bar{\partial} \partial \theta_{3}^{0} .
$$

Preuve. - C'est une conséquence immédiate de la proposition 4.3.

b) Des résultats intermédiaires.

On rappelle qu'on a les identifications de fibrés vectoriels $\mathcal{C}^{\infty}$ sur $W$,

$$
T Z \simeq T^{H} Z \oplus T X, \quad T^{H} Z \simeq \pi_{1}^{*} T Y .
$$

Ces identifications induisent une identification de fibrés $\mathcal{C}^{\infty} \mathbb{Z}$-gradués sur $W$

$$
\Lambda\left(T^{*(0,1)} Z\right) \simeq \pi_{1}^{*} \Lambda\left(T^{*(0,1)} Y\right) \widehat{\otimes} \Lambda\left(T^{*(0,1)} X\right) .
$$

Soient $N_{Z}, N_{X}, N_{Y}$ les opérateurs de nombre sur $\Lambda\left(T^{*(0,1)} Z\right), \Lambda\left(T^{*(0,1)} X\right)$, $\Lambda\left(T^{*(0,1)} Y\right)$. D'après $(4.9)$, les opérateurs $N_{X}, N_{Y}$ agissent sur $\Lambda\left(T^{*(0,1)} Z\right)$. Ils agissent aussi naturellement $\operatorname{sur} \Lambda\left(T^{*(0,1)} Z\right) \otimes \xi$ et sur $\Omega\left(Z, \xi_{\mid Z}\right)$. Naturellement,

$$
N_{Z}=N_{X}+N_{Y}
$$

Soient $B_{1, u}, B_{2, u}, B_{3, u}$ les superconnexions de Levi-Civita associées à $\left(\pi_{1}, \omega^{W}, h^{\xi}\right),\left(\pi_{2}, \omega^{V}, h^{R \pi_{1 *} \xi}\right),\left(\pi_{3}, \omega^{W}, h^{\xi}\right)$ définies à la définition 2.3. Soit $\omega_{1}^{H \bar{H}}$, $\widetilde{\omega}_{1}^{H \bar{H}}, \omega_{2}^{H \bar{H}}, \omega_{3}^{H \bar{H}}$ les formes associées à $\left(\pi_{1}, \omega^{W}\right),\left(\pi_{1}, \widetilde{\omega}^{W}\right),\left(\pi_{2}, \omega^{V}\right),\left(\pi_{3}, \omega^{W}\right)$ définies en (2.8). Soient $N_{1, u}, \widetilde{N}_{1, u}, N_{2, u}, N_{3, u}$ les opérateurs associés à $\left(\pi_{1}, \omega^{W}\right)$, $\left(\pi_{1}, \widetilde{\omega}^{W}\right),\left(\pi_{2}, \omega^{V}\right),\left(\pi_{3}, \omega^{W}\right)$ définis à la définition 2.4 .

Maintenant, on expose des résultats qui vont être utilisés dans la preuve du théorème 3.5. Les preuves des résultats seront données aux $\S \S 5-9$.

TOME $127-1999-\mathrm{N}^{\circ} 4$ 
Dans la suite, on met sur les formes $\mathcal{C}^{\infty}$ sur $S$ la topologie de la convergence uniforme des formes et de leurs dérivées sur les parties compactes de $S$. Pour éviter de noter explicitement des constantes qui dépendent du compact considéré, on suppose, pour simplifier, que $S$ est compacte.

THÉORÈME 4.5.

(i) Pour tout $u>0$,

$$
\lim _{T \rightarrow+\infty} \varphi \operatorname{Tr}_{s}\left[N_{3, u, T} \exp \left(-B_{3, u, T}^{2}\right)\right]=\varphi \operatorname{Tr}_{s}\left[N_{2, u} \exp \left(-B_{2, u}^{2}\right)\right]
$$

(ii) Pour tout $u>0$, il existe $C>0, \delta>0$ tels que pour $T \geq 1$,

$$
\begin{aligned}
& \mid \varphi \operatorname{Tr}_{s}\left[M_{3, u, T} \exp \left(-B_{3, u, T}^{2}\right)\right] \\
& \quad-\frac{2}{T} \varphi \operatorname{Tr}_{s}\left[\left(N_{X}-\operatorname{dim} X\right) \exp \left(-B_{2, u}^{2}\right)\right] \mid \leq \frac{C}{T^{\delta+1}} .
\end{aligned}
$$

ThÉORÈme 4.6. - Il existe $C>0, T_{0}>1$ tels que, pour tout $T \geq T_{0}, u \geq 1$,

$$
\left|\varphi \operatorname{Tr}_{s}\left[N_{3, u, T} \exp \left(-B_{3, u, T}^{2}\right)\right]\right| \leq \frac{C}{u} .
$$

Pour $s \in S$, soit $\langle,\rangle_{T, s}$ le produit hermitien (2.2) sur $\Omega\left(Z_{s}, \xi_{\mid Z_{s}}\right)$ associé à $g_{T}^{T Z}, h^{\xi}$. Soit $\bar{\partial}_{T}^{Z_{s}, *}$ l'adjoint formel de $\bar{\partial}^{Z_{s}}$ pour le produit hermitien $\langle,\rangle_{T, s}$ $\operatorname{sur} \Omega\left(Z_{s}, \xi_{\mid Z_{s}}\right)$. Soient

$$
D_{T}^{Z}=\bar{\partial}^{Z}+\bar{\partial}_{T}^{Z, *}, \quad D^{Y}=\bar{\partial}^{Y}+\bar{\partial}^{Y, *}, \quad D^{X}=\bar{\partial}^{X}+\bar{\partial}^{X, *} .
$$

Soit $P^{\operatorname{ker} D_{T}^{Z}}$ la projection orthogonale de $\Omega\left(Z, \xi_{\mid Z}\right)$ sur ker $D_{T}^{Z}$ pour le produit hermitien $\langle,\rangle_{T}$. Dans la suite, on note $P^{\operatorname{ker} D_{T}^{Z}}$ par $P_{T}^{H\left(Z, \xi_{\mid Z}\right)}$. Alors, par la théorie de Hodge, l'application

$$
s \in \operatorname{ker} D^{Z} \longmapsto P_{T}^{H\left(Z, \xi_{\mid Z}\right)} s \in \operatorname{ker} D_{T}^{Z}
$$

est l'identification canonique de $\operatorname{ker} D^{Z}$ à $\operatorname{ker} D_{T}^{Z}$.

Soit $h_{T}^{H\left(Z, \xi_{\mid Z}\right)}$ la métrique sur $H\left(Z, \xi_{\mid Z}\right)$ associée à $g_{T}^{T Z}, h^{\xi}$ définie au $\S 2 \mathrm{~b})$. Soient $\nabla_{T}^{H\left(Z, \xi_{\mid Z}\right)}, \nabla^{E_{2}}$ les connexions holomorphes hermitiennes sur $\left(H\left(Z, \xi_{\mid Z}\right), h_{T}^{H\left(Z, \xi_{\mid Z}\right)}\right),\left(E_{2}, h^{E_{2}}\right)$. On pose

$$
Q_{T}^{H\left(Z, \xi_{\mid Z}\right)}=P_{T}^{H\left(Z, \xi_{\mid Z}\right)} Q_{T} P_{T}^{H\left(Z, \xi_{\mid Z}\right)} .
$$

Proposition 4.7. - Quand $T \rightarrow+\infty$, on a

$$
\nabla_{T}^{H\left(Z, \xi_{\mid Z}\right), 2}=\nabla^{E_{2}, 2}+O\left(\frac{1}{T}\right)
$$


De plus, on a dans $P^{S} / P^{S, 0}$

$$
\begin{aligned}
\int_{1}^{+\infty}\left\{\varphi \operatorname{Tr}_{s}\left[Q_{T}^{H\left(Z, \xi_{\mid Z}\right)} \exp \left(-\nabla_{T}^{H\left(Z, \xi_{\mid Z}\right), 2}\right)\right]\right. \\
\left.+\varphi \operatorname{Tr}_{s}\left[\frac{2\left(N_{X}-\operatorname{dim} X\right)}{T} \exp \left(-\nabla^{E_{2}, 2}\right)\right]\right\} \mathrm{d} T \\
=-\widetilde{\operatorname{ch}}\left(E_{2}, H\left(Z, \xi_{\mid Z}\right), h^{E_{2}}, h^{H\left(Z, \xi_{\mid Z}\right)}\right) .
\end{aligned}
$$

ThÉorème 4.8. - Pour tout $T>0$, on a

$$
\begin{aligned}
\lim _{\varepsilon \rightarrow 0} \varphi \operatorname{Tr}_{s} & {\left[\frac{1}{\varepsilon} M_{3, \varepsilon^{2}, T / \varepsilon} \exp \left(-B_{3, \varepsilon^{2}, T / \varepsilon}^{2}\right)\right] } \\
& =\frac{2}{T} \int_{Y} \operatorname{Td}\left(T Y, g^{T Y}\right) \varphi_{1} \operatorname{Tr}_{s}\left[\left(\widetilde{N}_{1, T^{2}}-\operatorname{dim} X\right) \exp \left(-B_{1, T^{2}}^{2}\right)\right] .
\end{aligned}
$$

Si $J^{\prime} \in \Lambda\left(T_{\mathbb{C}}^{*} V\right) \widehat{\otimes} \mathbb{C}(\mathrm{d} u), J^{\prime}$ peut s'écrire

$$
J^{\prime}=J_{0}^{\prime}+\mathrm{d} u J_{1}^{\prime} \quad \text { avec } \quad J_{0}^{\prime}, J_{1}^{\prime} \in \Lambda\left(T_{\mathbb{C}}^{*} V\right) .
$$

On pose

$$
\left[J^{\prime}\right]^{\mathrm{d} u}=J_{1}^{\prime} .
$$

D'après [BGS2, th. 2.16], on peut poser :

$$
E_{1,0}=\lim _{u \rightarrow 0} \operatorname{Tr}_{s}\left[\tilde{N}_{1, u} \exp \left(-B_{1, u}^{2}+\mathrm{d} u\left(2 u \frac{\partial B_{1, u}}{\partial u}\right)\right)\right]^{\mathrm{d} u} .
$$

THÉORÈME 4.9.

(i) Il existe une forme $\mu_{0}(T), \mathcal{C}^{\infty}$ sur $S$ telle que pour $T$ fixé, quand $u \rightarrow 0$,

$$
\operatorname{Tr}_{s}\left[u M_{3, u, T} \exp \left(-B_{3, u, T}^{2}+\mathrm{d} u \frac{\partial}{\partial u} B_{3, u, T}\right)\right]^{\mathrm{d} u}=\mu_{0}(T)+O(u) .
$$

(ii) Il existe une forme $E, \mathcal{C}^{\infty}$ sur $S$ telle que, quand $T \rightarrow+\infty$

$$
\mu_{0}(T)=\frac{1}{T} E+O\left(\frac{1}{T^{2}}\right)
$$

avec

$$
\varphi \mathrm{d}^{S} E=(2 \pi i)^{-\frac{1}{2}} \mathrm{~d}^{S} \int_{Y} \mathrm{Td}\left(T Y, g^{T Y}\right) \varphi_{1} E_{1,0}
$$

On rappelle aussi que $\nabla_{T}^{T Z}$ est la connexion holomorphe hermitienne sur $\left(T Z, g_{T}^{T Z}\right)$. Soit $R_{T}^{T Z}$ sa courbure.

TOME $127-1999-\mathrm{N}^{\circ} 4$ 
ThÉORÈme 4.10. - Il existe $C>0$ tel que, pour tout $\varepsilon \in] 0,1], \varepsilon \leq T \leq 1$,

$$
\begin{aligned}
\mid \varphi \operatorname{Tr}_{s}[ & \left.\frac{1}{\varepsilon} M_{3, \varepsilon^{2}, T / \varepsilon} \exp \left(-B_{3, \varepsilon^{2}, T / \varepsilon}^{2}\right)\right] \\
& \quad-\frac{2}{T^{3}} \int_{Z} \frac{\widetilde{\omega}^{W}}{2 \pi} \operatorname{Td}\left(T Z, g_{T / \varepsilon}^{T Z}\right) \operatorname{ch}\left(\xi, h^{\xi}\right) \\
& +\int_{Z} \frac{\partial}{\partial b} \operatorname{Td}\left(\frac{-R_{T / \varepsilon}^{T Z}}{2 \pi i}-b\left(g_{T / \varepsilon}^{T Z}\right)^{-1} \frac{\partial}{\partial T}\left(g_{T / \varepsilon}^{T Z}\right)\right)_{b=0} \operatorname{ch}\left(\xi, h^{\xi}\right) \\
& \quad+\frac{1}{\varepsilon} \varphi \mathrm{d}^{S} \mu_{0}\left(\frac{T}{\varepsilon}\right) \mid \leq C .
\end{aligned}
$$

ThÉORÈme 4.11. - Il existe $\delta \in] 0,1], C>0$ tels que, pour tout $\varepsilon \in] 0,1]$, $T \geq 1$,

$$
\begin{aligned}
\mid \varphi \operatorname{Tr}_{s}\left[\frac{1}{\varepsilon} M_{3, \varepsilon^{2}, T / \varepsilon} \exp (\right. & \left.\left.-B_{3, \varepsilon^{2}, T / \varepsilon}^{2}\right)\right] \\
& -\frac{2}{T} \varphi \operatorname{Tr}_{s}\left[\left(N_{X}-\operatorname{dim} X\right) \exp \left(-B_{2, \varepsilon^{2}}^{2}\right)\right] \mid \leq \frac{C}{T^{1+\delta}}
\end{aligned}
$$

Remarque 4.12. - Les théorèmes 4.5, 4.6 seront démontrés au $\S 5$, la proposition 4.7 au $\S 6$, le théorème 4.8 au $\S 7$, les théorèmes 4.9 et 4.10 au $\S 8$, le théorème 4.11 au $\S 9$. L'hypothèse (3.4) ne sert qu'aux preuves des théorèmes $4.5-4.7$.

\section{c) L'asymptotique des $\boldsymbol{I}_{\boldsymbol{k}}^{\mathbf{0}}$.}

Dans cette partie, on va procéder comme en $[\mathrm{BerB}, \S 4(\mathrm{c})]$, i.e. on calcule l'asymptotique de $I_{k}^{0}$ quand $A \rightarrow+\infty, T \rightarrow+\infty$ et $\varepsilon \rightarrow 0$. On n'indiquera que la différence avec $[\operatorname{BerB}, \S 4(\mathrm{c})]$, et on omet les détails des calculs

1) Le terme $I_{1}^{0}$. - Soient

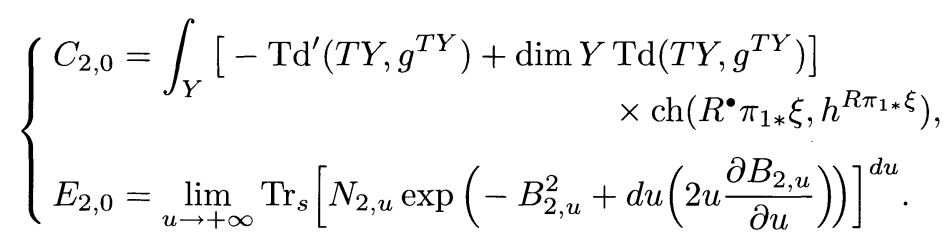

En utilisant [BGS2, th. 2.16], [B4, (2.50)], les théorèmes 4.5 et 4.6, après avoir passé à la limite $A \rightarrow+\infty, T_{0} \rightarrow+\infty, \varepsilon \rightarrow 0$, on obtient

$$
I_{1}^{3}=-T_{2}\left(\omega^{V}, h^{R \pi_{1 *} \xi}\right)+\Gamma^{\prime}(1)\left(C_{2,0}-\frac{1}{2} \varphi d^{S} E_{2,0}\right) .
$$


2) Le terme $I_{2}^{0}$. - En utilisant la proposition 4.7 , on a :

$$
I_{2}^{3}=-\widetilde{\operatorname{ch}}\left(E_{2}, H\left(Z, \xi_{\mid Z}\right), h^{E_{2}}, h^{H\left(Z, \xi_{\mid Z}\right)}\right) \quad \text { dans } \quad P^{S} / P^{S, 0} .
$$

3) Le terme $I_{3}^{0}$. - Ce terme a déjà été traité en $[\mathrm{B} 4,(6.54)-(6.56)]$. Soient

$$
\left\{\begin{array}{l}
C_{3,0}=\int_{Z}\left[-\operatorname{Td}^{\prime}\left(T Z, g^{T Z}\right)+\operatorname{dim} Z \operatorname{Td}\left(T Z, g^{T Z}\right)\right] \operatorname{ch}\left(\xi, h^{\xi}\right), \\
E_{3,0}=\lim _{u \rightarrow 0} \operatorname{Tr}_{s}\left[N_{3, u} \exp \left(-B_{3, u}^{2}+d u\left(2 u \frac{\partial B_{3, u}}{\partial u}\right)\right)\right]^{d u} .
\end{array}\right.
$$

Après avoir passé à la limite $A \rightarrow+\infty, T_{0} \rightarrow+\infty, \varepsilon \rightarrow 0$, on obtient

(4.29) $\quad I_{3}^{3}=T_{3}\left(\omega^{W}, h^{\xi}\right)$

$$
+\Gamma^{\prime}(1)\left\{\frac{1}{2} \varphi d^{S} E_{3,0}-C_{3,0}+\varphi \operatorname{Tr}_{s}\left[N_{Z} \exp \left(-\nabla^{H\left(Z, \xi_{\mid Z}\right), 2}\right)\right]\right\} .
$$

4) Le terme $I_{4}^{0}$. - La différence principale avec [BerB, p. 115-121] est qu'on doit contrôler le terme $\varphi d^{S} \mu_{0}(T)$ dans le théorème 4.10 , mais ce terme est déjà traité au théorème 4.9 .

Évidemment, quand $T \rightarrow+\infty$

$$
\left(g_{T}^{T Z}\right)^{-1} \frac{\partial}{\partial T}\left(g_{T}^{T Z}\right)=-\frac{2 P^{T X}}{T}+O\left(\frac{1}{T^{3}}\right) .
$$

Par le théorème 1.4, on déduit facilement que quand $T \rightarrow+\infty$,

$$
\left\{\begin{aligned}
\frac{\partial}{\partial b} \operatorname{Td}\left(\frac{-R_{T}^{T Z}}{2 \pi i}\right. & \left.-b\left(g_{T}^{T Z}\right)^{-1} \frac{\partial}{\partial T}\left(g_{T}^{T Z}\right)\right)_{b=0} \\
& =\frac{2}{T} \pi_{1}^{*}\left(\operatorname{Td}\left(T Y, g^{T Y}\right)\right) \operatorname{Td}^{\prime}\left(T X, g^{T X}\right)+O\left(\frac{1}{T^{3}}\right) \\
\operatorname{Td}\left(\frac{-R_{T}^{T Z}}{2 \pi i}\right) & =\pi_{1}^{*}\left(\operatorname{Td}\left(T Y, g^{T Y}\right)\right) \operatorname{Td}\left(T X, g^{T X}\right)+O\left(\frac{1}{T^{2}}\right) .
\end{aligned}\right.
$$

En utilisant [B4, th. 2.17, 2.20, 2.24], les théorèmes 4.8, 4.9, 4.10 et (4.32), après être passé à la limite $A \rightarrow+\infty, T_{0} \rightarrow+\infty, \varepsilon \rightarrow 0$, on obtient dans $P^{S} / P^{S, 0}$

$$
\begin{array}{r}
I_{4}^{3}=\int_{Z} \widetilde{\operatorname{Td}}\left(T Z, T Y, g^{T Z}, g^{T Y}\right) \operatorname{ch}\left(\xi, h^{\xi}\right)-\int_{Y} \operatorname{Td}\left(T Y, g^{T Y}\right) T_{1}\left(\omega^{W}, h^{\xi}\right) \\
-\Gamma^{\prime}(1) \int_{Y} \operatorname{Td}\left(T Y, g^{T Y}\right)\left\{\pi _ { 1 * } \left[\left(\operatorname{Td}^{\prime}\left(T X, g^{T X}\right)-\operatorname{dim} X \operatorname{Td}\left(T X, g^{T X}\right)\right)\right.\right. \\
\left.\left.\operatorname{ch}\left(\xi, h^{\xi}\right)\right]+\operatorname{ch}^{\prime}\left(R^{\bullet} \pi_{1 *} \xi, h^{R \pi_{1 *}}\right)\right\} .
\end{array}
$$

TOME $127-1999-\mathrm{N}^{\circ} 4$ 


\section{d) Preuve du théorème 3.5.}

Comme dans [B4, $\S \S 6.6-6.8]$, on établit l'équation

$$
\sum_{k=1}^{4} I_{k}^{3}=\bar{\partial} \theta_{1}^{3}-\partial \theta_{2}^{3}-\bar{\partial} \partial \theta_{3}^{3} .
$$

Bien sûr, on doit étudier en détail le terme à droite de (4.8). Comme la situation est plus simple que dans $[\mathrm{B} 4, \S 6]$, on ne le fait pas; le lecteur peut trouver les détails dans $[\mathrm{B} 4, \S 6]$.

On remarque aussi que, si $S$ est compacte et kählérienne, l'espace $P^{S, 0}$ est fermé dans $P^{S}$ pour la topologie de convergence uniforme. Dans ce cas, on voit facilement que, comme $\sum_{k=1}^{4} I_{k}^{0} \in P^{S, 0}$, on a $\sum_{k=1}^{4} I_{k}^{3} \in P^{S, 0}$.

Par (4.27), (4.28), (4.30), (4.33), le coefficient de $\Gamma^{\prime}(1)$ du terme à gauche de (4.34) est nul dans $P^{S} / P^{S, 0}$.

Par (4.27), (4.28), (4.30), (4.33),(4.34), on en déduit le théorème 3.5 .

\section{L'asymptotique des supertraces contenant l'opérateur $\exp \left(-B_{3, u, T}^{2}\right)$ pour $u$ ou $T$ tendant vers l'infini}

Le but de ce paragraphe est de montrer les théorèmes 4.5 et 4.6. C'est une extension de [BerB, $\S 5]$, qui traite le cas où $S$ est un point.

Dans [BerB, $\S 5], T D_{T}^{X}+D_{T}^{H}$ est un opérateur différentiel elliptique. La nouvelle difficulté est que $B_{3, u, T}$ est une superconnexion, et que seul le carré $B_{3, u, T}^{2}$ est un opérateur elliptique standard agissant sur chaque fibre. Un problème similaire a déjà été considéré dans $[\mathrm{B} 4, \S 9]$. C'est pourquoi on va toujours se ramener à la situation traitée dans $[\mathrm{B} 4, \S 9]$.

Ce paragraphe est organisé de la façon suivante. Dans a), on rappelle des notations de [BerB, $\S 5]$. Dans b), on remplace la superconnexion $B_{3, u, T} \operatorname{par} A_{u, T}$. Soit $A_{T}=A_{1, T}$; on obtient alors l'asymptotique de $A_{T}$ quand $T \rightarrow+\infty$. Dans c), on obtient l'asymptotique de $A_{T}^{2}$ écrite sous forme matricielle relativement à un scindement de $E_{0}=\Omega\left(Z, \xi_{\mid Z}\right)$. Dans d), on décrit le spectre de $A_{u, T}^{2}$. Dans e), on énonce deux résultats intermédiaires, d'où les théorèmes 4.5 et 4.6 peuvent être facilement déduits. Le reste du paragraphe est consacré à la preuve de ces deux résultats intermédiaires.

Dans f), on donne des estimations pour la résolvante de $A_{T}^{2}$. Dans g), on obtient une estimation uniforme pour le noyau de $F_{u}\left(A_{T}^{2}\right)$. Dans h), on calcule l'asymptotique de l'opérateur $F_{u}\left(A_{T}^{2}\right)$ quand $T \rightarrow+\infty$. Dans i), on montre le premier résultat intermédiaire et (4.12). Dans $\mathrm{j}$ ), on montre le deuxième résultat intermédiaire. 
Dans ce paragraphe, on suppose que la forme $\omega^{W}$ sur $W$ est donné par (3.11), et que le fibré holomorphe $\xi$ vérifie l'hypothèse d'acyclicité (3.4). On utilise les mêmes notations qu'aux $\S \S 1 \mathrm{~b}), 3$ et 4 .

\section{a) Le cas où $S$ est un point.}

Dans la suite, on rappelle des notations de [BerB, §5] en fixant un point $s \in S$. Alors $\pi_{1}: Z \rightarrow Y$ est une submersion holomorphe de fibre compacte $X$.

On rappelle que $h \in \operatorname{End}\left(\pi_{1}^{*} T Y\right)$ est défini en (1.32).

Définition 5.1. - Pour $T \geq 1$ et $\alpha \in \pi_{1}^{*}\left(\Lambda\left(T^{*(0,1)} Y\right)\right)$, on définit $a_{T} \alpha \in$ $\pi_{1}^{*}\left(\Lambda\left(T^{*(0,1)} Y\right)\right)$ de la manière suivante :

$$
\begin{aligned}
& v_{1}, \cdots, v_{p} \in \overline{T Y} \longmapsto \\
& \quad a_{T} \alpha\left(v_{1}, \cdots, v_{p}\right)=\alpha\left(\left(1+\frac{\bar{h}}{T^{2}}\right)^{-1 / 2} v_{1}, \ldots,\left(1+\frac{\bar{h}}{T^{2}}\right)^{-1 / 2} v_{p}\right) .
\end{aligned}
$$

Alors $a_{T}$ agit sur

$$
\Lambda\left(T^{*(0,1)} Z\right) \simeq \pi_{1}^{*}\left(\Lambda\left(T^{*(0,1)} Y\right)\right) \widehat{\otimes} \Lambda\left(T^{*(0,1)} X\right)
$$

(voir (4.9)) comme $a_{T} \widehat{\otimes} 1$.

Définition 5.2. - Pour $T \geq 1$, soient

$$
B_{T}=\operatorname{det}^{1 / 2}\left(1+\frac{h}{T^{2}}\right) a_{T}, \quad C_{T}=T^{N_{X}-\operatorname{dim} X} B_{T}
$$

Définition 5.3. - Pour a $\in Y$, soient $E_{a}, E_{0}$ les espaces vectoriels des sections $\mathcal{C}^{\infty}$ de $\Lambda\left(T^{*(0,1)} Z\right) \otimes \xi$ sur $X_{a}, Z$. On a donc

$$
E_{a}=\Lambda\left(T^{*(0,1)} Y\right)_{a} \widehat{\otimes} \Omega\left(X_{a}, \xi_{\mid X_{a}}\right) .
$$

Soit $\mathrm{d} v_{Z}$ (resp. $\mathrm{d} v_{X}, \mathrm{~d} v_{Y}$ ) la forme de volume sur $Z$ (resp. $X, Y$ ) associée à la métrique $\pi_{1}^{*} g^{T Y} \oplus g^{T X}$ sur $T Z=\pi_{1}^{*} T Y \oplus T X$ (resp. $g^{T X}$ sur $T X, g^{T Y}$ sur $T Y$ ). Soit \langle\rangle$_{\Lambda\left(T^{*(0,1)} Z\right) \otimes \xi, \infty}$ le produit hermitien sur $\Lambda\left(T^{*(0,1)} Z\right) \otimes \xi$ associé aux métriques $\pi_{1}^{*} g^{T Y} \oplus g^{T X}, h^{\xi}$ sur $T Z=\pi_{1}^{*} T Y \oplus T X, \xi$. Pour $a \in Y$ et $s, s^{\prime} \in E_{a}$, on pose

$$
\left\langle s, s^{\prime}\right\rangle_{\mid E_{a}}=(2 \pi)^{-\operatorname{dim} X} \int_{X_{a}}\left\langle s, s^{\prime}\right\rangle_{\Lambda\left(T^{*}(0,1) Z\right) \otimes \xi, \infty} \mathrm{d} v_{X}
$$

Définition 5.4. - Pour $\mu \in \mathbb{R}$, soient $E_{0}^{\mu}, E_{1}^{\mu}$ les espaces de Sobolev d'ordre $\mu$ des sections de $\Lambda\left(T^{*(0,1)} Z\right) \otimes \xi$, Ker $D^{X}$ sur $Z, Y$.

Soit \langle\rangle$_{E_{0}^{0}}=\langle\rangle_{\infty}$ le produit hermitien sur $E_{0}^{0}$ associé aux métriques $\pi_{1}^{*} g^{T Y} \oplus g^{T X}, h^{\xi}$ sur $T Z=\pi_{1}^{*} T Y \oplus T X, \xi$ défini en (2.2). Soit \|\|$_{E_{0}^{0}}$ la norme correspondante sur $E_{0}^{0}$. Soit $E_{1}^{0, \perp}$ l'espace orthogonal à $E_{1}^{0}$ dans $E_{0}^{0}$.

TOME $127-1999-\mathrm{N}^{\circ} 4$ 
Soit $\Omega\left(Y, \Omega\left(X, \xi_{\mid X}\right)_{\mid Y}\right)$ l'espace vectoriel des sections $\mathcal{C}^{\infty}$ de $\Lambda\left(T^{*(0,1)} Y\right)$ $\widehat{\otimes} \Omega\left(X, \xi_{\mid X}\right)$ sur $Y$. Donc $\Omega\left(Y, \Omega\left(X, \xi_{\mid X}\right)_{\mid Y}\right)$ est l'espace vectoriel des sections $\mathcal{C}^{\infty}$ de $\pi_{1}^{*}\left(\Lambda\left(T^{*(0,1)} Y\right)\right) \widehat{\otimes} \Lambda\left(T^{*(0,1)} X\right) \otimes \xi$ sur $Z$. Par l'identification (4.9), on a une identification d'espaces vectoriels

$$
\Omega\left(Z, \xi_{\mid Z}\right) \simeq \Omega\left(Y, \Omega\left(X, \xi_{\mid X}\right)_{\mid Y}\right)
$$

Soit $\nabla_{\mid Y}{ }^{\Omega(X, \xi \mid X)}$ la restriction de la connexion $\nabla^{\Omega(X, \xi \mid X)}$ sur $\Omega\left(X, \xi_{\mid X}\right)$ à $Y$. Sous l'identification (5.5), $\nabla_{\mid Y} \Omega(X, \xi \mid X)^{\prime \prime}$ agit naturellement sur $\Omega\left(Z, \xi_{\mid Z}\right)$. De même $\bar{\partial}^{X}$ et $D^{X}$ agissent sur $\Omega\left(Z, \xi_{\mid Z}\right)$. Désormais, on pose

$$
\bar{\partial}^{H}=\nabla_{\mid Y} \Omega(X, \xi \mid X)^{\prime \prime} .
$$

Soit $\bar{\partial}_{\infty}^{H *}$ l'adjoint formel de $\bar{\partial}^{H}$ relativement à \langle\rangle$_{\infty}$.

DÉfinition 5.5. - Pour tout $T \in[1,+\infty]$, on pose

$$
\begin{cases}\bar{\partial}_{T}^{X}=B_{T} \bar{\partial}^{X} B_{T}^{-1}, & \bar{\partial}_{T}^{X *}=B_{T}^{-1} \bar{\partial}^{X *} B_{T} \\ \bar{\partial}_{T}^{H}=B_{T} \bar{\partial}^{H} B_{T}^{-1}, & \bar{\partial}_{T}^{H *}=B_{T}^{-1} \bar{\partial}_{\infty}^{H *} B_{T} \\ D_{T}^{X}=\bar{\partial}_{T}^{X}+\bar{\partial}_{T}^{X *}, & D_{T}^{H}=\bar{\partial}_{T}^{H}+\bar{\partial}_{T}^{H *}\end{cases}
$$

DÉfinition 5.6. - Pour $a \in Y, T \in[1,+\infty]$, soit $p_{T, a}$ la projection orthogonale de $E_{a}$ sur $\operatorname{ker} D_{T, a}^{X}$ associée au produit hermitien \langle\rangle$_{E_{a}}$. On pose $p_{T, a}^{\perp}=1-p_{T, a}$.

Soit $E_{1}$ l'espace vectoriel des sections $\mathcal{C}^{\infty}$ de $\operatorname{ker} D^{X}$ sur $Y$. Pour $T \in[1,+\infty]$, soit $E_{1, T}$ l'espace vectoriel des sections $\mathcal{C}^{\infty}$ de $\operatorname{ker} D_{T}^{X}$ sur $Y$, et soit $q_{T}$ : $E_{1, \infty} \rightarrow E_{1, T}$ l'application linéaire

$$
s \in E_{1, \infty} \longmapsto q_{T} s=p_{T} B_{T} s \in E_{1, T} .
$$

Soit $q_{T}^{*}$ l'adjoint de $q_{T}$. On pose

$$
r_{T}=\left(q_{T}^{*} q_{T}\right)^{1 / 2}, \quad J_{T}=q_{T} r_{T}^{-1}
$$

Alors $J_{T}: E_{1, \infty} \rightarrow E_{1, T}$ est un isométrie linéaire.

Dans la suite, si $S_{T}$ (avec $T \in[1,+\infty]$ ) est une famille d'opérateurs différentiels, on écrit que, quand $T \rightarrow+\infty$,

$$
S_{T}=S_{\infty}+O\left(\frac{1}{T^{\ell}}\right) \quad \text { avec } \quad \ell \in \mathbb{Z}
$$

si pour tout entier $k \in \mathbb{N}$, il existe $C>0$ tel que pour $T \geq 1$, les normes des coefficients de $S_{T}-S_{\infty}$ et de leurs dérivées d'ordre $\leq k$ sont dominées par $C T^{-\ell}$. 


\section{b) L'asymptotique de la superconnexion $A_{T}$ quand $T$ tend vers l'infini.}

Pour $U \in T_{\mathbb{R}} Z$, on note $c(U)$ l'action de l'algèbre de Clifford sur $\Lambda\left(T^{*(0,1)} Z\right)$ associée à $\left(T Z, \pi_{1}^{*} g^{T Y} \oplus g^{T X}\right)$. Alors pour

$$
U=U^{T^{H} Z}+U^{T X} \in T_{\mathbb{R}} Z
$$

avec $U^{T^{H} Z} \in T_{\mathbb{R}}^{H} Z$ et $U^{T X} \in T_{\mathbb{R}} X$, on a

$$
C_{T} c_{T}\left(\left(1+\frac{h}{T^{2}}\right)^{-1 / 2} U^{T^{H} Z}+T U^{T X}\right) C_{T}^{-1}=c\left(U^{T^{H}} Z\right)+c\left(U^{T X}\right) .
$$

Soit $g_{\alpha}$ une base de $T_{\mathbb{R}} S$; soient $e_{i}, w_{i}$ des bases orthonormées de $\left(T_{\mathbb{R}} X, g^{T_{\mathbb{R}}} X\right)$ et $\left(T X, g^{T X}\right)$; soient $f_{\ell}, \theta_{\ell}$ des bases orthonormées de $\left(T_{\mathbb{R}} Y, g^{T_{\mathbb{R}} Y}\right)$ et $\left(T Y, g^{T Y}\right)$; soient $g^{\alpha}, e^{i}, w^{i}, f^{\ell}, \theta^{\ell}$ les bases duales associées. Soient enfin

$$
f_{\ell, T}=\left(1+\frac{h}{T^{2}}\right)^{-1 / 2} f_{\ell, 1}^{H}, \quad \theta_{\ell, T}=\left(1+\frac{h}{T^{2}}\right)^{-1 / 2} \theta_{\ell, 1}^{H} .
$$

Alors $T e_{i}, f_{\ell, T}$ (resp. $\left.T w_{i}, \theta_{\ell, T}\right)$ est une base orthonormée de $\left(T_{\mathbb{R}} Z, g_{T}^{T_{\mathbb{R}}} Z\right.$ ) (resp. $\left.\left(T Z, g_{T}^{T Z}\right)\right)$.

On rappelle que $\nabla_{T}^{T Z}, \nabla^{T X}, \nabla^{T Y}, \nabla^{\xi}$ sont les connexions holomorphes hermitiennes sur

$$
\left(T Z, g_{T}^{T Z}\right), \quad\left(T X, g^{T X}\right), \quad\left(T Y, g^{T Y}\right), \quad\left(\xi, h^{\xi}\right) .
$$

Soit $\nabla^{\Lambda\left(T^{*(0,1)} X\right)}, \nabla^{\Lambda\left(T^{*(0,1)} Y\right)}$ les connexions sur $\Lambda\left(T^{*(0,1)} X\right), \Lambda\left(T^{*(0,1)} Y\right)$ induites par $\nabla^{T X}, \nabla^{T Y}$. Soient $R_{T}^{T Z}, R^{T X}, R^{T Y}, R^{\Lambda\left(T^{*(0,1)} X\right)}, L^{\xi}$ les courbures associées à $\nabla_{T}^{T Z}, \nabla^{T X}, \nabla^{T Y}, \nabla^{\Lambda\left(T^{*(0,1)} X\right)}, \nabla^{\xi}$.

Soit $\nabla^{\pi_{1}^{*} T Y}=\pi_{1}^{*} \nabla^{T Y}$; alors ${ }^{0} \nabla^{T Z}=\nabla^{\pi_{1}^{*} T Y} \oplus \nabla^{T X}$ est une connexion sur

$$
T Z \simeq \pi_{1}^{*} T Y \oplus T X
$$

Soient $\nabla_{T}^{\Lambda\left(T^{*(0,1)} Z\right)},{ }^{0} \nabla^{\Lambda\left(T^{*(0,1)} Z\right)}$ les connexions induites par $\nabla_{T}^{T Z},{ }^{0} \nabla^{T Z}$ sur $\Lambda\left(T^{*(0,1)} Z\right)$. Soient $\nabla_{T}^{\Lambda\left(T^{*(0,1)} Z\right) \otimes \xi},{ }^{0} \nabla^{\Lambda\left(T^{*(0,1)} Z\right) \otimes \xi}$ les connexions sur $\Lambda\left(T^{*(0,1)} Z\right)$ $\otimes \xi$ induites par $\nabla_{T}^{\Lambda\left(T^{*(0,1)} Z\right)},{ }^{0} \nabla^{\Lambda\left(T^{*(0,1)} Z\right)}, \nabla^{\xi}$ définies en $(2.1)$.

Pour $T>0$, on définit la connexion $\nabla_{T}^{\Omega\left(Z, \xi_{\mid Z}\right)}$ sur $\Omega\left(Z, \xi_{\mid Z}\right)$ comme en (2.3) correspondant à $g_{T}^{T Z}, h^{\xi}$. En effet, si $s$ est une section $\mathcal{C}^{\infty} \operatorname{de} \Omega\left(Z, \xi_{\mid Z}\right), U \in T_{\mathbb{R}} S$, on pose

$$
\nabla_{T, U}^{\Omega\left(Z, \xi_{\mid Z}\right)} s=\nabla_{T, U_{3, T}^{H}}^{\Lambda\left(T^{*(0,1)} Z\right) \otimes \xi} s
$$

TOME $127-1999-\mathrm{N}^{\circ} 4$ 
De même, on définit une connexion ${ }^{0} \nabla^{\Omega\left(Z, \xi_{\mid Z}\right)}$ sur $\Omega\left(Z, \xi_{\mid Z}\right)$ par la formule : si $s$ est une section $\mathcal{C}^{\infty}$ de $\Omega\left(Z, \xi_{\mid Z}\right), U \in T_{\mathbb{R}} S$,

$$
{ }^{0} \nabla_{U}^{\Omega\left(Z, \xi_{\mid Z}\right)} s={ }^{0} \nabla_{U_{3, \infty}^{H}}^{\Lambda\left(T^{*(0,1)} Z\right) \otimes \xi} s
$$

Comme dans [BerB, $\S 5]$, on préfère considérer les opérateurs sur $\Lambda\left(T_{\mathbb{R}}^{*} S\right) \widehat{\otimes} E_{0}$ dans le cadre d'une métrique fixée. On va conjuguer la superconnexion $B_{3, u, T}$ définie au paragraphe $4 \mathrm{a}$ ).

DÉfinition 5.7. - Pour $T \geq 1$, soient

$$
A_{u, T}=C_{T} B_{3, u^{2}, T} C_{T}^{-1}, \quad A_{T}=A_{1, T} .
$$

Comme $N_{3, u^{2}, T}$ est invariant par la conjugaison, on a

$$
\begin{aligned}
& \varphi \operatorname{Tr}_{s}\left[N_{3, u^{2}, T} \exp \left(-B_{3, u^{2}, T}^{2}\right)\right]=\varphi \operatorname{Tr}_{s}\left[N_{3, u^{2}, T} \exp \left(-A_{u, T}^{2}\right)\right] \\
& \varphi \operatorname{Tr}_{s}\left[M_{3, u^{2}, T} \exp \left(-B_{3, u^{2}, T}^{2}\right)\right]=\varphi \operatorname{Tr}_{s}\left[C_{T} M_{3, u^{2}, T} C_{T}^{-1} \exp \left(-A_{u, T}^{2}\right)\right]
\end{aligned}
$$

Soit $A_{T}^{(0)}$ (resp. $A_{T}^{(>0)}$ ) la partie de $A_{T}$ de degré 0 (resp. $\left.>0\right)$ dans $\Lambda\left(T_{\mathbb{R}}^{*} S\right)$. D'après [BerB, prop. 5.9], (2.7) et $(5.14)$, on a

$$
\left\{\begin{array}{l}
A_{T}^{(0)}=T D_{T}^{X}+D_{T}^{H} \\
A_{T}^{(>0)}=C_{T}\left(\nabla_{T}^{\Omega\left(Z, \xi_{\mid Z}\right)}-\frac{1}{2 \sqrt{2}} c_{T}\left(T_{3, T}\right)\right) C_{T}^{-1} .
\end{array}\right.
$$

Soit $\mathcal{B}$ la superconnexion sur $E_{0}$

$$
\mathcal{B}={ }^{0} \nabla^{\Omega\left(Z, \xi_{\mid Z}\right)}+D_{\infty}^{H}-\frac{1}{2 \sqrt{2}} c\left(T_{2}\right)
$$

THÉORÈME 5.8. - Quand $T \rightarrow+\infty$, on $a:$

$$
A_{T}=T D^{X}+\mathcal{B}+O\left(\frac{1}{T}\right)
$$

Preuve. - i) D'après (5.2), on a

$$
B_{T}=1+O\left(\frac{1}{T^{2}}\right)
$$

Par (5.7) et (5.19), on a

$$
D_{T}^{H}=D_{\infty}^{H}+O\left(\frac{1}{T^{2}}\right), \quad D_{T}^{X}=D^{X}+O\left(\frac{1}{T^{2}}\right) .
$$

BULLETIN DE LA SOCIÉTÉ MATHÉMATIQUE DE FRANCE 
ii) En utilisant la proposition $1.2,(1.35)$ et $(5.10)$, pour $U \in T_{\mathbb{R}} S$, on a :

$$
\begin{aligned}
& C_{T} \nabla_{T, U}^{\Omega\left(Z, \xi_{\mid Z}\right)} C_{T}^{-1}=B_{T}^{0} \nabla_{U_{3, T}^{H}}^{\Lambda\left(T^{*(0,1)} Z\right) \otimes \xi} B_{T}^{-1} \\
& +\left\langle\overline{\left\langle\left(T^{2}+h\right)^{-1} \nabla_{U_{3, T}^{H}}^{\pi_{1}^{*} T Y^{\prime}} h\right.} \bar{\theta}_{\ell, T}, \theta_{m, T}\right\rangle_{g_{T}^{T Z}} \bar{\theta}^{\ell} \wedge i_{\bar{\theta}_{m}} \\
& +\frac{1}{T}\left\langle A\left(U_{3, T}^{H}\right) \bar{\theta}_{\ell, T}, w_{j}\right\rangle_{g^{T X}} \bar{\theta}^{\ell} \wedge i_{\bar{w}_{j}} \\
& -T\left\langle\left(T^{2}+h\right)^{-1} A^{*}\left(U_{3, T}^{H}\right) \bar{w}_{j}, \theta_{\ell}\right\rangle_{g_{T}^{T Z}} \bar{w}^{j} \wedge i_{\bar{\theta}_{\ell}} \\
& ={ }^{0} \nabla_{U_{3, \infty}^{H}}^{\Lambda\left(T^{*(0,1)} Z\right) \otimes \xi}+O\left(\frac{1}{T}\right) .
\end{aligned}
$$

iii) D'après la proposition 1.2 , le théorème 1.6 et $(5.10)$, on a :

$$
\begin{gathered}
C_{T} c_{T}\left(T_{3, T}\right) C_{T}^{-1}=\sum \frac{1}{2}\left\langle T_{3, T}\left(g_{\alpha, 3, T}^{H}, g_{\beta, 3, T}^{H}\right), T e_{i}\right\rangle_{g_{T}^{T Z}} g^{\alpha} \wedge g^{\beta} \wedge c\left(e_{i}\right) \\
+\sum \frac{1}{2}\left\langle T_{3, T}\left(g_{\alpha, 3, T}^{H}, g_{\beta, 3, T}^{H}\right), f_{\ell, T}\right\rangle_{g_{T}^{T Z}} g^{\alpha} \wedge g^{\beta} \wedge c\left(f_{\ell, 1}^{H}\right) \\
=\sum \frac{1}{2}\left\langle T_{3, \infty}\left(g_{\alpha, 3, \infty}^{H}, g_{\beta, 3, \infty}^{H}\right), f_{\ell, 1}^{H}\right\rangle_{\pi_{1}^{*} g^{T Y}} g^{\alpha} \wedge g^{\beta} \wedge c\left(f_{\ell, 1}^{H}\right)+O\left(\frac{1}{T}\right) .
\end{gathered}
$$

D'après (1.20) et (5.22), on a

$$
C_{T} c_{T}\left(T_{3, T}\right) C_{T}^{-1}=\sum \frac{1}{2}\left\langle T_{2}\left(g_{\alpha, 2}^{H}, g_{\beta, 2}^{H}\right), f_{\ell}\right\rangle_{g^{T Y}} g^{\alpha} \wedge g^{\beta} \wedge c\left(f_{\ell, 1}^{H}\right)+O\left(\frac{1}{T}\right) .
$$

En utilisant (5.19)-(5.23), on a (5.18).

On a bien terminé la preuve du théorème 5.8 .

ThÉORÈme 5.9. - Pour tout $T \in\left[1,+\infty\left[\right.\right.$, l'opérateur $J_{T}^{-1} p_{T} A_{T} p_{T} J_{T}$ est une superconnexion sur $E_{1, \infty}$. Quand $T \rightarrow+\infty$, on $a$ :

$$
J_{T}^{-1} p_{T} A_{T} p_{T} J_{T}=B_{2,1}+O\left(\frac{1}{T}\right) .
$$

Preuve. - Soit $\nabla^{R \pi_{1 *} \xi}$ la connexion holomorphe hermitienne sur $\left(R^{\bullet} \pi_{1 *} \xi, h^{R \pi_{1 *} \xi}\right)$. Soit $\nabla^{\Omega\left(X, \xi_{\mid X}\right)}$ la connexion sur $\Omega\left(X, \xi_{\mid X}\right)$ comme en (2.3) correspondant à $g^{T X}, h^{\xi}$. Alors par [BKö, th. 3.5], on a :

$$
\nabla^{R \pi_{1 *} \xi}=p_{\infty} \nabla^{\Omega\left(X, \xi_{\mid X}\right)} p_{\infty} .
$$

En utilisant [BerB, (5.34)], (5.17) et (5.25), on a :

$$
p_{\infty} \mathcal{B} p_{\infty}=B_{2,1} \text {. }
$$

Par [BerB, (5.47)], on a :

$$
p_{T}=p_{\infty}+O\left(\frac{1}{T^{2}}\right) .
$$

En utilisant le théorème $5.8,(5.9)$ et $(5.26)$, on a $(5.24)$ 


\section{c) La structure de l'opérateur $A_{T}^{2}$ quand $T$ tend vers l'infini.}

Dans la suite, on pose

$$
p=p_{\infty}, \quad p^{\perp}=p_{\infty}^{\perp} .
$$

Maintenant, on va considérer $A_{T}^{2}$ comme un opérateur de $\Lambda\left(T_{\mathbb{R}}^{*} S\right) \widehat{\otimes} E_{0}$ dans $\Lambda\left(T_{\mathbb{R}}^{*} S\right) \widehat{\otimes} E_{0}$. On pose

$$
E_{T}=p A_{T}^{2} p, \quad F_{T}=p A_{T}^{2} p^{\perp}, \quad G_{T}=p^{\perp} A_{T}^{2} p, \quad H_{T}=p^{\perp} A_{T}^{2} p^{\perp} .
$$

Alors on écrit $A_{T}^{2}$ sous forme matricielle correspondant au scindage $E_{0}^{0}=$ $E_{1}^{0} \oplus E_{1}^{0, \perp}$.

$$
A_{T}^{2}=\left(\begin{array}{ll}
E_{T} & F_{T} \\
G_{T} & H_{T}
\end{array}\right)
$$

ThÉORÈme 5.10.- Il existe des opérateurs $E, F, G, H$ tels que, pour $T \rightarrow+\infty$,

$$
\begin{cases}E_{T}=E+O\left(\frac{1}{T}\right), & F_{T}=T F+O(1), \\ G_{T}=T G+O(1), & H_{T}=T^{2} H+O(T) .\end{cases}
$$

Soit

$$
Q_{\infty}=\left[D^{X}, \mathcal{B}\right]
$$

Alors $Q_{\infty}\left(E_{1}^{0}\right) \subset E_{1}^{0, \perp}$, et

$$
\begin{aligned}
Q_{\infty} & =\frac{1}{2} \sum c\left(e_{i}\right) c\left(f_{\ell, 1}^{H}\right)\left[\left(R^{\Lambda\left(T^{*(0,1)} X\right)}+L^{\xi}\right)\left(e_{i}, f_{\ell, 1}^{H}\right)-{ }^{0} \nabla_{T_{1}\left(e_{i}, f_{\ell, 1}^{H}\right)}^{\Lambda\left(T^{*(0,1)} Z\right) \otimes \xi}\right] \\
+ & \frac{1}{\sqrt{2}} \sum c\left(e_{i}\right) g^{\alpha}\left[\left(R^{\Lambda\left(T^{*(0,1)} X\right)}+L^{\xi}\right)\left(e_{i}, g_{\alpha, 3, \infty}^{H}\right)-{ }^{0} \nabla_{T_{1}\left(e_{i}, g_{\alpha, 3, \infty}^{H}\right.}^{\Lambda\left(T^{*(0,1)} Z\right) \otimes \xi}\right]
\end{aligned}
$$

On a aussi

$$
E=p \mathcal{B}^{2} p, \quad F=p Q_{\infty} p^{\perp}, \quad G=p^{\perp} Q_{\infty} p, \quad H=p^{\perp} D^{X, 2} p^{\perp}
$$

Preuve. - D'après le théorème 5.8 , on a

$$
E=p \mathcal{B}^{2} p
$$

D'après le théorème 5.8 , on sait aussi que le coefficient de $T$ (resp. $T^{2}$ ) dans l'asymptotique de $A_{T}^{2}$ quand $T \rightarrow+\infty$ est $\left[D^{X}, \mathcal{B}\right]\left(\right.$ resp. $\left.D^{X, 2}\right)$. 
D'après [BerB, (5.65), (5.66)], on a

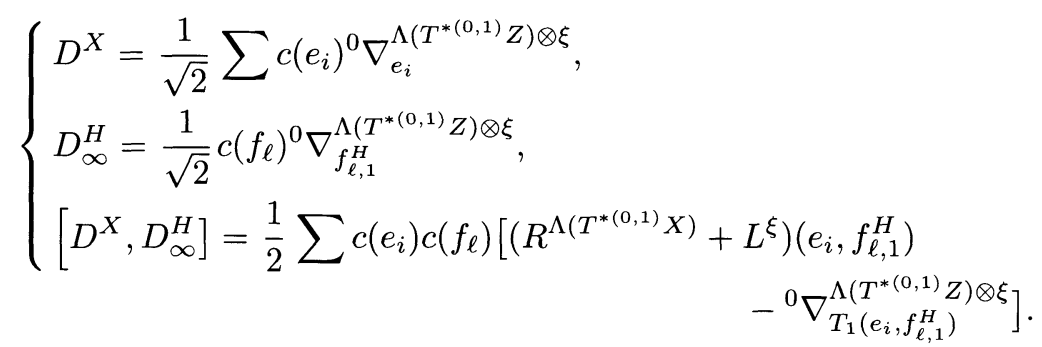

En utilisant (5.13), (5.36), on peut calculer comme dans [BerB, (5.66)],

$$
\begin{aligned}
& {\left[D^{X},{ }^{0} \nabla^{\Omega(Z, \xi \mid Z)}\right]} \\
& =\frac{1}{\sqrt{2}} \sum c\left(e_{i}\right) g^{\alpha}\left[\left(R^{\Lambda\left(T^{*(0,1)} X\right)}+L^{\xi}\right)\left(e_{i}, g_{\alpha, 3, \infty}^{H}\right)-{ }^{0} \nabla_{T_{1}\left(e_{i}, g_{\alpha, 3, \infty}^{H}\right)}^{\Lambda\left(T^{*(0,1)} Z\right) \otimes \xi}\right] .
\end{aligned}
$$

Par (5.32), (5.36) et (5.37), on a (5.33).

Par (5.32), on a aussi

$$
p Q_{\infty} p=0 .
$$

On a montré le théorème 5.10.

\section{d) Le spectre de $A_{u, T}^{2}$.}

Comme on suppose que $S$ est compacte, toutes les estimations qui suivent seront uniformes en $s \in S$. Si $C$ est un opérateur, on note $\operatorname{Sp}(C)$ le spectre de $C$. Soit

$$
\begin{aligned}
R_{u, T}=C_{T}\left(\nabla_{T}^{\Omega(Z, \xi \mid Z)}-\right. & \left.\frac{c_{T}\left(T_{3, T}\right)}{2 \sqrt{2} u}\right)^{2} C_{T}^{-1} \\
& +u\left[C_{T}\left(\nabla_{T}^{\Omega(Z, \xi \mid Z)}-\frac{c_{T}\left(T_{3, T}\right)}{2 \sqrt{2} u}\right) C_{T}^{-1}, A_{T}^{(0)}\right] .
\end{aligned}
$$

Alors

$$
A_{u, T}^{2}=u^{2} A_{T}^{(0), 2}+R_{u, T} .
$$

Par [B1, th. 2.5], $R_{u, T}$ est la somme de formes de degré strictement positif dans $\Lambda\left(T_{\mathbb{R}}^{*} S\right)$, à valeur dans les opérateurs différentiels d'ordre 1 le long de la fibre $Z$.

Les opérateurs $A_{u, T}^{2}$ et $A_{T}^{(0), 2}$ sont des opérateurs non bornés agissant sur $\Lambda\left(T_{\mathbb{R}}^{*} S\right) \widehat{\otimes} E_{0}$, dont le domaine est $\Lambda\left(T_{\mathbb{R}}^{*} S\right) \widehat{\otimes} E_{0}^{2}$.

ThÉORÈme 5.11. - Il existe $c_{1}>0, T_{0} \geq 1$ tels que, pour tout $T \geq T_{0}$,

$$
\operatorname{Sp}\left(A_{T}^{(0), 2}\right) \cap\left\{\lambda \in \mathbb{R}_{+}^{*}, \lambda \leq c_{1}\right\} \subset\{0\}
$$

Preuve. - En utilisant (3.4), [BerB, $\S 6$ (d)], on a (5.41). 
D'après [B4, prop. 9.2], on a :

Proposition 5.12. - Pour tout $u>0, T>0$,

$$
\operatorname{Sp}\left(A_{u, T}^{2}\right)=\operatorname{Sp}\left(u^{2} A_{T}^{(0), 2}\right)
$$

Soit $\Gamma^{\prime}=\delta \cup \Delta$ le contour suivant dans $\mathbb{C}$ :

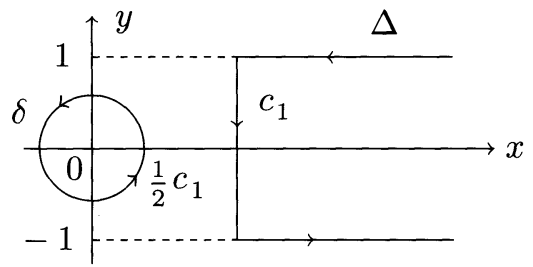

Par le théorème 5.11 et la proposition 5.12 , il est clair que pour $u \geq 1$ et $T \geq T_{0}$, on a

$$
\left\{\begin{array}{l}
\exp \left(-A_{u, T}^{2}\right)=\frac{1}{2 \pi i} \int_{\Delta} \frac{\exp \left(-u^{2} \lambda\right)}{\lambda-u^{-2} A_{u, T}^{2}} \mathrm{~d} \lambda+\frac{1}{2 \pi i} \int_{\delta} \frac{\exp (-\lambda)}{\lambda-A_{u, T}^{2}} \mathrm{~d} \lambda \\
\exp \left(-u^{2} A_{T}^{2}\right)=\frac{1}{2 \pi i} \int_{\Gamma^{\prime}} \frac{\exp \left(-u^{2} \lambda\right)}{\lambda-A_{T}^{2}} \mathrm{~d} \lambda
\end{array}\right.
$$

e) Deux résultats intermédiaires.

Dans la suite, $u_{0}$ est une constante positive fixée.

Pour $u>0$, soit $\psi_{u}: \Lambda\left(T_{\mathbb{R}}^{*} S\right) \rightarrow \Lambda\left(T_{\mathbb{R}}^{*} S\right)$ l'application

$$
\alpha \in \Lambda\left(T_{\mathbb{R}}^{*} S\right) \longrightarrow u^{-\operatorname{deg} \alpha} \alpha \in \Lambda\left(T_{\mathbb{R}}^{*} S\right)
$$

Alors $\psi_{u}$ agit comme $\psi_{u} \widehat{\otimes} 1$ sur $\Lambda\left(T_{\mathbb{R}}^{*} S\right) \widehat{\otimes} E_{0}$.

Proposition 5.13. - Pour $u>0$ et $T>0$, on $a:$

$$
\begin{cases}A_{u, T}=u \psi_{u} A_{T} \psi_{u}^{-1}, & B_{2, u^{2}}=u \psi_{u} B_{2,1} \psi_{u}^{-1} \\ N_{3, u^{2}, T}=\psi_{u} N_{3,1, T} \psi_{u}^{-1}, & N_{2, u^{2}}=\psi_{u} N_{2,1} \psi_{u}^{-1} \\ M_{3, u^{2}, T}=\psi_{u} M_{3,1, T} \psi_{u}^{-1} & \end{cases}
$$

Preuve. - C'est évident.

BULLETIN DE LA SOCIÉTÉ MATHÉMATIQUE DE FRANCE 
Proposition 5.14. - Pour $u>0, T>0$, on $a:$

$$
\left\{\begin{aligned}
\operatorname{Tr}_{s}\left[C_{T} M_{3, u^{2}, T} C_{T}^{-1} \exp \left(-A_{u, T}^{2}\right)\right] & \\
& =\psi_{u} \operatorname{Tr}_{s}\left[C_{T} M_{3,1, T} C_{T}^{-1} \exp \left(-u^{2} A_{T}^{2}\right)\right] \\
\operatorname{Tr}_{s}\left[N_{3, u^{2}, T}\right. & \left.\frac{1}{2 \pi i} \int_{\Delta} \frac{\exp \left(-u^{2} \lambda\right)}{\lambda-u^{-2} A_{u, T}^{2}} \mathrm{~d} \lambda\right] \\
& =\psi_{u} \operatorname{Tr}_{s}\left[N_{3,1, T} \frac{1}{2 \pi i} \int_{\Delta} \frac{\exp \left(-u^{2} \lambda\right)}{\lambda-A_{T}^{2}} \mathrm{~d} \lambda\right]
\end{aligned}\right.
$$

et pour $u>0$, on $a$ :

$$
\begin{aligned}
\operatorname{Tr}_{s}\left[N_{2, u^{2}} \frac{1}{2 \pi i} \int_{\Delta} \frac{\exp \left(-u^{2} \lambda\right)}{\lambda-u^{-2} B_{2, u^{2}}^{2}} \mathrm{~d} \lambda\right] \\
\quad=\psi_{u} \operatorname{Tr}_{s}\left[N_{2,1} \frac{1}{2 \pi i} \int_{\Delta} \frac{\exp \left(-u^{2} \lambda\right)}{\lambda-B_{2,1}^{2}} \mathrm{~d} \lambda\right] .
\end{aligned}
$$

Preuve. - C'est une conséquence directe de la proposition 5.13.

ThÉorème 5.15. - Il existe $\delta \in] 0,1]$ et $C>0$ tels que, pour $u \geq u_{0}$ et $T \geq T_{0}$,

$$
\begin{aligned}
& \mid \operatorname{Tr}_{s}\left[N_{3,1, T} \frac{1}{2 \pi i} \int_{\Delta} \frac{\exp \left(-u^{2} \lambda\right)}{\lambda-A_{T}^{2}} \mathrm{~d} \lambda\right] \\
& \quad-\operatorname{Tr}_{s}\left[N_{2,1} \frac{1}{2 \pi i} \int_{\Delta} \frac{\exp \left(-u^{2} \lambda\right)}{\lambda-B_{2,1}^{2}} \mathrm{~d} \lambda\right] \mid \leq \frac{C}{T^{\delta}} .
\end{aligned}
$$

Il existe $c>0$ et $C>0$ tels que, pour $u \geq u_{0}$ et $T \geq T_{0}$,

$$
\left|\operatorname{Tr}_{s}\left[N_{3,1, T} \frac{1}{2 \pi i} \int_{\Delta} \frac{\exp \left(-u^{2} \lambda\right)}{\lambda-A_{T}^{2}} \mathrm{~d} \lambda\right]\right| \leq c \exp \left(-C u^{2}\right) .
$$

Théorème 5.16. - Il existe $\delta \in] 0,1]$ et $c>0$ tels que, pour $u \geq u_{0}, T \geq T_{0}$,

$$
\begin{aligned}
\mid \operatorname{Tr}_{s}\left[N_{3, u^{2}, T} \frac{1}{2 \pi i} \int_{\delta} \frac{\exp (-\lambda)}{\lambda-A_{u, T}^{2}} \mathrm{~d} \lambda\right] & \\
-\operatorname{Tr}_{s} & {\left[N_{2, u^{2}} \frac{1}{2 \pi i} \int_{\delta} \frac{\exp (-\lambda)}{\lambda-B_{2, u^{2}}^{2}} \mathrm{~d} \lambda\right] \mid \leq \frac{C}{T^{\delta}} . }
\end{aligned}
$$

Il existe $C>0$ tel que, pour $u \geq u_{0}, T \geq T_{0}$,

$$
\left|\operatorname{Tr}_{s}\left[N_{3, u^{2}, T} \frac{1}{2 \pi i} \int_{\delta} \frac{\exp (-\lambda)}{\lambda-A_{u, T}^{2}} \mathrm{~d} \lambda\right]\right| \leq \frac{C}{u} .
$$

Le reste de ce paragraphe est consacré à la preuve des théorèmes 5.15 et 5.16 . TOME $127-1999-\mathrm{N}^{\circ} 4$ 
Remarque 5.17. - Comme dans [B4, rem.9.7], les équations (4.11), et le théorème 4.6 peuvent être déduits des théorèmes 5.15 et 5.16 .

f) Propriétés de régularité de la résolvante de $\boldsymbol{A}_{T}^{\mathbf{2}}$.

Soit $e_{1}, \cdots, e_{2 n_{0}}$ (resp. $f_{1}, \cdots, f_{2 m_{0}}$ ) une base orthonormée locale $\mathcal{C}^{\infty}$ de $T_{\mathbb{R}} X$ (resp. $T_{\mathbb{R}} Y$ ). Pour $s, s^{\prime} \in E_{0}$, on pose :

$$
|s|_{0}=\|s\|_{E_{0}^{0}}, \quad\left\langle s, s^{\prime}\right\rangle_{0}=\left\langle s, s^{\prime}\right\rangle_{E_{0}^{0}}
$$

DÉfinition 5.18. - Pour $T>1$ et $s \in E_{0}$, on pose :

$$
\begin{aligned}
|s|_{T, 1}^{2}=\left|p_{T} s\right|^{2}+T^{2}\left|p_{T}^{\perp} s\right|_{0}^{2}+\sum_{\ell}\left|{ }^{0} \nabla_{f_{\ell, 1}^{H}}^{\Lambda\left(T^{*(0,1)} Z\right) \otimes \xi} s\right|_{0}^{2} & \\
& +T^{2} \sum_{i}\left|{ }^{0} \nabla_{e_{i}}^{\Lambda\left(T^{*(0,1)} Z\right) \otimes \xi} p_{T}^{\perp} s\right|_{0}^{2} .
\end{aligned}
$$

Alors (5.53) définit une norme sur $E_{0}^{1}$, et $\left(E_{0}^{1},||_{T, 1}\right)$ se plonge continûment dans $\left(E_{0}^{0},||_{0}\right)$. On identifie $E_{0}^{0}$ à son antidual par \langle\rangle$_{0}$. Alors on peut identifier $E_{0}^{-1}$ à l'antidual de $E_{0}^{1}$. Soit ||$_{T,-1}$ la norme sur $E_{0}^{-1}$ associée à ||$_{T, 1}$. Alors on a les inclusions continues denses suivantes dont les normes sont inférieures à 1 ,

$$
E_{0}^{1} \longrightarrow E_{0}^{0} \longrightarrow E_{0}^{-1} \text {. }
$$

Soit $g^{T S}$ une métrique sur $T S$. Alors la définition de $|s|_{0},|s|_{T, 1}$ se prolonge naturellement à $\Lambda\left(T_{\mathbb{R}}^{*} S\right) \widehat{\otimes} E_{0}$. Soit

$$
R_{T}=R_{1, T}=\left[A_{T}^{(>0)}, A_{T}^{(0)}\right]+A_{T}^{(>0), 2} .
$$

ThÉORÈme 5.19. - Il existe $C_{1}, C_{2}, C_{3}>0, T_{0}>0$ tels que, pour $T \geq T_{0}$, $s, s^{\prime} \in \Lambda\left(T_{\mathbb{R}}^{*} S\right) \widehat{\otimes} E_{0}$,

$$
\left\{\begin{array}{l}
\left|A_{T}^{(0)} s\right|_{0}^{2} \geq C_{1}|s|_{T, 1}^{2}-C_{2}|s|_{0}^{2} \\
\left|\left\langle A_{T}^{(0)} s, A_{T}^{(0)} s^{\prime}\right\rangle_{0}\right| \leq C_{3}|s|_{T, 1}\left|s^{\prime}\right|_{T, 1} \\
\left|\left\langle R_{T} s, s^{\prime}\right\rangle_{0}\right| \leq C_{3}\left(|s|_{T, 1}\left|s^{\prime}\right|_{0}+|s|_{0}\left|s^{\prime}\right|_{T, 1}\right)
\end{array}\right.
$$

Preuve. - D'après la preuve de [BerB, th. 5.19], il existe $C>0, T_{0}>0$ tels que, pour $T \geq T_{0}, s \in E_{1, T}^{1, \perp}$,

$$
\left|A_{T}^{(0)} s\right|_{0}^{2} \geq C\left[\|s\|_{E_{0}^{1}}^{2}+T^{2}\left(\left|D_{T}^{X} s\right|^{2}+|s|_{0}^{2}\right)\right] .
$$

En utilisant [BerB, th. 5.17, et 5.18], (5.53), on a la première inégalité de (5.56).

La deuxième inégalité de (5.56) est triviale. 
Par (5.16), pour $s, s^{\prime} \in \Lambda\left(T_{\mathbb{R}}^{*} S\right) \widehat{\otimes} E_{0}$, on a

$$
\begin{array}{r}
\left|\left\langle R_{T} s, s^{\prime}\right\rangle_{0}\right| \\
\leq c\left(|s|_{T, 1}\left|s^{\prime}\right|_{0}+|s|_{0}\left|s^{\prime}\right|_{T, 1}\right)+\left|\left\langle\left[A_{T}^{(>0)}, A_{T}^{(0)}\right] p_{T} s, p_{T} s^{\prime}\right\rangle_{0}\right| .
\end{array}
$$

Mais

$$
\begin{aligned}
\left|\left\langle\left[A_{T}^{(>0)}, A_{T}^{(0)}\right] p_{T} s, p_{T} s^{\prime}\right\rangle_{0}\right| & =\left|\left\langle\left[D_{T}^{H}, A_{T}^{(>0)}\right] p_{T} s, p_{T} s^{\prime}\right\rangle_{0}\right| \\
& \leq c|s|_{T, 1}\left|s^{\prime}\right|_{0} .
\end{aligned}
$$

D'après (5.58), (5.59), on a la troisième inégalité de (5.56). On a bien terminé la preuve du théorème.

Comme $S$ est compacte, il existe $U_{1}, \cdots, U_{m}$ (resp. $U_{1}^{\prime}, \cdots, U_{m^{\prime}}^{\prime}$ ) une famille de sections $\mathcal{C}^{\infty}$ de $T_{\mathbb{R}} Y$ (resp. $\left.T_{\mathbb{R}} X\right)$ telle que, pour tout $y \in V$, (resp. $x \in W$ ), $U_{1}(y), \cdots, U_{m}(y)$ (resp. $\left.U_{1}^{\prime}(x), \cdots, U_{m^{\prime}}^{\prime}(x)\right)$ engendrent $\left(T_{\mathbb{R}} Y\right)_{y}\left(\operatorname{resp} .\left(T_{\mathbb{R}} X\right)_{x}\right)$.

DÉfinition 5.20. - Pour $T>0$, soit $\mathcal{D}_{T}$ la famille d'opérateurs agissant sur $E_{0}$

$$
\begin{gathered}
\mathcal{D}_{T}=\left\{p_{T}^{0} \nabla_{U_{\ell, 1}^{H}}^{\Lambda\left(T^{*(0,1)} Z\right) \otimes \xi} p_{T}, p_{T}^{\perp 0} \nabla_{U_{\ell, 1}^{H}}^{\Lambda\left(T^{*(0,1)} Z\right) \otimes \xi} p_{T}^{\perp}\right. \\
\left.p_{T}^{\perp 0} \nabla_{U_{i}^{\prime}}^{\Lambda\left(T^{*(0,1)} Z\right) \otimes \xi} p_{T}^{\perp}\right\}
\end{gathered}
$$

ThÉorème 5.21. - Pour tout $k \in \mathbb{N}$, il existe $C_{k}>0$ tel que, pour $T \geq 1$, $Q_{1}, \ldots, Q_{k} \in \mathcal{D}_{T}$ et $s, s^{\prime} \in \Lambda\left(T_{\mathbb{R}}^{*} S\right) \widehat{\otimes} E_{0}$, on $a:$

$$
\left|\left\langle\left[Q_{1},\left[Q_{2}, \cdots\left[Q_{k}, A_{T}^{2}\right], \cdots\right]\right] s, s^{\prime}\right\rangle_{0}\right| \leq\left. C_{k}|s|_{T, 1}|\cdot| s^{\prime}\right|_{T, 1} \text {. }
$$

Preuve. - Comme

$$
\left[{ }^{0} \nabla_{U_{\ell, 1}^{H}}^{\Lambda\left(T^{*(0,1)} Z\right) \otimes \xi}, p_{T}\right],{ }^{0} \nabla_{U_{i}^{\prime}}^{\Lambda\left(T^{*(0,1)} Z\right) \otimes \xi} p_{T}, p_{T}{ }^{0} \nabla_{U_{i}^{\prime}}^{\Lambda\left(T^{*(0,1)} Z\right) \otimes \xi}
$$

sont des opérateurs dont les restrictions aux fibres $X_{b}$ (avec $b \in V$ ) ont des noyaux $\mathcal{C}^{\infty}$ uniformément bornés pour $x, x^{\prime} \in X_{b}, b \in V$ et $T \geq 1$, en procédant comme dans la preuve de [B4, th. 9.17], on obtient le théorème.

Soit

$$
F_{u}\left(A_{T}^{2}\right)=\frac{1}{2 \pi i} \int_{\Delta} \mathrm{e}^{-u^{2} \lambda}\left(\lambda-A_{T}^{2}\right)^{-1} \mathrm{~d} \lambda
$$

Soient $P_{u, T}\left(x, x^{\prime}\right), F_{u}\left(A_{T}^{2}\right)\left(x, x^{\prime}\right)$ (avec $x, x^{\prime} \in Z_{s}$ ) les noyaux $\mathcal{C}^{\infty}$ des opérateurs $\exp \left(-u^{2} A_{T}^{2}\right)$ et $F_{u}\left(A_{T}^{2}\right)$ relativement à $d v_{Z}\left(x^{\prime}\right) /(2 \pi)^{\operatorname{dim} Z}$.

$$
\text { TOME } 127-1999-\mathrm{N}^{\circ} 4
$$


g) La borne uniforme des noyaux $\operatorname{de} \exp \left(-u^{2} A_{T}^{2}\right)$ et $F_{u}\left(A_{T}^{2}\right)$.

THÉORÈME 5.22 .

(i) Pour $m \in \mathbb{N}$ et $0<u_{1}<u_{2}$ fixés, il existe $C>0$ tel que, pour $x, x^{\prime} \in Z_{s}$, $u \in\left[u_{1}, u_{2}\right], T \geq T_{0}$,

$$
\sup _{|\alpha|,\left|\alpha^{\prime}\right| \leq m}\left|\frac{\partial^{|\alpha|+\left|\alpha^{\prime}\right|}}{\partial x^{\alpha} \partial x^{\prime \alpha^{\prime}}} P_{u, T}\left(x, x^{\prime}\right)\right| \leq C .
$$

(ii) Pour $m \in \mathbb{N}$, il existe $c>0$ et $C^{\prime}>0$ tels que, pour $x, x^{\prime} \in Z_{s}, u \geq u_{0}$, $T \geq T_{0}$,

$$
\sup _{|\alpha|,\left|\alpha^{\prime}\right| \leq m}\left|\frac{\partial^{|\alpha|+\left|\alpha^{\prime}\right|}}{\partial x^{\alpha} \partial x^{\prime \alpha^{\prime}}} F_{u}\left(A_{T}^{2}\right)\left(x, x^{\prime}\right)\right| \leq c \exp \left(-C^{\prime} u^{2}\right) .
$$

Preuve. - En utilisant les théorèmes 5.19 et 5.21, comme

$$
\left[{ }^{0} \nabla_{U_{\ell, 1}^{H}}^{\Lambda\left(T^{*(0,1)} Z\right) \otimes \xi}, p_{T}\right],{ }^{0} \nabla_{U_{i}^{\prime}}^{\Lambda\left(T^{*(0,1)} Z\right) \otimes \xi} p_{T}, p_{T}^{0} \nabla_{U_{i}^{\prime}}^{\Lambda\left(T^{*(0,1)} Z\right) \otimes \xi}
$$

sont des opérateurs dont les restrictions aux fibres $X_{b}$ (avec $b \in V$ ) ont des noyaux $\mathcal{C}^{\infty}$ uniformément bornées pour $x, x^{\prime} \in X_{b}, b \in V, T \geq 1$, en procédant comme dans la preuve de [B4, th. 9.20], on a le théorème 5.22 .

h) L'asymptotique de l'opérateur $F_{u}\left(A_{T}^{2}\right)$ quand $T$ tend vers l'infini.

DÉfinition 5.23. - Soit $\Xi$ l'opérateur différentiel d'ordre 2 agissant sur $\Lambda\left(T_{\mathbb{R}}^{*} S\right) \widehat{\otimes} E_{1}$,

$$
\Xi=p\left(E-F H^{-1} G\right) p
$$

ThÉORÈme 5.24. - On a l'identité

$$
B_{2,1}^{2}=\Xi
$$

Preuve. - D'après (5.26), (5.32) et (5.34), on a (5.66).

Pour $A \in L\left(E_{0}^{0}, E_{0}^{0}\right)$, on note $\|A\|^{0,0}$ la norme de $A$ relativement à ||$_{0}$. 
THÉORÈME 5.25 .

(i) Pour $0<u_{1}<u_{2}$ fixés, il existe $C>0$ tel que, pour $u \in\left[u_{1}, u_{2}\right], T \geq T_{0}$,

$$
\left\|\exp \left(-u^{2} A_{T}^{2}\right)-p_{T} J_{T} \exp \left(-u^{2} B_{2,1}^{2}\right) J_{T}^{-1} p_{T}\right\|^{0,0} \leq \frac{c}{T^{1 / 4}} .
$$

(ii) Il existe $c>0, C>0$ tels que, pour $u \geq u_{0}, T \geq T_{0}$,

$$
\left\|F_{u}\left(A_{T}^{2}\right)-p_{T} J_{T} F_{u}\left(B_{2,1}^{2}\right) J_{T}^{-1} p_{T}\right\|^{0,0} \leq \frac{c}{T^{1 / 4}} \exp \left(-C u^{2}\right)
$$

Preuve. - En utilisant les théorèmes 5.10 et 5.24, en procédant comme dans la preuve de [BL, th. 13.42], on a le théorème 5.25.

i) Preuves du théorème 5.15 et de (4.12).

Par (4.1), (4.3) et (5.10) (on peut aussi se référer au $\S 7$ ), pour $u>0$ fixé, on a

$$
\left|C_{T} M_{3, u^{2}, T} C_{T}^{-1}-\frac{2}{T}\left(N_{X}-\operatorname{dim} X\right)\right| \leq \frac{C}{T^{3}}
$$

En utilisant (5.15), la proposition 5.14, les théorèmes 5.22 et 5.25 , et en procédant comme en [BL, $\S 11(\mathrm{p})$ et $\S 13(\mathrm{q})]$, on a le théorème 5.15 et (4.12).

\section{j) Preuve du théorème 5.16.}

DÉfinition 5.26. - Pour $a \in \mathbb{C}^{*}, b, c \in \mathbb{C}$ et $T \geq 1$, on pose

$$
\begin{aligned}
\mathcal{G}_{a, b, c, T}=\frac{1}{a^{2}} A_{T}^{(0), 2}+ & C_{T}\left(\nabla_{T}^{\Omega(Z, \xi \mid Z)}-\frac{b}{2 \sqrt{2} u} c_{T}\left(T_{3, T}\right)\right)^{2} C_{T}^{-1} \\
& +u\left[C_{T}\left(c \nabla_{T}^{\Omega(Z, \xi \mid Z)}-\frac{c_{T}\left(T_{3, T}\right)}{2 \sqrt{2} u}\right) C_{T}^{-1}, A_{T}^{(0)}\right]
\end{aligned}
$$

Ces opérateurs sont les analogues des opérateurs définis dans [B4, (9.151)]. En procédant comme en $[\mathrm{B} 4, \S 9(\mathrm{~m})]$, on a aussi des résultats analogues à $[\mathrm{B} 4$, th. 9.29 et 9.30$]$ pour l'opérateur $\mathcal{G}_{a, b, c, T}$.

Évidemment

$$
A_{u, T}^{2}=\mathcal{G}_{1 / u, 1 / u, u, T}
$$

En procédant comme en $[\mathrm{B} 4, \S 9(\mathrm{~m})$ et $(\mathrm{n})]$, on a le théorème 5.16 .

TOME $127-1999-\mathrm{N}^{\circ} 4$ 


\section{Preuve de la proposition 4.7}

On utilise les mêmes notations qu'aux $\S \S 4$ b) et 5 a). On suppose aussi que $\xi$ vérifie l'hypothèse (3.4).

On rappelle que $h \in \operatorname{End}\left(T^{H} Z\right)$ est défini à la définition 1.8.

Pour $\alpha, \alpha^{\prime} \in E_{2}=H\left(Z, \xi_{\mid Z}\right)=H^{0}\left(Z, \xi_{\mid Z}\right)$, on a :

$$
\left\{\begin{array}{l}
\left\langle\alpha, \alpha^{\prime}\right\rangle_{h^{E_{2}}}=\frac{1}{(2 \pi)^{\operatorname{dim} Z}} \int_{Z}\left\langle\alpha, \alpha^{\prime}\right\rangle_{h^{\xi}} \mathrm{d} v_{Y} \mathrm{~d} v_{X} \\
\left\langle\alpha, \alpha^{\prime}\right\rangle_{h_{T}^{H\left(Z, \xi_{\mid Z}\right)}}=\frac{1}{(2 \pi)^{\operatorname{dim} Z}} T^{-2 \operatorname{dim} X} \int_{Z} \operatorname{det}\left(1+\frac{h}{T^{2}}\right)\left\langle\alpha, \alpha^{\prime}\right\rangle_{h^{\xi}} \mathrm{d} v_{Y} \mathrm{~d} v_{X} .
\end{array}\right.
$$

Soit $h_{T}^{\prime}=T^{2 \operatorname{dim} X} h_{T}^{H\left(Z, \xi_{\mid Z}\right)}$ la métrique sur $H\left(Z, \xi_{\mid Z}\right)$. Alors par (6.1), on sait que

$$
h_{T}^{\prime}=h^{E_{2}}+O\left(\frac{1}{T^{2}}\right)
$$

En utilisant (6.1), on a

$$
\nabla_{T}^{H\left(Z, \xi_{\mid Z}\right)}=\nabla^{E_{2}}+O\left(\frac{1}{T^{2}}\right)
$$

$\operatorname{Par}[\mathrm{BKö},(3.31)]$, on a

$$
Q_{T}^{H\left(Z, \xi_{\mid Z}\right)}=-\left(h_{T}^{\prime}\right)^{-1} \frac{\partial}{\partial T} h_{T}^{\prime}+\frac{2 \operatorname{dim} X}{T} .
$$

En utilisant (6.3) et (6.4), on peut donc poser

$$
\begin{aligned}
& H\left(H\left(Z, \xi_{\mid Z}\right), h^{E_{2}}, h^{H\left(Z, \xi_{\mid Z}\right)}\right) \\
& \quad=\int_{1}^{+\infty}\left\{\varphi \operatorname{Tr}_{s}\left[Q_{T}^{H\left(Z, \xi_{\mid Z}\right)} \exp \left(-\nabla_{T}^{H\left(Z, \xi_{\mid Z}\right), 2}\right)\right]\right. \\
& \left.\quad+\varphi \operatorname{Tr}_{s}\left[\frac{2\left(N_{X}-\operatorname{dim} X\right)}{T} \exp \left(-\nabla^{E_{2}, 2}\right)\right]\right\} \mathrm{d} T
\end{aligned}
$$

D'après [BGS1, cor. 1.30 et rem. 1.31], (6.2), (6.5), on a dans $P^{S} / P^{S, 0}$

$$
H\left(H\left(Z, \xi_{\mid Z}\right), h^{E_{2}}, h^{H\left(Z, \xi_{\mid Z}\right)}\right)=-\widetilde{\mathrm{ch}}\left(E_{2}, H\left(Z, \xi_{\mid Z}\right), h^{E_{2}}, h^{H\left(Z, \xi_{\mid Z}\right)}\right) .
$$

Par (6.5) et (6.6), on a bien terminé la preuve de la proposition 4.7. 


\section{Preuve du théorème 4.8}

Le but de ce paragraphe est de montrer le théorème 4.8, relatif à l'asymptotique quand $\varepsilon \rightarrow 0$, de certaines supertraces où apparaît l'opérateur $B_{3, \varepsilon^{2}, T / \varepsilon}$. Ce paragraphe est une extension de $[\mathrm{BerB}, \S 7]$, dans laquelle le théorème 4.8 est établi quand $S$ est un point.

En remplaçant le théorème d'indice local par le théorème d'indice local relatif, on applique les techniques de [BerB, §7], à notre situation.

Ce paragraphe est organisé de la façon suivante. Dans a), on remplace l'opérateur $B_{3, \varepsilon^{2}, T / \varepsilon}$ par un opérateur conjugué $L_{3, \varepsilon, T}^{0}$ et on établit un formule de Lichnerowicz pour $L_{3, \varepsilon, T}^{0}$. Dans b), on fait un changement de coordonnées locales sur $Y_{s_{0}}$ et un changement d'échelle [Ge] sur l'algèbre de Clifford [BeGeV]. Dans c), on calcule l'asymptotique des opérateurs $L_{3, \varepsilon, T}^{3}$ et $M_{3, \varepsilon, T}^{3}$ quand $\varepsilon$ tend vers zéro. Dans d), on conjugue l'opérateur $L_{3,0, T}^{3}$ pour faire apparaître la superconnexion de Levi-Civita. Dans e), on montre le théorème 4.8.

On utilise les mêmes notations qu'aux paragraphes $1 \mathrm{~b}$ ), $4,5 \mathrm{a}$ ) et $5 \mathrm{~b}$ ).

\section{a) Une formule de Lichnerowicz.}

Dans cette partie, on va utiliser souvent les notations du $\S 5 \mathrm{~b}$ ).

On rappelle qu'on note $e_{i}, f_{\ell}, \theta_{\ell}$ des bases orthonormées de $\left(T_{\mathbb{R}} X, g^{T_{\mathbb{R}}} X\right),\left(T_{\mathbb{R}} Y\right.$, $\left.g^{T_{\mathrm{R}} Y}\right),\left(T Y, g^{T Y}\right)$, et qu'on note $e^{i}, f^{i}, \theta^{\ell}$ les bases duales associées. Soit $g_{\alpha}$ une base de $T_{\mathbb{R}} S$; soit $g^{\alpha}$ la base duale associée de $T_{\mathbb{R}}^{*} S$.

On va utiliser la notation suivante : soit $C$ une section $\mathcal{C}^{\infty}$ de $T_{\mathbb{R}}^{*} X \widehat{\otimes}$ $\operatorname{End}\left(\Lambda\left(T^{*(0,1)} X\right) \otimes \xi\right) ;$ alors

$$
\begin{aligned}
\left(\nabla_{e_{i}}^{\Lambda\left(T^{*(0,1)} X\right) \otimes \xi}+C\left(e_{i}\right)\right)^{2}=\sum_{i} & \left(\nabla_{e_{i}}^{\Lambda\left(T^{*(0,1)} X\right) \otimes \xi}+C\left(e_{i}\right)\right)^{2} \\
& -\nabla_{\Sigma_{i} \nabla_{e_{i}}^{T X} e_{i}}^{\Lambda\left(T^{*(0,1)} X\right) \otimes \xi}-C\left(\Sigma_{i} \nabla_{e_{i}}^{T X} e_{i}\right) .
\end{aligned}
$$

On pose

$$
\left\{\begin{array}{c}
M_{3, \varepsilon, T}^{0}=\frac{1}{\varepsilon}\left(\frac{T}{\varepsilon}\right)^{N_{X}} M_{3, \varepsilon^{2}, T / \varepsilon}\left(\frac{T}{\varepsilon}\right)^{-N_{X}}, \\
L_{3, \varepsilon, T}^{0}=\left(\frac{T}{\varepsilon}\right)^{N_{X}} B_{3, \varepsilon^{2}, T / \varepsilon}^{2}\left(\frac{T}{\varepsilon}\right)^{-N_{X}} .
\end{array}\right.
$$

Alors on a :

$$
\operatorname{Tr}_{s}\left[\frac{1}{\varepsilon} M_{3, \varepsilon^{2}, T / \varepsilon} \exp \left(-B_{3, \varepsilon^{2}, T / \varepsilon}^{2}\right)\right]=\operatorname{Tr}_{s}\left[M_{3, \varepsilon, T}^{0} \exp \left(-L_{3, \varepsilon, T}^{0}\right)\right]
$$

TOME $127-1999-\mathrm{N}^{\circ} 4$ 
En utilisant [BerB, (7.5) et (7.36)], (4.1), (4.3), (5.10) et (7.2), on a

$$
\begin{aligned}
& M_{3, \varepsilon, T}^{0}=\frac{i}{T^{3}} \widetilde{\omega}^{W}\left(g_{\alpha, 3, T / \varepsilon}^{H}, g_{\beta, 3, T / \varepsilon}^{H}\right) g^{\alpha} \wedge g^{\beta} \\
+ & \frac{\sqrt{2} i}{T^{2}} \widetilde{\omega}^{W}\left(g_{\alpha, 3, T / \varepsilon}^{H}, e_{i}\right) g^{\alpha} c\left(e_{i}\right)+\frac{\sqrt{2} i \varepsilon}{T^{2}} \widetilde{\omega}^{W}\left(g_{\alpha, 3, T / \varepsilon}^{H}, f_{\ell, T / \varepsilon}\right) g^{\alpha} c_{T / \varepsilon}\left(f_{\ell, T / \varepsilon}\right) \\
+ & \frac{2}{T}\left(N_{X}-\operatorname{dim} X\right)-\frac{2 \varepsilon^{2}}{T^{3}}\left\langle h\left(1+\frac{\varepsilon^{2}}{T^{2}} h\right)^{-1} \bar{\theta}_{\ell}, \theta_{m}\right\rangle_{g^{T Y}} i_{\bar{\theta}_{m}} \wedge \bar{\theta}^{\ell} .
\end{aligned}
$$

Soit

$$
L_{3, T}^{\prime \xi}=L^{\xi}+\frac{1}{2} \operatorname{Tr} R_{T}^{T Z} .
$$

En utilisant le théorème 1.4 , on sait que, quand $T \rightarrow+\infty$, on a :

$$
\operatorname{Tr} R_{T}^{T Z}=\operatorname{Tr} R^{T X}+\operatorname{Tr} R^{T Y}+O\left(\frac{1}{T^{2}}\right)
$$

Soit $K_{1 / \varepsilon}^{Z}$ la courbure scalaire de $\left(T Z, \tilde{g}^{T Z}+\frac{1}{\varepsilon^{2}} \pi_{1}^{*} g^{T Y}\right)$. Soit ${ }^{\prime} \nabla_{T}^{\Lambda\left(T^{*(0,1)} Z\right) \otimes \xi}$ la connexion sur $\Lambda\left(T^{*(0,1)} Z\right) \otimes \xi$ :

$$
{ }^{\prime} \nabla_{T}^{\Lambda\left(T^{*(0,1)} Z\right) \otimes \xi}=T^{N_{X}} \nabla_{T}^{\Lambda\left(T^{*(0,1)} Z\right) \otimes \xi} T^{-N_{X}} .
$$

Pour simplifier, dans l'équation suivante, on identifie $g_{\alpha}, f_{\ell}$ aux relèvements $g_{\alpha, 3, T / \varepsilon}^{H}, f_{\ell, T / \varepsilon}$. En utilisant la formule de Lichnerowicz [B1, th. 3.6], on a

$$
\begin{aligned}
& L_{3, \varepsilon, T}^{0}=-\frac{T^{2}}{2}\left\{{ }^{\prime} \nabla_{T / \varepsilon, e_{i}}^{\Lambda\left(T^{*(0,1)} Z\right) \otimes \xi}\right. \\
&+\frac{T}{\sqrt{2} \varepsilon^{2}}\left\langle S_{3, T / \varepsilon}\left(e_{i}\right) e_{j}, g_{\alpha}\right\rangle_{T / \varepsilon} c\left(e_{i}\right) g^{\alpha} \\
&+\frac{1}{\sqrt{2} \varepsilon}\left\langle S_{3, T / \varepsilon}\left(e_{i}\right) f_{\ell}, g_{\alpha}\right\rangle_{T / \varepsilon} c_{T / \varepsilon}\left(f_{\ell}\right) g^{\alpha} \\
&\left.+\frac{1}{2 \varepsilon^{2}}\left\langle S_{3, T / \varepsilon}\left(e_{i}\right) g_{\alpha}, g_{\beta}\right\rangle_{T / \varepsilon} g^{\alpha} \wedge g^{\beta}\right\}^{2} \\
&-\frac{\varepsilon^{2}}{2}\left\{{ }^{\prime} \nabla_{T / \varepsilon, f_{\ell}}^{\Lambda\left(T^{*(0,1)}\right.} Z\right) \otimes \xi \\
&+ \frac{1}{\sqrt{2} \varepsilon}\left\langle S_{3, T / \varepsilon}\left(f_{\ell}\right) f_{m}, g_{\alpha}\right\rangle_{T / \varepsilon} c_{T / \varepsilon}\left(f_{\ell}\right) g^{\alpha} \\
&+ \frac{T}{\sqrt{2} \varepsilon^{2}}\left\langle S_{3, T / \varepsilon}\left(f_{\ell}\right) e_{i}, g_{\alpha}\right\rangle_{T / \varepsilon} c\left(e_{i}\right) g^{\alpha} \\
&\left.+\frac{1}{2 \varepsilon^{2}}\left\langle S_{3, T / \varepsilon}\left(f_{\ell}\right) g_{\alpha}, g_{\beta}\right\rangle_{T / \varepsilon} g^{\alpha} \wedge g^{\beta}\right\}^{2}
\end{aligned}
$$

BULLETIN DE LA SOCIÉTÉ MATHÉMATIQUE DE FRANCE 


$$
\begin{aligned}
& +\frac{T^{2}}{8} K_{T / \varepsilon}^{Z}+\frac{1}{2} g^{\alpha} \wedge g^{\beta} \wedge L_{3, T / \varepsilon}^{\prime \xi}\left(g_{\alpha}, g_{\beta}\right) \\
& +\frac{\varepsilon^{2}}{4} c_{T / \varepsilon}\left(f_{\ell}\right) c_{T / \varepsilon}\left(f_{m}\right) L_{3, T / \varepsilon}^{\prime \xi}\left(f_{\ell}, f_{m}\right) \\
& +\frac{T^{2}}{4} c\left(e_{i}\right) c\left(e_{j}\right) L_{3, T / \varepsilon}^{\prime \xi}\left(e_{i}, e_{j}\right) \\
& +\frac{\varepsilon T}{2} c\left(e_{i}\right) c_{T / \varepsilon}\left(f_{\ell}\right) L_{3, T / \varepsilon}^{\prime \xi}\left(e_{i}, f_{\ell}\right) \\
& +\frac{T}{\sqrt{2}} g^{\alpha} \wedge c\left(e_{i}\right) L_{3, T / \varepsilon}^{\prime \xi}\left(g_{\alpha}, e_{i}\right) \\
& +\frac{\varepsilon}{\sqrt{2}} g^{\alpha} \wedge c_{T / \varepsilon}\left(f_{\ell}\right) L_{3, T / \varepsilon}^{\prime \xi}\left(g_{\alpha}, f_{\ell}\right)
\end{aligned}
$$

On rappelle que, pour $s \in S$, on note $\mathrm{d} v_{Z}, \mathrm{~d} v_{X}, \mathrm{~d} v_{Y}$ les formes de volume sur $Z, X, Y$ associées aux métriques $\pi_{1}^{*} g^{T Y} \oplus g^{T X}, g^{T X}, g^{T Y}$ sur $T Z=\pi_{1}^{*} T Y \oplus T X$, $T X, T Y$.

Soit $P_{3, \varepsilon, T}\left(x, x^{\prime}\right)\left(x, x^{\prime} \in Z_{s}\right)$ le noyau $\mathcal{C}^{\infty}$ de l'opérateur $\exp \left(-L_{3, \varepsilon, T}^{0}\right)$ relativement à $\frac{\mathrm{d} v_{Z}\left(x^{\prime}\right)}{(2 \pi)^{\operatorname{dim} Z}}$. Pour $a \in Y_{s}$, on pose

$$
g_{\varepsilon, T}(a)=\int_{X_{a}} \varphi \operatorname{Tr}_{s}\left[M_{3, \varepsilon, T}^{0} P_{3, \varepsilon, T}(x, x)\right] \frac{\mathrm{d} v_{X}(x)}{(2 \pi)^{\operatorname{dim} X}} .
$$

Par (7.3), on a :

$$
\varphi \operatorname{Tr}_{s}\left[\frac{1}{\varepsilon} M_{3, \varepsilon^{2}, T / \varepsilon} \exp \left(-B_{3, \varepsilon^{2}, T / \varepsilon}^{2}\right)\right]=\int_{Y_{s}} g_{\varepsilon, T} \frac{\mathrm{d} v_{Y}}{(2 \pi)^{\operatorname{dim} Y}} .
$$

Pour démontrer le théorème 4.8 , on va calculer la limite de $g_{\varepsilon, T}$ quand $\varepsilon$ tend vers zéro.

\section{b) Le changement d'échelle sur l'algèbre de Clifford.}

Grâce à la propriété de vitesse finie de propagation, on sait que le calcul de la limite de $g_{\varepsilon, T}$, pour $\varepsilon \rightarrow 0$, est local sur $Y_{s_{0}}\left(s_{0} \in S\right)$. Par conséquent, pour $b_{0} \in Y_{s_{0}}$, on peut remplacer $Y_{s_{0}} \operatorname{par}(T Y)_{b_{0}}=\mathbb{C}^{m_{0}}\left(m_{0}=\operatorname{dim} Y_{s}\right)$, avec $0 \in(T Y)_{b_{0}}$ representé par $b_{0}$.

On rappelle que $S_{1}, S_{2}, S_{3, T}$ sont les tenseurs définis au $\S 1$ a) associés à $\left(\pi_{1}, \widetilde{\omega}^{W}, W, V\right),\left(\pi_{2}, \omega^{V}, V, S\right),\left(\pi_{3}, \omega_{T}^{W}, W, S\right)$, et que l'application $\psi_{u}$ : $\Lambda\left(T_{\mathbb{R}}^{*} S\right) \rightarrow \Lambda\left(T_{\mathbb{R}}^{*} S\right)$ est définie en (5.44).

Soit $\nabla^{\pi_{2}^{*} \Lambda\left(T_{\mathbb{R}}^{*} S\right) \widehat{\otimes} \Lambda\left(T^{*(0,1)} Y\right)}$ la connexion sur $\pi_{2}^{*} \Lambda\left(T_{\mathbb{R}}^{*} S\right) \widehat{\otimes} \Lambda\left(T^{*(0,1)} Y\right)$ le long de la fibre $Y$ qui est induite par $\nabla^{\Lambda\left(T^{*(0,1)} Y\right)}$.

TOME $127-1999-\mathrm{N}^{\circ} 4$ 
DÉfinition 7.1. - Soit ' $\nabla^{\pi_{2}^{*} \Lambda\left(T_{\mathbb{R}}^{*} S\right) \widehat{\otimes} \Lambda\left(T^{*(0,1)} Y\right)}$ la connexion le long de la fibre $Y$ sur $\pi_{2}^{*} \Lambda\left(T_{\mathbb{R}}^{*} S\right) \widehat{\otimes} \Lambda\left(T^{*(0,1)} Y\right)$

$$
\begin{aligned}
& { }^{\prime} \nabla^{\pi_{2}^{*} \Lambda\left(T_{\mathbb{R}}^{*} S\right) \widehat{\otimes} \Lambda\left(T^{*(0,1)} Y\right)}=\nabla^{\pi_{2}^{*} \Lambda\left(T_{\mathbb{R}}^{*} S\right) \widehat{\otimes} \Lambda\left(T^{*(0,1)} Y\right)} \\
& +\frac{1}{2}\left\langle S_{2}(\cdot) f_{m}, g_{\alpha, 2}^{H}\right\rangle \sqrt{2} c\left(f_{m}\right) g^{\alpha} \\
& +\frac{1}{2}\left\langle S_{2}(\cdot) g_{\alpha, 2}^{H}, g_{\beta, 2}^{H}\right\rangle g^{\alpha} \wedge g^{\beta} \text {. }
\end{aligned}
$$

Soit $\nabla^{\oplus}$ la connexion sur

$$
\begin{aligned}
\pi_{3}^{*} \Lambda\left(T_{\mathbb{R}}^{*} S\right) \widehat{\otimes} \Lambda\left(T^{*(0,1)} Z\right) & \otimes \xi \\
& \simeq \pi_{1}^{*}\left(\pi_{2}^{*} \Lambda\left(T_{\mathbb{R}}^{*} S\right) \widehat{\otimes} \Lambda\left(T^{*(0,1)} Y\right)\right) \widehat{\otimes}\left(\Lambda\left(T^{*(0,1)} X\right) \otimes \xi\right)
\end{aligned}
$$

le long de la fibre $Z$

$$
\nabla^{\oplus}=\pi_{1}^{* \prime} \nabla^{\pi_{2}^{*} \Lambda\left(T_{\mathbb{R}}^{*} S\right) \widehat{\otimes} \Lambda\left(T^{*(0,1)} Y\right)} \otimes 1+1 \otimes \nabla^{\Lambda\left(T^{*(0,1)} X\right) \otimes \xi} .
$$

Pour $y \in \mathbb{C}^{m_{0}}$, soit $Y=y+\bar{y}$. On relève la trajectoire $t \in \mathbb{R}_{+}^{*} \mapsto t Y$ horizontalement en la trajectoire $t \in \mathbb{R}_{+}^{*} \mapsto x_{t} \in Z_{s}$ avec $x_{t} \in X_{t Y}$ et $\mathrm{d} x / \mathrm{d} t \in$ $T^{H} Z$. Pour $x_{0} \in X_{0}$, on identifie $T X_{x_{t}}$ et $\left(\pi_{3}^{*} \Lambda\left(T_{\mathbb{R}}^{*} S\right) \widehat{\otimes} \Lambda\left(T^{*(0,1)} Z\right) \otimes \xi\right)_{x_{t}}$ à

$$
T X_{x_{0}} \quad \text { et } \quad\left(\pi_{2}^{*} \Lambda\left(T_{\mathbb{R}}^{*} S\right) \widehat{\otimes} \Lambda\left(T^{*(0,1)} Y\right)\right)_{b_{0}} \widehat{\otimes}\left(\Lambda\left(T^{*(0,1)} X\right) \otimes \xi\right)_{x_{0}}
$$

par transport parallèle le long de la courbe $t \mapsto x_{t} \in Z_{s}$ relativement à $\nabla^{T X}$ et $\psi_{\varepsilon} \nabla^{\oplus} \psi_{\varepsilon}^{-1}$.

L'opérateur $L_{3, \varepsilon, T}^{0}$ agit sur l'espace vectoriel $H_{b_{0}}$ des sections $\mathcal{C}^{\infty} \operatorname{de}\left(\pi_{2}^{*} \Lambda\left(T_{\mathbb{R}}^{*} S\right)\right.$ $\left.\widehat{\otimes} \Lambda\left(T^{*(0,1)} Y\right)\right)_{b_{0}} \widehat{\otimes}\left(\Lambda\left(T^{*(0,1)} X\right) \otimes \xi\right)_{X_{b_{0}}}$ sur $\mathbb{R}^{2 m_{0}} \times X_{b_{0}}$.

Pour $s \in H_{b_{0}}$, on pose

$$
\left\{\begin{array}{l}
\left(F_{\varepsilon} s\right)(Y, x)=s\left(\frac{Y}{\varepsilon}, x\right) \quad \text { pour } \quad(Y, x) \in \mathbb{R}^{2 m_{0}} \times X_{b_{0}}, \\
L_{3, \varepsilon, T}^{2}=F_{\varepsilon}^{-1} L_{3, \varepsilon, T}^{0} F_{\varepsilon} .
\end{array}\right.
$$

Soit $\mathcal{O}_{p}$ l'ensemble des opérateurs différentiels agissant sur les sections $\mathcal{C}^{\infty}$ de $\left.\left(\Lambda\left(T^{*(0,1)} X\right) \otimes \xi\right)\right)_{\mid X_{b_{0}}}$ sur $\mathbb{R}^{2 m_{0}} \times X_{b_{0}}$. Soit $c\left(T_{\mathbb{R}} Y\right)$ l'algèbre de Clifford de $\left(T_{\mathbb{R}} Y, g^{T_{\mathbb{R}} Y}\right)$. Alors on a

$$
L_{3, \varepsilon, T}^{2} \in \pi_{2}^{*} \Lambda\left(T_{\mathbb{R}}^{*} S\right) \widehat{\otimes} c\left(T_{\mathbb{R}} Y\right)_{b_{0}} \widehat{\otimes} \mathcal{O}_{p}
$$

Définition 7.2. - Pour $\varepsilon>0$, on pose

$$
\tilde{c}_{\varepsilon}\left(f_{\ell}\right)=\frac{\sqrt{2}}{\varepsilon} f^{\ell} \wedge-\frac{\varepsilon}{\sqrt{2}} i_{f_{\ell}} .
$$

Soient $L_{3, \varepsilon, T}^{3}$ et $M_{3, \varepsilon, T}^{3} \in\left(\pi_{2}^{*}\left(\Lambda T_{\mathbb{R}}^{*} S\right) \widehat{\otimes} c\left(T_{\mathbb{R}} Y\right)\right)_{b_{0}} \widehat{\otimes} \mathcal{O}_{p}$ les opérateurs obtenus en remplaçant $c\left(f_{\ell}\right)$ par $\tilde{c}_{\varepsilon}\left(f_{\ell}\right)$ dans $L_{3, \varepsilon, T}^{2}$ et $M_{3, \varepsilon, T}^{0}$. 
Soit $\mathrm{d} v_{T Y \mid b_{0}}$ la forme de volume de $\left(T_{\mathbb{R}} Y_{b_{0}}, g^{T Y_{b_{0}}}\right) \simeq \mathbb{R}^{2 m_{0}}$.

Soit $P_{3, \varepsilon, T}^{3}\left((Y, x),\left(Y^{\prime}, x^{\prime}\right)\right)\left((Y, x),\left(Y^{\prime}, x^{\prime}\right) \in \mathbb{R}^{2 m_{0}} \times X_{b_{0}}\right)$ le noyau $\mathcal{C}^{\infty}$ de l'opérateur $\exp \left(-L_{3, \varepsilon, T}^{3}\right)$ relativement à $\mathrm{d} v\left(Y^{\prime}\right)_{\left.T Y\right|_{b_{0}}} \mathrm{~d} v\left(x^{\prime}\right)_{T X}{\mid X_{b_{0}}} /(2 \pi)^{\operatorname{dim} Z}$. Alors pour $x \in X_{b_{0}}$, il existe

$$
R_{\varepsilon, T}^{i_{1} \cdots i_{p}, j_{1} \cdots j_{q}}((0, x),(0, x)) \in \Lambda\left(T_{\mathbb{R}}^{*} S\right) \widehat{\otimes} \operatorname{End}\left(\Lambda\left(T^{*(0,1)} X\right) \otimes \xi\right)_{\mid X_{b_{0}}}
$$

tels que $M_{3, \varepsilon, T}^{3} P_{3, \varepsilon, T}^{3}((0, x),(0, x))$ peut s'écrire sous la formule suivante :

$$
\begin{aligned}
& M_{3, \varepsilon, T}^{3} P_{3, \varepsilon, T}^{3}((0, x),(0, x)) \\
& \quad=\sum_{\substack{1 \leq i_{1}<\cdots<i_{p} \leq 2 m_{0} \\
1 \leq j_{1}<\cdots<j_{q} \leq 2 m_{0}}} f^{i_{1}} \wedge \ldots \wedge f^{i_{p}} \wedge i_{f_{j_{1}}} \ldots i_{f_{j_{q}}} \widehat{\otimes} R_{\varepsilon, T}^{i_{1}, \ldots, i_{p}, j_{1}, \ldots, j_{q}} .
\end{aligned}
$$

On pose

$$
\left[M_{3, \varepsilon, T}^{3} P_{3, \varepsilon, T}^{3}((0, x),(0, x))\right]^{\max }=R_{\varepsilon, T}^{1, \ldots, 2 m_{0}}((0, x),(0, x)) .
$$

D'après [ABoP, p. 484] et [Ge], on sait que

$$
\begin{aligned}
\varphi \operatorname{Tr}_{s}\left[M_{3, \varepsilon, T}^{0} P_{3, \varepsilon, T}((0, x),(0, x))\right] \\
=(-i)^{\operatorname{dim} Y} \varphi \operatorname{Tr}_{s}\left[\left[M_{3, \varepsilon, T}^{3} P_{3, \varepsilon, T}^{3}((0, x),(0, x))\right]^{\max }\right] .
\end{aligned}
$$

\section{zéro.}

c) L'asymptotique des opérateurs $L_{3, \epsilon, T}^{3}$ et $M_{3, \epsilon, T}^{3}$ quand $\epsilon$ tend vers

En utilisant (1.8), (7.4) et (7.15), on sait que, quand $\varepsilon \rightarrow 0$

$$
\begin{aligned}
M_{3, \varepsilon, T}^{3} \longrightarrow & M_{3,0, T}^{3} \\
= & \frac{2}{T}\left(N_{X}-\operatorname{dim} X\right)+\frac{2 i}{T^{3}} \widetilde{\omega}^{W}\left(g_{\alpha, 3, \infty}^{H}, f_{\ell, 1}^{H}\right) g^{\alpha} \wedge f^{\ell} \\
& +\frac{i}{T^{3}} \widetilde{\omega}^{W}\left(g_{\alpha, 3, \infty}^{H}, g_{\beta, 3, \infty}^{H}\right) g^{\alpha} \wedge g^{\beta}-\frac{2}{T^{3}}\left\langle h \bar{\theta}_{\ell}, \theta_{m}\right\rangle_{g^{T Y}} \bar{\theta}^{\ell} \wedge \theta^{m} .
\end{aligned}
$$

Par [BerB, (7.27)] et (7.19), on a

$$
M_{3,0, T}^{3}=\frac{2}{T}\left(N_{X}-\operatorname{dim} X\right)+\frac{2 i}{T^{3}} \widetilde{\omega}_{1}^{H \bar{H}}=\frac{2}{T}\left(\widetilde{N}_{1, T^{2}}-\operatorname{dim} X\right) .
$$

Soit $\Gamma_{2}^{\pi_{2}^{*} \Lambda\left(T_{\mathbb{R}}^{*} S\right) \widehat{\otimes} \Lambda\left(T^{*(0,1)} Y\right)}$ la forme de connexion sur $\mathbb{C}^{m_{0}}$ de

$$
\pi_{2}^{*} \Lambda\left(T_{\mathbb{R}}^{*} S\right) \widehat{\otimes} \Lambda\left(T^{*(0,1)} Y\right)
$$

TOME $127-1999-\mathrm{N}^{\circ} 4$ 
relativement à ${ }^{\prime} \nabla^{\pi_{2}^{*} \Lambda\left(T_{\mathbb{R}}^{*} S\right) \widehat{\otimes} \Lambda\left(T^{*(0,1)} Y\right)}$ le long de la courbe $t \mapsto t Y\left(Y \in \mathbb{R}^{2 m_{0}}\right)$. Alors par [ABoP, prop. 3.7], pour $Y \in \mathbb{R}^{2 m_{0}}$, on a

$$
\begin{aligned}
\Gamma_{Y}^{\pi_{2}^{*} \Lambda\left(T_{\mathbb{R}}^{*} S\right) \widehat{\otimes} \Lambda\left(T^{*(0,1)} Y\right)}(\cdot) & \\
= & \frac{1}{2}{ }^{\prime} \nabla^{\pi_{2}^{*} \Lambda\left(T_{\mathbb{R}}^{*} S\right) \widehat{\otimes} \Lambda\left(T^{*(0,1)} Y\right), 2}(Y, \cdot)+O\left(|Y|^{2}\right) .
\end{aligned}
$$

Soit $P^{T Y}$ la projection de $T V=T_{2}^{H} V \oplus T Y$ sur $T Y$.

Pour $X \in T_{\mathbb{R}} Z$, on note $X^{(1,0)}, X^{(0,1)}$ les composantes de $X$ dans $T Z, \overline{T Z}$. Soit

$$
L_{1}^{\prime \xi}=L^{\xi}+\frac{1}{2} \operatorname{Tr}\left[R^{T X}\right]
$$

Soit $K^{X}$ la courbure scalaire de la fibre $\left(X, g^{T X}\right)$. Pour $U \in T_{\mathbb{R}} Y$, soit $\nabla_{U}$ l'opérateur différentiel ordinaire dans la direction $U$.

En utilisant [B4, th. 11.8 et (11.61)], le théorème 1.7, (1.35), (7.6), (7.21) et (7.22), quand $\varepsilon \rightarrow 0$, on a :

$$
\begin{aligned}
& L_{3, \varepsilon, T}^{3} \longrightarrow L_{3,0, T}^{3} \\
& =-\frac{1}{2}\left(\nabla_{f_{\ell}}+\frac{1}{2}\left\langle R^{T Y} Y, f_{\ell}\right\rangle_{g_{b_{0}}^{T Y}}\right)^{2}+\frac{1}{2} \operatorname{Tr}\left[\nabla^{T Y, 2}\right] \\
& -\frac{T^{2}}{2}\left\{\nabla_{e_{i}}^{\Lambda\left(T^{*(0,1)} X\right) \otimes \xi}+\frac{1}{T \sqrt{2}}\left\langle A\left(e_{i}\right) f_{\ell, 1}^{H}, e_{j}\right\rangle f^{\ell} \wedge c\left(e_{j}\right)\right. \\
& -\frac{i}{T^{2}} \sum_{\ell<m} e_{i}^{(1,0)} \widetilde{\omega}_{1}^{H \bar{H}}\left(f_{\ell}, f_{m}\right) f^{\ell} \wedge f^{m} \\
& +\frac{1}{T \sqrt{2}}\left\langle S_{1}\left(e_{i}\right) e_{j}, g_{\alpha, 3, \infty}^{H}\right\rangle c\left(e_{j}\right) g^{\alpha} \\
& +\frac{1}{2 T^{2}}\left\langle S_{1}\left(e_{i}\right) g_{\alpha, 3, \infty}^{H}, g_{\beta, 3, \infty}^{H}\right\rangle g^{\alpha} \wedge g^{\beta} \\
& +\frac{1}{T^{2}}\left[\left\langle S_{1}\left(e_{i}\right) f_{\ell, 1}^{H}, g_{\alpha, 3, \infty}^{H}\right\rangle\right. \\
& \left.\left.+\frac{1}{2} L_{e_{i}}\left\langle h^{\prime} g_{\alpha, 3, \infty}^{H}, f_{\ell, 1}^{H}\right\rangle_{\pi_{1}^{*} g^{T Y}}\right] f^{\ell} \wedge g^{\alpha}\right\}^{2} \\
& +\frac{T^{2}}{8} K^{X}+\frac{1}{2} g^{\alpha} \wedge g^{\beta} \wedge L_{1}^{\prime \xi}\left(g_{\alpha, 3, \infty}^{H}, g_{\beta, 3, \infty}^{H}\right) \\
& +\frac{1}{2} f^{\ell} \wedge f^{m} \wedge L_{1}^{\prime \xi}\left(f_{\ell, 1}^{H}, f_{m, 1}^{H}\right) \\
& +\frac{T^{2}}{4} c\left(e_{i}\right) c\left(e_{j}\right) L_{1}^{\prime \xi}\left(e_{i}, e_{j}\right)+\frac{T}{\sqrt{2}} f^{\ell} \wedge c\left(e_{i}\right) L_{1}^{\prime \xi}\left(f_{\ell, 1}^{H}, e_{i}\right) \\
& +\frac{T}{\sqrt{2}} g^{\alpha} \wedge c\left(e_{i}\right) L_{1}^{\prime \xi}\left(g_{\alpha, 3, \infty}^{H}, e_{i}\right)+g^{\alpha} \wedge f^{\ell} \wedge L_{1}^{\prime \xi}\left(g_{\alpha, 3, \infty}^{H}, f_{\ell, 1}^{H}\right) .
\end{aligned}
$$




\section{d) La superconnexion de Levi-Civita.}

On rappelle que $B_{1, u}$ est la superconnexion de Levi-Civita associée à $\left(\pi_{1}, \widetilde{\omega}^{W}, h^{\xi}\right)$, qui a été définie à la définition 2.3. On pose :

$$
\widetilde{\omega}^{H \bar{H}}=\frac{1}{2} i \widetilde{\omega}_{1}^{H \bar{H}}\left(f_{\ell}, f_{m}\right) f^{\ell} \wedge f^{m}-\frac{1}{2}\left\langle h^{\prime} g_{\alpha, 3, \infty}^{H}, f_{\ell, 1}^{H}\right\rangle_{\pi_{1}^{*} g^{T Y}} f^{\ell} \wedge g^{\alpha} .
$$

En utilisant [BerB, (7.33)], (7.23) et en procédant comme dans [BerB, $\S 7(\mathrm{c})]$, on a :

$$
\begin{aligned}
\mathrm{e}^{-\frac{1}{T^{2}} \widetilde{\omega}^{H \bar{H}}} L_{3,0, T}^{3} & \mathrm{e}^{\frac{1}{T^{2}} \widetilde{\omega}^{H \bar{H}}} \\
& =-\frac{1}{2}\left(\nabla_{f_{\ell}}+\frac{1}{2}\left\langle R^{T Y} Y, f_{\ell}\right\rangle_{g_{b_{0}}^{T Y}}\right)^{2}+\frac{1}{2} \operatorname{Tr}\left[R^{T Y}\right]+B_{1, T^{2}}^{2}
\end{aligned}
$$

\section{e) Preuve du théorème 4.8.}

En utilisant (7.10), (7.18), (7.19), (7.23) et (7.25), la preuve du théorème 4.8 est la même que dans [BerB, $\S 7$ (d)].

\section{Preuves des théorèmes 4.9 et 4.10}

Le but de ce paragraphe est d'établir les théorèmes 4.9 et 4.10 .

Ce paragraphe est organisé de la façon suivante. Dans a), on énonce le théorème 8.1 , dont on peut déduire le théorème 4.10. Les parties b), c) sont consacrées à la preuve du théorème 8.1. Dans b), en utilisant la propriété de vitesse finie de propagation, on montre que la preuve du théorème 8.1 est locale sur la fibre $Y_{s}$. Dans c), en utilisant le théorème d'indice local relatif, on montre le théorème 8.1 et la première partie du théorème 4.9. Dans $\mathrm{d}$ ), on montre la deuxième partie du théorème 4.9 .

On fait les mêmes hypothèses et on utilise les mêmes notations qu'aux paragraphes $1 \mathrm{~b}), 5$ et 7 .

\section{a) Reformulation du théorème 4.10.}

On rappelle que $\mu_{0}(T)$ est la forme sur $S$ définie au théorème 4.9 .

ThÉORÈme 8.1. - Il existe $C>0$ tel que, pour tout $u \in] 0,1]$ et $T \geq 1$, on a

$$
\begin{aligned}
& \text { (8.1) } \mid \varphi \operatorname{Tr}_{s}\left[M_{3, u^{2} / T^{2}, T} \exp \left(-B_{3, u^{2} / T^{2}, T}^{2}\right)\right] \\
& -\frac{2}{u^{2}} \int_{Z} \frac{\widetilde{\omega}^{W}}{2 \pi T} \operatorname{Td}\left(T Z, g_{T}^{T Z}\right) \operatorname{ch}\left(\xi, h^{\xi}\right) \\
& +\int_{Z} \frac{\partial}{\partial b} \operatorname{Td}\left(\frac{-R_{T}^{T Z}}{2 i \pi}-b\left(g_{T}^{T Z}\right)^{-1} \frac{\partial}{\partial T}\left(g_{T}^{T Z}\right)\right)_{b=0} \operatorname{ch}\left(\xi, h^{\xi}\right)+\varphi \mathrm{d}^{S} \mu_{0}(T) \mid \leq \frac{C u^{2}}{T} . \\
& \quad \text { томе } 127-1999-\mathrm{N}^{\circ} 4
\end{aligned}
$$


Remarque 8.2. - Le théorème 8.1 implique le théorème 4.10. En effet, pour $0<\varepsilon \leq 1$ et $\varepsilon \leq T \leq 1$, on applique (8.1) pour $u=T$, et $T$ étant remplacé par $T / \varepsilon$; alors le terme à droite de (8.1) est dominé par $C T^{2} \varepsilon / T=C \varepsilon T \leq C \varepsilon$. On a donc démontré le théorème 4.10.

\section{b) La preuve du théorème 8.1 est locale sur $Y_{s}$.}

Soit $r$ tel que

$$
r<\inf _{s \in S}\left\{\text { rayon d'injectivité des fibres }\left(Y_{s}, g^{T Y}\right)\right\} .
$$

Soit $\left.\alpha \in] 0, \frac{1}{4} r\right]$. Pour $b \in V$, soit $B^{Y}(b, \alpha)$ la boule dans $Y_{s}$ de centre $b$ et de rayon $\alpha$. Soit $f: \mathbb{R} \rightarrow[0,1]$ une fonction paire $\mathcal{C}^{\infty}$, telle que

$$
f(t)= \begin{cases}1 & \text { si }|t| \leq \frac{1}{2} \alpha \\ 0 & \text { si }|t| \geq \alpha\end{cases}
$$

On pose :

$$
g=1-f
$$

DÉfinition 8.3. - Pour $u \in] 0,1]$ et $a \in \mathbb{C}$, on pose :

$$
\left\{\begin{array}{l}
F_{u}(a)=\int_{-\infty}^{+\infty} \exp (i t a \sqrt{2}) \exp \left(-\frac{1}{2} t^{2}\right) f(u t) \frac{\mathrm{d} t}{\sqrt{2 \pi}}, \\
G_{u}(a)=\int_{-\infty}^{+\infty} \exp (i t a \sqrt{2}) \exp \left(-\frac{1}{2} t^{2}\right) g(u t) \frac{\mathrm{d} t}{\sqrt{2 \pi}} .
\end{array}\right.
$$

On a

$$
F_{u}(a)+G_{u}(a)=\exp \left(-a^{2}\right) .
$$

Les fonctions $F_{u}(a), G_{u}(a)$ sont des fonctions holomorphes paires sur $\mathbb{C}$. Il existe donc des fonctions holomorphes $\widetilde{F}_{u}(a), \widetilde{G}_{u}(a)$ telles que

$$
F_{u}(a)=\widetilde{F}_{u}\left(a^{2}\right), \quad G_{u}(a)=\widetilde{G}_{u}\left(a^{2}\right) .
$$

Par (8.5), (8.6), on a :

$$
\exp \left(-B_{3, u^{2} / T^{2}, T}^{2}\right)=\widetilde{F}_{u / T}\left(B_{3, u^{2} / T^{2}, T}^{2}\right)+\widetilde{G}_{u / T}\left(B_{3, u^{2} / T^{2}, T}^{2}\right) .
$$

Proposition 8.4. - Il existe c, $C>0$ tels que, pour tout $0<u \leq 1$ et tout $T \geq 1$

$$
\left|\operatorname{Tr}_{s}\left[M_{3, u^{2} / T^{2}, T} \widetilde{G}_{u / T}\left(B_{3, u^{2} / T^{2}, T}^{2}\right)\right]\right| \leq C \exp \left(-c \frac{T^{2}}{u^{2}}\right)
$$


Preuve. - Pour $v>0$, on pose

$$
H_{v}(a)=\int_{-\infty}^{+\infty} \exp (i t \sqrt{2} a) \exp \left(-\frac{t^{2}}{2 v^{2}}\right) g(t) \frac{\mathrm{d} t}{v \sqrt{2 \pi}}
$$

Alors il existe une fonction holomorphe $\widetilde{H}_{v}(a)$ telle que $H_{v}(a)=\widetilde{H}_{v}\left(a^{2}\right)$. Par (8.6) et $(8.9)$, on a

$$
\widetilde{G}_{v}(a)=\widetilde{H}_{v}\left(\frac{a}{v^{2}}\right)
$$

D'après $(5.2),(5.45)$ et $(8.10)$, on a

(8.11) $\operatorname{Tr}_{s}\left[M_{3, u^{2} / T^{2}, T} \widetilde{G}_{u / T}\left(-B_{3, u^{2} / T^{2}, T}^{2}\right)\right]=\psi_{u / T} \operatorname{Tr}_{s}\left[C_{T} M_{3,1, T} C_{T}^{-1} \widetilde{H}_{u / T}\left(A_{T}^{2}\right)\right]$.

Soit $\Delta$ le contour suivant dans $\mathbb{C}$ :

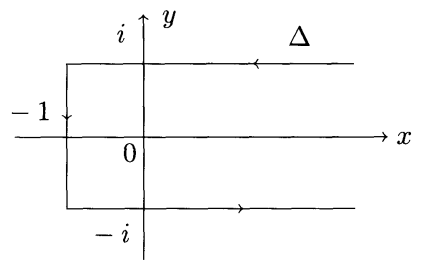

Par [B4, (11.21)], pour tout $m \in \mathbb{N}$, il existe $c_{m}>0$ et $C_{m}>0$ tels que

$$
\sup _{a \in \Delta}|a|^{m} \cdot\left|\widetilde{H}_{v}(a)\right| \leq C_{m} \exp \left(-\frac{c_{m}}{v^{2}}\right)
$$

On a aussi

$$
\widetilde{H}_{u / T}\left(A_{T}^{2}\right)=\frac{1}{2 \pi i} \int_{\Delta} \frac{\widetilde{H}_{u / T}(\lambda)}{\lambda-A_{T}^{2}} \mathrm{~d} \lambda .
$$

En utilisant (5.69), (8.12), (8.13), et en procédant comme dans le $\S 5 \mathrm{~g}$ ), on voit facilement que, pour $u \in] 0,1]$ et $T \geq 1$,

$$
\left|\operatorname{Tr}_{s}\left[C_{T} M_{3,1, T} C_{T}^{-1} \widetilde{H}_{u / T}\left(A_{T}^{2}\right)\right]\right| \leq C \exp \left(-c \frac{T^{2}}{u^{2}}\right)
$$

De (8.11) et (8.14), on tire la proposition.

$$
\text { TOME } 127-1999-\mathrm{N}^{\circ} 4
$$


Soit $\widetilde{F}_{u / T}\left(B_{3, u^{2} / T^{2}, T}^{2}\right)\left(x, x^{\prime}\right)\left(x, x^{\prime} \in Z_{s}\right)$ le noyau de $\widetilde{F}_{u / T}\left(B_{3, u^{2} / T^{2}, T}^{2}\right)$ relativement à $\mathrm{d} v_{Z}\left(x^{\prime}\right) /(2 \pi)^{\operatorname{dim} Z}$. En utilisant (8.4) et la propriété de vitesse finie de propagation, [CP, $\S 7.8]$ et $[\mathrm{T}, \S 4.4]$, il est clair que pour $0<u \leq 1, T \geq 1$ et $x \in Z_{s}, \widetilde{F}_{u / T}\left(B_{3, u^{2} / T^{2}, T}^{2}\right)(x, x)$ ne dépend que de la restriction de $B_{3, u^{2} / T^{2}, T}^{2}$ à $\pi_{1}^{-1} B^{Y}\left(\pi_{1} x, \alpha\right)$. Par (8.8) et par la discussion ci-dessus, la démonstration de (8.1) est locale sur $Y_{s}$.

Pour $b_{0} \in Y_{s_{0}}$, on peut remplacer $Z_{s_{0}}$ par $\mathbb{C}^{m_{0}} \times X_{b_{0}}$ comme au $\S 7 \mathrm{~b}$ ), et on trivialise les fibrés vectoriels comme indiqué au $\S 7 \mathrm{~b}$ ). On va montrer le théorème 8.1 dans cette situation.

c) Preuve du théorème 8.1.

On rappelle que $E_{1,0}$ est la forme $\mathcal{C}^{\infty}$ sur $V$ définie en (4.20).

THÉORÈME 8.5.

i) Il existe $C>0$, et des formes $a_{T, j}($ avec $n=\operatorname{dim} Z$ et $j \geq-n), \mathcal{C}^{\infty}$ sur $S$, qui dépendent continûment de $T \in[1,+\infty]$, tels que, pour tout $u \in] 0,1]$ et $T \in[1,+\infty[$,

$$
\left|\varphi \operatorname{Tr}_{s}\left[M_{3, u^{2} / T^{2}, T} \exp \left(-B_{3, u^{2} / T^{2}, T}^{2}\right)\right]-\sum_{j=-n}^{0} \frac{a_{T, j}}{T} u^{2 j}\right| \leq C \frac{u^{2}}{T} .
$$

ii) Pour $j \geq-n$, quand $T \rightarrow+\infty$, on a

$$
a_{T, j}=a_{\infty, j}+O\left(\frac{1}{T}\right)
$$

iii) De plus

$$
a_{\infty, 0}=\int_{Y} \operatorname{Td}\left(T Y, g^{T Y}\right)
$$

Preuve. - i) En utilisant (5.45) et (7.2), on a

$$
\begin{aligned}
\varphi \operatorname{Tr}_{s}\left[M_{3, u^{2} / T^{2}, T} \exp \left(-B_{3, u^{2} / T^{2}, T}^{2}\right)\right] \\
\quad=\psi_{u} \varphi \operatorname{Tr}_{s}\left[\frac{1}{T} M_{3, \frac{1}{T}, 1}^{0} \exp \left(-u^{2} L_{3, \frac{1}{T}, 1}^{0}\right)\right] .
\end{aligned}
$$

On va utiliser les mêmes notations du $\S 7$, avec $\varepsilon$ remplacé par $1 / T$, et $T$ par 1 . Alors d'après (7.23), quand $T \rightarrow+\infty$,

$$
L_{3, \frac{1}{T}, 1}^{3} \longrightarrow L_{3,0,1}^{3} \text {. }
$$

Soit $P_{\varepsilon, T, u}$ (resp. $P_{\varepsilon, T, u}^{3}$ ) le noyau $\mathcal{C}^{\infty}$ de l'opérateur $\exp \left(-u^{2} L_{3, \varepsilon, T}^{0}\right)$ (resp. $\left.\exp \left(-u^{2} L_{3, \varepsilon, T}^{3}\right)\right)$ relativement à $\mathrm{d} v_{T Y \mid b_{0}} \mathrm{~d} v_{X_{b_{0}}} /(2 \pi)^{\operatorname{dim} Z}$. 
D'après (7.19), quand $T \rightarrow+\infty$,

$$
M_{3, \frac{1}{T}, 1}^{3} \longrightarrow M_{3,0,1}^{3}=2\left(\widetilde{N}_{1,1}-\operatorname{dim} X\right) .
$$

En utilisant (8.19), (8.20), et en procédant comme en [BerB, $\S 8(\mathrm{c})$ ], on sait qu'il existe $C>0$, et des sections $a_{T, j}^{\prime}$ de $\pi_{3}^{*} \Lambda\left(T_{\mathbb{R}}^{*} S\right) \widehat{\otimes} \pi_{1}^{*} \Lambda\left(T_{\mathbb{R}}^{*} Y\right)$ sur $Z_{s}$ qui dépendent continûment de $T \in[1,+\infty]$, tels que pour tout $u \in] 0,1], T \in[1,+\infty]$ et $x \in X_{b_{0}}$,

$$
\left|\psi_{u} \operatorname{Tr}_{s}\left[M_{3, \frac{1}{T}, 1}^{3} P_{\frac{1}{T}, 1, u}^{3}((0, x),(0, x))\right]-\sum_{j=-n}^{0} a_{T, j}^{\prime}(x) u^{2 j}\right| \leq c u^{2} .
$$

Posons

$$
a_{T, j}=(-i)^{\operatorname{dim} Y} \varphi \int_{Z}\left[a_{T, j}^{\prime}(x)\right]^{\max } \frac{\mathrm{d} v_{Z}(x)}{(2 \pi)^{\operatorname{dim} Z}} .
$$

Comme en (7.20), on a aussi

$$
\begin{aligned}
\varphi \operatorname{Tr}_{s}\left[\frac{1}{T} M_{3, \frac{1}{T}, 1}^{0} P_{\frac{1}{T}, 1, u}((0, x),(0, x))\right] \\
=(-i)^{\operatorname{dim} Y} \varphi \operatorname{Tr}_{s}\left[\left\{\frac{1}{T} M_{3, \frac{1}{T}, 1}^{3} P_{\frac{1}{T}, 1, u}^{3}((0, x),(0, x))\right\}^{\max }\right] .
\end{aligned}
$$

En utilisant (8.18), (8.21) et (8.23), on en déduit (8.15).

ii) Par l'asymptotique du noyau de chaleur [BGS2, th. 2.11], dans (8.21), $a_{T, j}^{\prime}(x)\left(x \in X_{b_{0}}\right)$ sont des fonctions des coefficients de $M_{3, \frac{1}{T}, 1}^{3}, L_{3, \frac{1}{T}, 1}^{3}$, et de ses dérivées au point $(0, x)$. Comme $L_{3, \varepsilon, 1}^{3}$ et $M_{3, \varepsilon, 1}^{3}$ sont $\mathcal{C}^{\infty}$ pour $\varepsilon \in[0,1]$, on a

$$
a_{T, j}^{\prime}(x)=a_{\infty, j}^{\prime}(x)+O\left(\frac{1}{T}\right) .
$$

De (8.22), (8.24), on tire (8.16).

iii) Soit $q_{u^{2}}\left(x, x^{\prime}\right)\left(x, x^{\prime} \in X\right)$ le noyau $\mathcal{C}^{\infty}$ de l'opérateur $\exp \left(-B_{1, u^{2}}^{2}\right)$ relativement à $\mathrm{d} v_{X}\left(x^{\prime}\right) /(2 \pi)^{\operatorname{dim} X}$. En utilisant (7.25), (8.20), et en procédant comme en [BerB, $\S 7(\mathrm{~d})]$, on a

$$
\begin{aligned}
\psi_{u} \operatorname{Tr}_{s}\left[M_{3,0,1}^{3} P_{0,1, u}^{3}(\right. & (0, x),(0, x))] \\
& =2 \operatorname{Td}\left(-R^{T Y}\right)_{b_{0}} \operatorname{Tr}_{s}\left[\left(\widetilde{N}_{1, u^{2}}-\operatorname{dim} X\right) q_{u^{2}}(x, x)\right] .
\end{aligned}
$$

D'après [BGS2, th. 2.16], (8.21) et (8.25), on a

$$
\begin{aligned}
\varphi_{1}\left(\int_{X} a_{\infty, 0}^{\prime} \frac{\mathrm{d} v_{X}}{(2 \pi)^{\operatorname{dim} X}}\right) \\
\quad=\operatorname{Td}\left(T Y, g^{T Y}\right)\left[\pi_{1 *}\left(-2 \operatorname{Td}^{\prime}\left(T X, g^{T X}\right) \operatorname{ch}\left(\xi, h^{\xi}\right)\right)-\varphi_{1} d^{V} E_{1,0}\right] .
\end{aligned}
$$

Par (8.22) et (8.26), on a (8.17).

On a bien terminé la preuve du théorème 8.5.

TOME $127-1999-\mathrm{N}^{\circ} 4$ 
Soient

$$
\left\{\begin{array}{l}
C_{3,-1}(T)=\int_{Z} \frac{\widetilde{\omega}^{W}}{2 \pi} \operatorname{Td}\left(T Z, g_{T}^{T Z}\right) \operatorname{ch}\left(\xi, h^{\xi}\right) \\
C_{3,0}(T)=\int_{Z} \frac{\partial}{\partial b} \operatorname{Td}\left(-\frac{R_{T}^{T Z}}{2 \pi i}-b\left(g_{T}^{T Z}\right)^{-1} \frac{\partial}{\partial T}\left(g_{T}^{T Z}\right)\right)_{\mid b=0} \operatorname{ch}\left(\xi, h^{\xi}\right)
\end{array}\right.
$$

Proposition 8.6.

i) Il existe une forme $\mu_{0}(T), \mathcal{C}^{\infty}$ sur $S$, telle que, pour $T$ fixé, quand $u \rightarrow 0$,

$$
\operatorname{Tr}_{s}\left[u M_{3, u, T} \exp \left(-B_{3, u, T}^{2}+d u \frac{\partial}{\partial u} B_{3, u, T}\right)\right]^{d u}=\mu_{0}(T)+O(u)
$$

ii) Pour $T \geq 1$ fixé, quand $u \rightarrow 0$, on a

(8.29) $\varphi \operatorname{Tr}_{s}\left[M_{3, u, T} \exp \left(-B_{3, u, T}^{2}\right)\right]$

$$
=\frac{2 C_{3,-1}(T)}{u T^{3}}-\left(C_{3,0}(T)+\varphi d^{S} \mu_{0}(T)\right)+O(u) .
$$

Preuve. - i) En utilisant les mêmes arguments que dans [BGS2, th. 2.11], on a $(8.28)$.

ii) En utilisant les mêmes arguments que dans [BGS2, th. 2.11], il existe $p^{\prime} \in \mathbb{N}$, des formes $\mu_{j}^{\prime}$ sur $S$ tels que, quand $u \rightarrow 0$, on a

$$
\varphi \operatorname{Tr}_{s}\left[M_{3, u, T} \exp \left(-B_{3, u, T}^{2}\right)\right]=\sum_{j=-p^{\prime}}^{k} \mu_{j}^{\prime} u^{j}+O\left(u^{k+1}\right) .
$$

En procédant comme en $[\mathrm{B} 1, \S 4]$, on a

$$
\lim _{u \rightarrow 0} \varphi \operatorname{Tr}_{s}\left[u M_{3, u, T} \exp \left(-B_{3, u, T}^{2}\right)\right]=2 \int_{Z} \frac{\widetilde{\omega}^{W}}{2 \pi T^{3}} \operatorname{Td}\left(T Z, g_{T}^{T Z}\right) \operatorname{ch}\left(\xi, h^{\xi}\right)
$$

et donc

$$
\mu_{j}^{\prime}=0 \quad \text { pour } \quad j \leq-2, \quad \mu_{-1}^{\prime}=\frac{2}{T^{3}} C_{3,-1}(T) .
$$

En utilisant (8.30) et (8.32), on sait que

$$
\mu_{0}^{\prime}=\lim _{u \rightarrow 0} \frac{\partial}{\partial u} \varphi \operatorname{Tr}_{s}\left[u M_{3, u, T} \exp \left(-B_{3, u, T}^{2}\right)\right] .
$$

De [BKö, th. 3.17 et 3.22], (8.28) et (8.33), on déduit que

$$
\mu_{0}^{\prime}=-\left(C_{3,0}(T)+\varphi d^{S} \mu_{0}(T)\right) .
$$

On a bien terminé la preuve de la proposition 8.6. BULLETIN DE LA SOCIÉTÉ MATHÉMATIQUE DE FRANCE 
Preuve du théorème 8.1. - D'après (8.29), on sait que pour $T$ fixé, quand $u \rightarrow 0$, on a

$$
\begin{aligned}
\varphi \operatorname{Tr}_{s}\left[M_{3, u^{2} / T^{2}, T}\right. & \left.\exp \left(-B_{3, u^{2} / T^{2}, T}^{2}\right)\right] \\
& =\frac{2}{u^{2} T} C_{3,-1}(T)-\left(C_{3,0}(T)+\varphi d^{S} \mu_{0}(T)\right)+O\left(u^{2}\right) .
\end{aligned}
$$

En comparant (8.15) et (8.35), on a

$$
a_{T, j}= \begin{cases}0 & \text { pour } j<-1 \\ 2 C_{3,-1}(T) & \text { pour } j=-1 \\ -T\left(C_{3,0}(T)+\varphi d^{S} \mu_{0}(T)\right) & \text { pour } j=0\end{cases}
$$

De $(8.15)$ et $(8.36)$, on tire le théorème 8.1.

d) L'asymptotique de $\mu_{0}(T)$ quand $T$ tend vers l'infini.

D'après (4.32), (8.17) et (8.36), on a

$$
\begin{aligned}
\lim _{T \rightarrow+\infty} T \varphi d^{S} \mu_{0}(T) & =-\lim _{T \rightarrow+\infty}\left(a_{T, 0}+T C_{3,0}(T)\right) \\
& =\int_{Y} \operatorname{Td}\left(T Y, g^{T Y}\right) \varphi_{1} \mathrm{~d}^{V} E_{1,0} .
\end{aligned}
$$

En utilisant [BGS2, prop. 2.10], et en procédant comme aux parties a), b), c) de ce paragraphe, on sait qu'il existe $C>0$, et des formes $b_{T, j}, \mathcal{C}^{\infty}$ sur $S$, qui dépendent continûment en $T \in[1,+\infty]$, tels que, pour $u \in] 0,1]$ et $T \in[1,+\infty[$,

$$
\begin{aligned}
\mid \operatorname{Tr}_{s}\left[M_{3, u^{2} / T^{2}, T} \exp \left(-B_{3, u^{2} / T^{2}, T}^{2}+d u\left(t \frac{\partial}{\partial t} B_{3, t, T}\right) \mid t=u^{2} / T^{2}\right)\right] & \\
& -\sum_{j=-n}^{0} \frac{b_{T, j}}{T} u^{2 j} \mid \leq C \frac{u^{2}}{T} .
\end{aligned}
$$

Plus précisément, pour $j \geq-n$, quand $T \rightarrow+\infty$, on a

$$
b_{T, j}=b_{\infty, j}+O\left(\frac{1}{T}\right) \text {. }
$$

En comparant (8.15), (8.28), (8.38), on a

$$
b_{T, 0}=\varphi^{-1} a_{T, 0}+T \mu_{0}(T) d u
$$

et donc

$$
\mu_{0}(T)=\frac{1}{T}\left[b_{\infty, 0}-\varphi^{-1} a_{\infty, 0}\right]^{d u}+O\left(\frac{1}{T^{2}}\right) .
$$

Par $(8.28),(8.37),(8.41)$, on a terminé la preuve du théorème 4.9. 


\section{Preuve du théorème 4.11}

Le but de ce paragraphe est de montrer le théorème 4.11. Pour montrer celuici, on s'inspire de [BerB, $\S 9]$, [B4, § 13] et [BL, § 13].

Ce paragraphe est organisé de la façon suivante. Dans a), on montre que la preuve du théorème 4.11 est locale sur $Y_{s_{0}}\left(s_{0} \in S\right)$. Dans b), étant donné $b_{0} \in Y_{s_{0}}$, on remplace $Z_{s_{0}} \operatorname{par}\left(T_{\mathbb{R}} Y\right)_{b_{0}} \times X_{b_{0}}$. On associe à cette dernière variété de nouveaux opérateurs $L_{3, \varepsilon, T}^{1}$ et $L_{3, \varepsilon, T}^{3}$. Dans c), on calcule l'asymptotique, quand $T$ tend vers l'infini, de la matrice de $L_{3, \varepsilon, T}^{3}$ relativement au scindement orthogonal de l'espace de Hilbert sur lequel l'opérateur $L_{3, \varepsilon, T}^{3}$ agit naturellement. Dans d), on introduit une famille de normes de Sobolev qui dépendent de $\varepsilon, T$. Ces normes de Sobolev prennent en compte la graduation des variables grassmanniennes dans $\Lambda\left(T_{\mathbb{R}}^{*} S\right)$. Dans e), on introduit un opérateur $\Xi_{\varepsilon}$ qui est un analogue d'un opérateur introduit dans [BerB, §9]. Dans f), on montre le théorème 9.2 .

On utilise les mêmes notations qu'aux $\S \S 4-8$.

\section{a) Localisation du problème.}

On utilise les mêmes notations qu'au $\S 8 \mathrm{a}$ ).

Proposition 9.1. - Il existe $\delta, c, C>0$ tels que, pour tout $0<\varepsilon \leq 1, T \geq 1$, on $a$

$$
\begin{aligned}
\mid \varphi \operatorname{Tr}_{s}\left[\frac{1}{\varepsilon} M_{3, \varepsilon^{2}, T / \varepsilon} \widetilde{G}_{\varepsilon}\left(B_{3, \varepsilon^{2}, T / \varepsilon}^{2}\right)\right] \\
\quad-\frac{2}{T} \varphi \operatorname{Tr}_{s}\left[\left(N_{X}-\operatorname{dim} X\right) \widetilde{G}_{\varepsilon}\left(B_{2, \varepsilon^{2}}^{2}\right)\right] \mid \leq \frac{C}{T^{1+\delta}} \exp \left(-\frac{C}{\varepsilon^{2}}\right) .
\end{aligned}
$$

Preuve. - Par (8.10), on a

$$
\operatorname{Tr}_{s}\left[\frac{1}{\varepsilon} M_{3, \varepsilon^{2}, T / \varepsilon} \widetilde{G}_{\varepsilon}\left(B_{3, \varepsilon^{2}, T / \varepsilon}^{2}\right)\right]=\psi_{\varepsilon} \operatorname{Tr}_{s}\left[\frac{1}{\varepsilon} C_{T / \varepsilon} M_{3,1, T / \varepsilon} C_{T / \varepsilon}^{-1} \widetilde{H}_{\varepsilon}\left(A_{T / \varepsilon}^{2}\right)\right] .
$$

En utilisant (5.2), (7.4), (8.12) et (8.13), en procédant comme au $\S \S 5 \mathrm{~g})$-i), on sait qu'il existe $\delta, C, c>0$ tels que, pour $0<\varepsilon \leq 1$ et $T \geq 1$

$$
\left\{\begin{array}{l}
\mid \operatorname{Tr}_{s}\left[\left(N_{X}-\operatorname{dim} X\right) \widetilde{H}_{\varepsilon}\left(A_{T / \varepsilon}^{2}\right)\right] \\
\quad-\operatorname{Tr}_{s}\left[\left(N_{X}-\operatorname{dim} X\right) \widetilde{H}_{\varepsilon}\left(B_{2,1}^{2}\right)\right] \mid \leq \frac{C}{T^{\delta}} \exp \left(-\frac{c}{\varepsilon^{2}}\right), \\
\left.\left.\quad-\frac{2}{T}\left(N_{X}-\operatorname{dim} X\right)\right) \widetilde{H}_{\varepsilon}\left(A_{T / \varepsilon}^{2}\right)\right] \mid \leq \frac{C}{T^{1+\delta}} \exp \left(-\frac{c}{\varepsilon^{2}}\right) .
\end{array}\right.
$$

De (9.2) et (9.3) on tire (9.1). 
D'après (9.1), il est clair que pour établir le théorème 4.11, il suffit d'établir le résultat suivant.

ThÉonème 9.2. - Pour $\alpha>0$ assez petit, il existe $\delta, c>0$ tels que, pour $0<\varepsilon \leq 1$ et $T \geq 1$,

$$
\begin{aligned}
\mid \varphi \operatorname{Tr}_{s}\left[\frac{1}{\varepsilon} M_{3, \varepsilon^{2}, T / \varepsilon} \widetilde{F}_{\varepsilon}\left(B_{3, \varepsilon^{2}, T / \varepsilon}^{2}\right)\right] & \\
& -\frac{2}{T} \varphi \operatorname{Tr}_{s}\left[\left(N_{X}-\operatorname{dim} X\right) \widetilde{F}_{\varepsilon}\left(B_{2, \varepsilon^{2}}^{2}\right)\right] \mid \leq \frac{C}{T^{1+\delta}} .
\end{aligned}
$$

Le reste du paragraphe est consacré à la preuve du théorème 9.2.

Par (7.2), on a

$$
\varphi \operatorname{Tr}_{s}\left[\frac{1}{\varepsilon} M_{3, \varepsilon^{2}, T / \varepsilon} \widetilde{F}_{\varepsilon}\left(B_{3, \varepsilon^{2}, T / \varepsilon}^{2}\right)\right]=\varphi \operatorname{Tr}_{s}\left[M_{3, \varepsilon, T}^{0} \widetilde{F}_{\varepsilon}\left(L_{3, \varepsilon, T}^{0}\right)\right] .
$$

Soit $\widetilde{F}_{\varepsilon}\left(L_{3, \varepsilon, T}^{0}\right)\left(x, x^{\prime}\right)\left(x, x^{\prime} \in Z_{s}\right)$ le noyau $\mathcal{C}^{\infty}$ de $\widetilde{F}_{\varepsilon}\left(L_{3, \varepsilon, T}^{0}\right)$ relativement à $\mathrm{d} v_{Z}\left(x^{\prime}\right) /(2 \pi)^{\operatorname{dim} Z}$. En utilisant la propriété de la vitesse finie de propagation, il est clair que pour $x \in Z_{s}, \widetilde{F}_{\varepsilon}\left(L_{3, \varepsilon, T}^{0}\right)(x, x)$ dépend seulement de la restriction de $L_{3, \varepsilon, T}^{0}$ à $\pi_{1}^{-1}\left(B^{Y}\left(\pi_{1} x, \alpha\right)\right)$.

b) Un nouvel opérateur sur $\left(T_{R} Y\right)_{b_{0}} \times X_{b_{0}}$.

Soit $g^{T S}$ une métrique sur $T S$. Elle induit naturellement une métrique $\operatorname{sur} \Lambda\left(T_{\mathbb{R}}^{*} S\right)$.

On fixe $b_{0} \in Y_{s_{0}}$ (avec $s_{0} \in S$ ). Sur $B^{Y}\left(b_{0}, r\right)$, on trivialise la fibration $\pi_{1}^{-1}\left(B^{Y}\left(b_{0}, r\right)\right) \rightarrow B^{Y}\left(b_{0}, r\right)$ et les fibrés vectoriels considérés comme au $\left.\S 7 \mathrm{~b}\right)$. Alors l'opérateur $L_{3, \varepsilon, T}^{0}$ agit sur l'espace vectoriel $H_{b_{0}}(r)$ des sections $\mathcal{C}^{\infty}$ de

$$
\Lambda\left(T_{\mathbb{R}}^{*} S\right)_{s_{0}} \widehat{\otimes} \pi_{1}^{*}\left(\Lambda\left(T^{*(0,1)} Y\right)\right)_{b_{0}} \widehat{\otimes}\left(\Lambda\left(T^{*(0,1)} X\right) \otimes \xi\right)_{\mid X_{b_{0}}}
$$

$\operatorname{sur} B^{Y}\left(b_{0}, r\right) \times X_{b_{0}}$.

Soit $G_{b_{0}}=\Omega\left(X_{b_{0}}, \xi_{\mid X_{b_{0}}}\right)$ l'espace vectoriel des sections de classe $\mathcal{C}^{\infty}$ de $\left(\Lambda\left(T^{*(0,1)} X\right) \otimes \xi\right)_{\mid X_{b_{0}}}$ sur $X_{b_{0}}$. Alors $G_{b_{0}}$ est muni d'une métrique hermitienne, et $\operatorname{Ker} D^{X}{ }_{\mid B^{Y}\left(b_{0}, r\right)}$ est un sous-fibré vectoriel $\mathbb{Z}$-gradué de $G_{b_{0}}$ sur $B^{Y}\left(b_{0}, r\right)$.

Pour $\alpha>0$ assez petit, il existe un fibré vectoriel $K \subset G_{b_{0}}, \mathbb{Z}$-gradué sur $\left(T_{\mathbb{R}} Y\right)_{b_{0}} \simeq \mathbb{R}^{2 m_{0}}$ qui coïncide avec Ker $D^{X}$ sur $B^{T Y}(0,2 \alpha)$, et avec Ker $D_{b_{0}}^{X}$ sur $\left(T_{\mathbb{R}} Y\right)_{b_{0}} \backslash B^{T Y}(0,3 \alpha)$, tel que si $K^{\perp}$ est le fibré vectoriel orthogonal à $K$ dans $G_{b_{0}}$, alors

$$
K^{\perp} \cap \operatorname{Ker} D_{b_{0}}^{X}=\{0\} .
$$

Soit $P_{b}$ (avec $b \in \mathbb{R}^{2 m_{0}}$ ) la projection orthogonale de $G_{b_{0}}$ sur $K_{b}$ pour le produit hermitien naturel sur $G_{b_{0}}$. On pose $P_{b}^{\perp}=1-P_{b}$.

TOME $127-1999-\mathrm{N}^{\circ} 4$ 
Soit $\varphi: \mathbb{R} \rightarrow[0,1]$ une fonction $\mathcal{C}^{\infty}$ telle que

$$
\varphi(t)= \begin{cases}1 & \text { pour }|t| \leq \alpha \\ 0 & \text { pour }|t| \geq 2 \alpha\end{cases}
$$

Soit $\Delta^{T Y}$ le Laplacien standard sur $\left(T_{\mathbb{R}} Y\right)_{b_{0}}$ correspondant à la métrique $g^{T Y_{b_{0}}}$. Soit $H_{b_{0}}$ l'espace vectoriel des sections $\mathcal{C}^{\infty}$ de

$$
\Lambda\left(T_{\mathbb{R}}^{*} S\right)_{s_{0}} \widehat{\otimes} \pi_{1}^{*}\left(\Lambda\left(T^{*(0,1)} Y\right)\right)_{b_{0}} \widehat{\otimes}\left(\Lambda\left(T^{*(0,1)} X\right) \otimes \xi\right)_{\mid X_{b_{0}}}
$$

$\operatorname{sur}\left(T_{\mathbb{R}} Y\right)_{b_{0}} \times X_{b_{0}}$. Soit $L_{3, \varepsilon, T}^{1}$ l'opérateur défini par

$$
L_{3, \varepsilon, T}^{1}=\varphi^{2}(|Y|) L_{3, \varepsilon, T}^{0}+\left(1-\varphi^{2}(|Y|)\right)\left(-\frac{1}{2} \varepsilon^{2} \Delta^{T Y}+T^{2} P_{Y}^{\perp} D_{b_{0}}^{X, 2} P_{Y}^{\perp}\right) .
$$

Alors $L_{3, \varepsilon, T}^{1}$ coïncide avec $L_{3, \varepsilon, T}^{0}$ sur $|Y| \leq \alpha$.

Pour $\varepsilon>0$, avec $s \in H_{b_{0}}$, on pose

$$
\left\{\begin{array}{l}
\left(S_{\varepsilon} s\right)(Y, x)=s(Y / \varepsilon, x), \quad \text { pour } \quad(Y, x) \in \mathbb{R}^{2 m_{0}} \times X_{b_{0}}, \\
L_{3, \varepsilon, T}^{2}=S_{\varepsilon}^{-1} L_{3, \varepsilon, T}^{1} S_{\varepsilon} .
\end{array}\right.
$$

On fait le même changement d'échelle sur les variables de Clifford $c\left(f_{\ell}\right)(1 \leq i \leq$ $\left.2 m_{0}\right)$ qu'au $\S 7$. On obtient ainsi un opérateur $L_{3, \varepsilon, T}^{3}$.

Soit $\widetilde{F}_{\varepsilon}\left(L_{3, \varepsilon, T}^{j}\right)\left(x, x^{\prime}\right)$ (avec $\left.x, x^{\prime} \in\left(T_{\mathbb{R}} Y\right)_{b_{0}} \times X_{b_{0}}\right)$ le noyau $\mathcal{C}^{\infty}$ de l'opérateur $\widetilde{F}_{\varepsilon}\left(L_{3, \varepsilon, T}^{j}\right)$ relativement à $1 /(2 \pi)^{\operatorname{dim} Z} \mathrm{~d} v_{T Y_{b_{0}}} \mathrm{~d} v_{X_{b_{0}}}$. D'après la propriété de vitesse finie de propagation, on sait que si $x \in X_{b_{0}}$ est identifié à $(0, x)$, alors on a

$$
\widetilde{F}_{\varepsilon}\left(L_{3, \varepsilon, T}^{0}\right)(x, x)=\widetilde{F}_{\varepsilon}\left(L_{3, \varepsilon, T}^{1}\right)(x, x) .
$$

Soit $E^{0}$ l'espace vectoriel des sections de carré intégrable de

$$
\Lambda\left(T_{\mathbb{R}}^{*} S\right)_{s_{0}} \widehat{\otimes} \pi_{1}^{*}\left(\Lambda\left(T^{*(0,1)} Y\right)\right)_{b_{0}} \widehat{\otimes}\left(\Lambda\left(T^{*(0,1)} X\right) \otimes \xi\right)_{\mid X_{b_{0}}}
$$

$\operatorname{sur}\left(T_{\mathbb{R}} Y\right)_{b_{0}} \times X_{b_{0}}$.

Soit $F_{\varepsilon}^{0}$ l'espace vectoriel des sections de carré intégrable de

$$
\Lambda\left(T_{\mathbb{R}}^{*} S\right)_{s_{0}} \widehat{\otimes} \pi_{1}^{*}\left(\Lambda\left(T^{*(0,1)} Y\right)\right)_{b_{0}} \widehat{\otimes} S_{\varepsilon}^{-1 *} K
$$

$\operatorname{sur}\left(T_{\mathbb{R}} Y\right)_{b_{0}}$. Alors l'espace $F_{\varepsilon}^{0}$ a un sens pour $\varepsilon=0$, et $F_{0}^{0}$ est l'espace vectoriel des sections de carré intégrable de

$$
\Lambda\left(T_{\mathbb{R}}^{*} S\right)_{s_{0}} \widehat{\otimes} \pi_{1}^{*}\left(\Lambda\left(T^{*(0,1)} Y\right)\right)_{b_{0}} \widehat{\otimes} \operatorname{Ker} D_{b_{0}}^{X}
$$

$\operatorname{sur}\left(T_{\mathbb{R}} Y\right)_{b_{0}}$. L'espace $F_{\varepsilon}^{0}$ est un sous-espace de Hilbert de $E^{0}$. Soit $F_{\varepsilon}^{0, \perp}$ l'espace orthogonal à $F_{\varepsilon}^{0}$ dans $E^{0}$. Soit $p_{\varepsilon}$ la projection orthogonale de $E^{0} \operatorname{sur} F_{\varepsilon}^{0}$. 
On pose $p_{\varepsilon}^{\perp}=1-p_{\varepsilon}$. Pour $s \in E^{0}, Y \in T_{\mathbb{R}} Y$, on a

$$
p_{\varepsilon} s(Y)=P_{\varepsilon Y} s(Y, \cdot) \text {. }
$$

On pose

$$
\begin{cases}E_{\varepsilon, T}=p_{\varepsilon} L_{3, \varepsilon, T}^{3} p_{\varepsilon}, & F_{\varepsilon, T}=p_{\varepsilon} L_{3, \varepsilon, T}^{3} p_{\varepsilon}^{\perp}, \\ G_{\varepsilon, T}=p_{\varepsilon}^{\perp} L_{3, \varepsilon, T}^{3} p_{\varepsilon}, & H_{\varepsilon, T}=p_{\varepsilon}^{\perp} L_{3, \varepsilon, T}^{3} p_{\varepsilon}^{\perp} .\end{cases}
$$

Alors on écrit $L_{3, \varepsilon, T}^{3}$ sous forme matricielle relativement au scindage $E^{0}=$ $F_{\varepsilon}^{0} \oplus F_{\varepsilon}^{0, \perp}$ :

$$
L_{3, \varepsilon, T}^{3}=\left[\begin{array}{ll}
E_{\varepsilon, T}, & F_{\varepsilon, T} \\
G_{\varepsilon, T}, & H_{\varepsilon, T}
\end{array}\right] .
$$

\section{c) L'asymptotique de la matrice $L_{3, \epsilon, T}^{3}$ quand $T$ tend vers l'infini.}

On rappelle que ${ }^{0} \nabla^{\Omega(Z, \xi \mid Z)}$ est la connexion sur $\Omega\left(Z, \xi_{\mid Z}\right)$ définie en (5.13).

Si $C$ est une section de $\pi_{2}^{*} \Lambda\left(T_{\mathbb{R}}^{*} S\right) \widehat{\otimes} c\left(T_{\mathbb{R}} Y\right)_{b_{0}}$, et si $b \in Y_{s_{0}}$ est un point près de $b_{0}$, soit $C_{\varepsilon}^{3}(b)$ l'élément de $\pi_{2}^{*} \Lambda\left(T_{\mathbb{R}}^{*} S\right) \widehat{\otimes} \operatorname{End}\left(\Lambda\left(T_{\mathbb{R}}^{*} Y\right)\right)_{b_{0}}$ qui est obtenu en utilisant la trivialisation de $\pi_{2}^{*} \Lambda\left(T_{\mathbb{R}}^{*} S\right) \widehat{\otimes} \operatorname{End}\left(\Lambda\left(T_{\mathbb{R}}^{*} Y\right)\right)$, relativement à la connexion $\psi_{\varepsilon}{ }^{\prime} \nabla^{\pi_{2}^{*} \Lambda\left(T_{\mathbb{R}}^{*} S\right) \widehat{\otimes} \Lambda\left(T^{*(0,1)} Y\right)} \psi_{\varepsilon}^{-1}$ comme au $\left.\S 7 \mathrm{~b}\right)$, et par le changement d'échelle sur les éléments $c\left(T_{\mathbb{R}} Y\right)_{b_{0}}$ défini à la définition 7.2 .

Dans la suite, on note $[A, B]+$ l'anti-commutateur de $A$ et $B$. On pose :

$$
L_{1}^{\prime \xi}=L^{\xi}+\frac{1}{2} \operatorname{Tr}\left[R^{T X}\right] .
$$

ThÉORÈme 9.3. - Il existe des opérateurs $E_{\varepsilon}, F_{\varepsilon}, G_{\varepsilon}, H_{\varepsilon}$ tels que quand $T$ tend vers l'infini,

$$
\begin{cases}E_{\varepsilon, T}=E_{\varepsilon}+O\left(\frac{1}{T}\right), & F_{\varepsilon, T}=T F_{\varepsilon}+O(1), \\ G_{\varepsilon, T}=T G_{\varepsilon}+O(1), & H_{\varepsilon, T}=T^{2} H_{\varepsilon}+O(T) .\end{cases}
$$

On pose :

$$
\begin{aligned}
Q_{\varepsilon}=\varphi^{2}(\varepsilon|Y|)\left\{-\frac{1}{2}\left[\nabla_{e_{i}}^{\Lambda\left(T^{*(0,1)} X\right) \otimes \xi},\right.\right. \\
\frac{1}{2}\left\langle A\left(e_{i}\right) f_{\ell, 1}^{H}, e_{j}\right\rangle_{g^{T Y}}\left\{\varepsilon c\left(f_{\ell, 1}^{H}\right)\right\}_{\varepsilon}^{3} c\left(e_{j}\right) \\
\left.+\left\langle S_{1}\left(e_{i}\right) g_{\alpha, 3, \infty}^{H}, e_{j}\right\rangle_{g^{T X}} g^{\alpha} \frac{c\left(e_{j}\right)}{\sqrt{2}}\right]_{+} \\
+\frac{1}{2}\left\{\varepsilon c\left(f_{\ell, 1}^{H}\right)\right\}_{\varepsilon}^{3} c\left(e_{j}\right) L_{1}^{\prime \xi}\left(f_{\ell, 1}^{H}, e_{j}\right) \\
\left.+\frac{1}{\sqrt{2}} g^{\alpha} c\left(e_{j}\right) L_{1}^{\prime \xi}\left(g_{\alpha, 3, \infty}^{H}, e_{j}\right)\right\}_{\varepsilon Y} .
\end{aligned}
$$

TOME $127-1999-\mathrm{N}^{\circ} 4$ 
Alors on a $Q_{\varepsilon}\left(F_{\varepsilon}^{0}\right) \subset F_{\varepsilon}^{0, \perp}$, et

$$
\left\{\begin{array}{l}
F_{\varepsilon}=p_{\varepsilon} Q_{\varepsilon} p_{\varepsilon}^{\perp}, \quad G_{\varepsilon}=p_{\varepsilon}^{\perp} Q_{\varepsilon} p_{\varepsilon}, \\
H_{\varepsilon}=p_{\varepsilon}^{\perp}\left(\varphi^{2}(\varepsilon|Y|) D_{\varepsilon Y}^{X, 2}+\left(1-\varphi^{2}(\varepsilon|Y|)\right) D_{b_{0}}^{X, 2}\right) p_{\varepsilon}^{\perp} .
\end{array}\right.
$$

Preuve. - Par (7.8), on voit facilement que le coefficient de $T^{2}$ dans l'expansion asymptotique de $L_{3, \varepsilon, T}^{3}$ est $H_{\varepsilon}$.

En utilisant (1.35) et (7.8), on voit que le coefficient de $T$ dans l'expansion de $L_{3, \varepsilon, T}^{3}$ est l'opérateur $Q_{\varepsilon}$.

D'après le même calcul que dans [BerB, théorème 9.3], on a

$$
\begin{aligned}
& {\left[D^{X}, D_{\infty}^{H}+{ }^{0} \nabla^{\Omega(Z, \xi \mid Z)}\right]} \\
& =-\frac{1}{2}\left[\nabla_{e_{i}}^{\Lambda\left(T^{*(0,1)} X\right) \otimes \xi}, \frac{1}{2}\left\langle A\left(e_{i}\right) f_{\ell, 1}^{H}, e_{j}\right\rangle_{g^{T X}} c\left(f_{\ell, 1}^{H}\right) c\left(e_{j}\right)\right. \\
& \left.\quad+\left\langle S_{1}\left(e_{i}\right) g_{\alpha, 3, \infty}^{H}, e_{j}\right\rangle_{g^{T X}} g^{\alpha} c\left(e_{j}\right) / \sqrt{2}\right]_{+} \\
& \quad+\frac{1}{2} c\left(f_{\ell, 1}^{H}\right) c\left(e_{j}\right) L_{1}^{\prime \xi}\left(f_{\ell, 1}^{H}, e_{j}\right) \\
& +(1 / \sqrt{2}) g^{\alpha} c\left(e_{j}\right) L_{1}^{\prime \xi}\left(g_{\alpha, 3, \infty}^{H}, e_{j}\right) .
\end{aligned}
$$

Par (9.12), (9.16) et (9.18), il est clair que $Q_{\varepsilon}\left(F_{\varepsilon}^{0}\right) \subset F_{\varepsilon}^{0, \perp}$.

On a bien terminé la preuve du théorème 9.3 .

Il est clair que pour $Y \in\left(T_{\mathbb{R}} Y\right)_{b_{0}}, H_{\varepsilon Y}$ est un opérateur elliptique agissant le long de la fibre $X_{b_{0}}$.

Proposition 9.4. - Pour tout $\varepsilon>0$

$$
\operatorname{Ker} H_{\varepsilon Y}=\Lambda\left(T_{\mathbb{R}}^{*} S\right)_{s_{0}} \widehat{\otimes} \Lambda\left(T_{\mathbb{R}}^{*} Y\right)_{b_{0}} \widehat{\otimes} K_{\varepsilon Y} .
$$

Preuve. - La preuve est la même que dans [BerB, prop. 9.4].

\section{d) Une famille d'espaces de Sobolev.}

Soit

$$
g_{\varepsilon}(Y)=1+\left(1+|Y|^{2}\right) \varphi^{2}\left(\frac{1}{2} \varepsilon|Y|\right) .
$$

Pour $0 \leq q \leq 2 \operatorname{dim} V$, soit $E_{q}^{0}$ l'espace vectoriel des sections de carré intégrable de

$$
\bigoplus_{q_{1}+q_{2}=q} \Lambda^{q_{1}}\left(T_{\mathbb{R}}^{*} S\right) \widehat{\otimes} \Lambda^{q_{2}}\left(T_{\mathbb{R}}^{*} Y\right)_{b_{0}} \widehat{\otimes}\left(\Lambda\left(T^{*(0,1)} X\right) \otimes \xi\right)_{\mid X_{b_{0}}}
$$

BULLETIN DE LA SOCIÉTÉ MATHÉMATIQUE DE FRANCE 
$\operatorname{sur}\left(T_{\mathbb{R}} Y\right)_{b_{0}} \times X_{b_{0}}$. Alors

$$
E^{0}=\bigoplus_{q=0}^{2 \operatorname{dim} V} E_{q}^{0}
$$

Pour $p \in \mathbb{R}$, on note $E^{p}$ et $E_{q}^{p}$ les espaces de Sobolev d'ordre $p$ correspondants.

Pour $s \in E_{q}^{0}$, on pose

$$
|s|_{\varepsilon, 0}^{2}=\int_{\left(T_{\mathbb{R}} Y\right)_{b_{0}} \times X_{b_{0}}}|s(Y, x)|^{2} g_{\varepsilon}^{2(2 \operatorname{dim} V-q)}(Y) \frac{\mathrm{d} v_{T Y_{b_{0}}}(Y) \mathrm{d} v_{X_{b_{0}}}(x)}{(2 \pi)^{\operatorname{dim} Z}}
$$

Soit $\langle,\rangle_{\varepsilon, 0}$ le produit hermitien sur $E^{0}$ correspondant à ||$_{\varepsilon, 0}$. Pour $s \in E^{1}$, on pose

$$
\begin{aligned}
|s|_{\varepsilon, T, 1}^{2}=T^{2}\left|P_{\varepsilon Y}^{\perp} s\right|_{\varepsilon, 0}^{2} & +\left|P_{\varepsilon Y} s\right|_{\varepsilon, 0}^{2} \\
& +\sum_{1}^{2 m}\left|\nabla_{f_{\alpha}} s\right|_{\varepsilon, 0}^{2}+T^{2} \sum\left|\nabla_{e_{i}}^{\Lambda\left(T^{*(0,1)} X\right) \otimes \xi} P_{\varepsilon Y}^{\perp} s\right|_{\varepsilon, 0}^{2} .
\end{aligned}
$$

Alors (9.22) définit une norme sur $E^{1}$. Soit $E^{-1}$ l'antidual de $E^{1}$, et soit ||$_{\varepsilon, T,-1}$ la norme sur $E^{-1}$ associée à la norme $\left.\right|_{\varepsilon, T, 1} \operatorname{sur} E^{1}$. On identifie $E^{0}$ à son antidual par $\langle,\rangle_{\varepsilon, 0}$.

Par le théorème 9.3, il est clair que l'argument d'analyse fonctionnelle de [BL, $\S 13(\mathrm{k})-(\mathrm{o})]$ et [BerB, $\S 9(\mathrm{~d})]$ peut être utilisé directement dans notre situation. En effet, la structure asymptotique de $L_{3, \varepsilon, T}^{3}$ quand $T \rightarrow+\infty$ est la même que dans [BerB, $\S 9(\mathrm{c})]$. Bien sûr, on a aussi des variables grassmanniennes supplémentaires $g^{\alpha} \in T_{\mathbb{R}}^{*} S$, mais elles sont du même type que les $f^{\ell}$.

\section{e) Un opérateur $\boldsymbol{\Psi}_{\boldsymbol{\epsilon}}$.}

Soit $F_{\varepsilon}$ l'espace vectoriel des sections $\mathcal{C}^{\infty}$ de $\Lambda\left(T_{\mathbb{R}}^{*} S\right)_{s_{0}} \widehat{\otimes} \Lambda\left(T_{\mathbb{R}}^{*} Y\right)_{b_{0}} \widehat{\otimes} S_{\varepsilon}^{-1 *} K$ $\operatorname{sur}\left(T_{\mathbb{R}} Y\right)_{b_{0}}$. Alors $H_{\varepsilon}$ est inversible sur $F_{\varepsilon}^{0, \perp}$.

DÉfinition 9.5. - Soit $\Psi_{\varepsilon}$ l'opérateur de $F_{\varepsilon}$ dans $F_{\varepsilon}$ défini par

$$
\Psi_{\varepsilon}=E_{\varepsilon}-F_{\varepsilon} H_{\varepsilon}^{-1} G_{\varepsilon} .
$$

On vérifie facilement que l'opérateur $\Psi_{\varepsilon}$ est un opérateur elliptique d'ordre 2 agissant $\operatorname{sur} F_{\varepsilon}$.

Pour $U \in B^{T Y}(0,2 \alpha)$, on identifie $\left(\pi_{2}^{*} \Lambda\left(T_{\mathbb{R}}^{*} S\right) \widehat{\otimes} \Lambda\left(T^{*(0,1)} Y\right)\right)_{U}$ à $\pi_{2}^{*} \Lambda\left(T_{\mathbb{R}}^{*} S\right)_{s_{0}}$ $\widehat{\otimes} \Lambda\left(T^{*(0,1)} Y\right)_{b_{0}}$ par transport parallèle le long de la géodésique dans $Y$, $t \in[0,1] \rightarrow t U$, relativement à la connexion

$$
\psi_{\varepsilon}{ }^{\prime} \nabla^{\pi_{2}^{*} \Lambda\left(T_{\mathbb{R}}^{*} S\right) \widehat{\otimes} \Lambda\left(T^{*(0,1)} Y\right)} \psi_{\varepsilon}^{-1}
$$

TOME $127-1999-\mathrm{N}^{\circ} 4$ 
L'opérateur $B_{2, \varepsilon^{2}}^{2}$ agit sur les sections $\mathcal{C}^{\infty}$ de $\Lambda\left(T_{\mathbb{R}}^{*} S\right)_{s_{0}} \widehat{\otimes} \Lambda\left(T^{*(0,1)} Y\right)_{b_{0}} \widehat{\otimes} K$ sur $B^{T Y}(0,2 \alpha)$. Si l'on fait le même changement de coordonnées locales sur $Y_{s}$, et le même changement d'échelle sur les variables de Clifford $c\left(f_{\ell}\right)$ qu'au $\S 9 \mathrm{~b}$ ), alors, à partir de $B_{2, \varepsilon^{2}}^{2}$, on obtient un opérateur $\Sigma_{\varepsilon}^{3}$, qui agit sur les sections $\mathcal{C}^{\infty}$ $\operatorname{de} \Lambda\left(T_{\mathbb{R}}^{*} S\right)_{s_{0}} \widehat{\otimes} \Lambda\left(T_{\mathbb{R}}^{*} Y\right)_{b_{0}} \widehat{\otimes} S_{\varepsilon}^{-1 *} K \operatorname{sur} B^{T Y}(0,2 \alpha / \varepsilon)$.

ThÉORÈme 9.6. - Sur $B^{T Y}(0,2 \alpha / \varepsilon)$, on a

$$
\Psi_{\varepsilon}=\Sigma_{\varepsilon}^{3} .
$$

Preuve. - En utilisant (7.8) et en procédant comme en [BL, th. 13.43], on a $(9.24)$.

\section{f) Preuve du théorème 9.2.}

En utilisant (7.7), (7.15), les théorèmes 9.3 et 9.6 , la preuve du théorème 9.2 est la même que dans [BL, $\S 13(\mathrm{q})]$ et $[\mathrm{BerB}, \S 9(\mathrm{~g})]$.

\section{La classe de Bott-Chern $\widetilde{\operatorname{ch}}\left(E_{2}, H\left(Z, \xi_{\mid Z}\right), h^{E_{2}}, h^{H(Z, \xi \mid Z)}\right)$}

Le but de ce paragraphe est de construire la classe de Bott-Chern $\widetilde{c h}\left(E_{2}\right.$, $\left.H\left(Z, \xi_{\mid Z}\right), h^{E_{2}}, h^{H(Z, \xi \mid Z)}\right) \in P^{S} / P^{S, 0}$, et de démontrer les théorèmes 3.8 et 3.9 .

Ce paragraphe est organisé de la façon suivante. Dans a), on généralise les résultats de [BGS1] sur les classes de Bott-Chern. Dans b), on rappelle la propriété de fonctorialité de la suite spectrale de Leray [Grot]. Dans c), d'après b), on construit un bicomplexe de fibrés holomorphes sur $S$ qui calcule la suite spectrale de Leray. Dans d), on construit la classe de Bott-Chern $\widetilde{\mathrm{ch}}\left(E_{2}, H\left(Z, \xi_{\mid Z}\right), h^{E_{2}}, h^{H(Z, \xi \mid Z)}\right) \in P^{S} / P^{S, 0}$ et on démontre le théorème 3.8 . Dans e), on montre le théorème 3.9 .

On suppose ici que $\pi_{1}$ est projective, et que $V$ et $W$ sont des variétés projectives. On utilise les mêmes notations qu'au $\S 3$.

\section{a) Classes de Bott-Chern.}

Soit $(E, v)=\left(E^{i}, v\right)_{0 \leq i \leq n}$ un complexe holomorphe de fibrés vectoriels holomorphes sur une variété complexe $S$. Pour $s \in S$, on note $H_{s}^{i}(E)$ la $i$-ième cohomologie du complexe $(E, v)_{s}$. On suppose que pour $0 \leq i \leq n$, le rang des fibres $H^{i}(E)$ est localement constant; alors, $H^{i}(E)$ est un fibré vectoriel holomorphe sur $S$. Soient $F^{i}=v\left(E^{i-1}\right)$ et $G^{i}=\operatorname{Ker} v_{\mid E^{i}}$.

On a les suites exactes suivantes :

$$
\left\{\begin{array}{l}
0 \rightarrow G^{i} \longrightarrow E^{i} \longrightarrow F^{i+1} \rightarrow 0, \\
0 \rightarrow F^{i} \longrightarrow G^{i} \longrightarrow H^{i}(E) \rightarrow 0 .
\end{array}\right.
$$


Soient $h^{E^{i}}, h^{H^{i}(E)}$ des métriques hermitiennes sur $E^{i}, H^{i}(E)$. On note

$$
h^{E}=\bigoplus_{i} h^{E^{i}}, \quad h^{H(E)}=\bigoplus_{i} h^{H^{i}(E)}
$$

des métriques sur $E=\bigoplus_{i} E^{i}, H(E)=\bigoplus_{i} H^{i}(E)$. Soient $h^{F^{i}}$ et $h^{G^{i}}$ les métriques sur $F^{i}$ et $G^{i}$ induites par $h^{E^{i}}$.

On note

$$
\widetilde{\operatorname{ch}}\left(E^{i}, F^{i+1}, h^{E^{i}}, h^{F^{i+1}}\right), \quad \widetilde{\operatorname{ch}}\left(G^{i}, H^{i}(E), h^{G^{i}}, h^{H^{i}(E)}\right) \in P^{S} / P^{S, 0}
$$

les classes de Bott-Chern associées à (10.1) [BGS1, §1(f)]. On pose

$$
\left\{\begin{array}{l}
\operatorname{ch}\left(E, h^{E}\right)=\sum_{i=0}^{n}(-1)^{i} \operatorname{ch}\left(E^{i}, h^{E^{i}}\right) \\
\operatorname{ch}\left(H(E), h^{H(E)}\right)=\sum_{i=0}^{n}(-1)^{i} \operatorname{ch}\left(H^{i}(E), h^{H^{i}(E)}\right) .
\end{array}\right.
$$

On dit que $E$ est scindé, si

$$
E^{i}=G^{i} \oplus F^{i+1}, \quad G^{i}=H^{i}(E) \oplus F^{i}
$$

et si les scindements (10.3) sont orthogonaux.

Proposition 10.1. - Il existe une unique manière de définir une classe de Bott-Chern $\widetilde{\operatorname{ch}}\left(E, H(E), h^{E}, h^{H(E)}\right) \in P^{S} / P^{S, 0}$ telle que :

i) On $a$

$$
\frac{\bar{\partial} \partial}{2 i \pi} \widetilde{\operatorname{ch}}\left(E, H(E), h^{E}, h^{H(E)}\right)=\operatorname{ch}\left(H(E), h^{H(E)}\right)-\operatorname{ch}\left(E, h^{E}\right)
$$

ii) Pour tout application holomorphe de variétés complexes $f: S^{\prime} \rightarrow S$,

$$
\widetilde{\operatorname{ch}}\left(f^{*} E, f^{*} H(E), h^{f^{*} E}, h^{f^{*} H(E)}\right)=f^{*} \widetilde{\operatorname{ch}}\left(E, H(E), h^{E}, h^{H(E)}\right) .
$$

iii) Si E est scindé, alors

$$
\widetilde{\operatorname{ch}}\left(E, H(E), h^{E}, h^{H(E)}\right)=0 \quad \text { dans } \quad P^{S} / P^{S, 0} .
$$

Preuve. - La preuve est la même que dans [BGS1, théorème 1.29]. 
Proposition 10.2. - On a dans $P^{S} / P^{S, 0}$

$$
\begin{aligned}
& \widetilde{\operatorname{ch}}\left(E, H(E), h^{E}, h^{H(E)}\right) \\
& \quad=\sum_{i}(-1)^{i}\left[\widetilde{\operatorname{ch}}\left(G^{i}, H^{i}(E), h^{G^{i}}, h^{H^{i}(E)}\right)+\widetilde{\operatorname{ch}}\left(E^{i}, F^{i+1}, h^{E^{i}}, h^{F^{i+1}}\right)\right] .
\end{aligned}
$$

Preuve. - On vérifie facilement que le terme à droite de (10.7) vérifie (10.4)(10.6). D'après la proposition 10.1 , on a (10.7).

Par la théorie de Hodge, on peut identifier $H(E)$ au sous-fibré de $E$ formé des éléments harmoniques dans $E$. Soit $h^{H(E)}$ la métrique induite par la métrique $h^{E}$ sur $H(E)$. Soient $\nabla^{E}, \nabla^{H(E)}$ les connexions holomorphes hermitiennes sur $\left(E, h^{E}\right),\left(H(E), h^{H(E)}\right)$. Soit $N$ l'opérateur de nombre sur $E$. Soit $v^{*}$ l'adjoint de $v$ pour $h^{E}$. On pose $V=v+v^{*}$. Pour $u \geq 0$, soit $A_{u}$ la superconnexion

$$
A_{u}=\nabla^{E}+\sqrt{u} V \text {. }
$$

Pour $s \in \mathbb{C}$ et $\operatorname{Re} s>1$, on pose

$$
\begin{aligned}
\zeta_{1}(s)=-\frac{1}{\Gamma(s)} \int_{0}^{1} u^{s-1}\left\{\varphi \operatorname{Tr}_{s}\left[N \exp \left(-A_{u}^{2}\right)\right]\right. \\
\left.-\varphi \operatorname{Tr}_{s}\left[N \exp \left(-\nabla^{H(E), 2}\right)\right]\right\} \mathrm{d} u
\end{aligned}
$$

Alors $\zeta_{1}(s)$ se prolonge en une fonction holomorphe de $s \in \mathbb{C}$ près de $s=0$.

Pour $s \in \mathbb{C}$, $\operatorname{Re} s<\frac{1}{2}$, on pose

$$
\begin{aligned}
\zeta_{2}(s)=-\frac{1}{\Gamma(s)} \int_{1}^{+\infty} u^{s-1}\left\{\varphi \operatorname{Tr}_{s}[\right. & \left.\exp \left(-A_{u}^{2}\right)\right] \\
& \left.-\varphi \operatorname{Tr}_{s}\left[N \exp \left(-\nabla^{H(E), 2}\right)\right]\right\} \mathrm{d} u .
\end{aligned}
$$

En utilisant $\left[\mathrm{BeGeV}\right.$, th. 9.2], $\zeta_{2}(s)$ est une fonction holomorphe de $s$.

DÉFINITION 10.3. - On pose

$$
\zeta\left(E, h^{E}\right)=\frac{\partial}{\partial s}\left(\zeta_{1}+\zeta_{2}\right)(0)
$$

Alors $\zeta\left(E, h^{E}\right)$ est une forme $\mathcal{C}^{\infty}$ sur $S$. En utilisant $(10.9),(10.10)$, on a

$$
\begin{aligned}
& \zeta\left(E, h^{E}\right)=-\int_{0}^{1}\left\{\varphi \operatorname{Tr}_{s}\left[N \exp \left(-A_{u}^{2}\right)\right]-\varphi \operatorname{Tr}_{s}\left[N \exp \left(-\nabla^{E, 2}\right)\right]\right\} \frac{\mathrm{d} u}{u} \\
& -\int_{1}^{+\infty}\left\{\varphi \operatorname{Tr}_{s}\left[N \exp \left(-A_{u}^{2}\right)\right]-\varphi \operatorname{Tr}_{s}\left[N \exp \left(-\nabla^{H(E), 2}\right)\right]\right\} \frac{\mathrm{d} u}{u} \\
& +\Gamma^{\prime}(1)\left\{\varphi \operatorname{Tr}_{s}\left[N \exp \left(-\nabla^{E, 2}\right)\right]-\varphi \operatorname{Tr}_{s}\left[N \exp \left(-\nabla^{H(E), 2}\right)\right]\right\} .
\end{aligned}
$$


Par [BGS1, th. 1.15], les formes $\zeta\left(E, h^{E}\right)$ sont dans $P^{S}$, et on a

$$
\frac{\bar{\partial} \partial}{2 i \pi} \zeta\left(E, h^{E}\right)=\operatorname{ch}\left(H(E), h^{H(E)}\right)-\operatorname{ch}\left(E, h^{E}\right) .
$$

Proposition 10.4. - On a l'identité

$$
\zeta\left(E, h^{E}\right)=\widetilde{\operatorname{ch}}\left(E, H(E), h^{E}, h^{H(E)}\right) \quad \text { dans } \quad P^{S} / P^{S, 0} .
$$

Démonstration. - La preuve est la même que dans [BGS1, cor. 1.30].

Proposition 10.5. - Soit $E=E^{0} \supset \cdots \supset E^{m}=0$ une filtration de fibrés vectoriels holomorphes sur $S$. On pose $\mathrm{Gr}^{i} E=E^{i} / E^{i+1}$. Soient $h^{E}$, $h^{\mathrm{Gr} E}=\oplus h^{\mathrm{Gr}^{i} E}$ des métriques hermitiennes sur $E, \operatorname{Gr} E=\bigoplus \mathrm{Gr}^{i} E$. Soit $h^{E^{i}}$ la métrique sur $E^{i}$ induite par $h^{E}$. Alors il existe une unique manière de définir une classe de Bott-Chern $\widetilde{\operatorname{ch}}\left(E, \operatorname{Gr} E, h^{E}, h^{\mathrm{Gr} E}\right)$ appartenant à $P^{S} / P^{S, 0}$ telle que :

i) On $a$

$$
\frac{\bar{\partial} \partial}{2 i \pi} \widetilde{\operatorname{ch}}\left(E, \operatorname{Gr} E, h^{E}, h^{\mathrm{Gr} E}\right)=\operatorname{ch}\left(\operatorname{Gr} E, h^{\mathrm{Gr} E}\right)-\operatorname{ch}\left(E, h^{E}\right) .
$$

ii) Pour toute application holomorphe de variétés complexes $f: S^{\prime} \rightarrow S$,

$$
\widetilde{\operatorname{ch}}\left(f^{*} E, f^{*} \operatorname{Gr} E, h^{f^{*} E}, h^{f^{*} \mathrm{Gr} E}\right)=f^{*} \widetilde{\operatorname{ch}}\left(E, \operatorname{Gr} E, h^{E}, h^{\mathrm{Gr} E}\right) \text {. }
$$

iii) $S i\left(E^{k}, h^{E^{k}}\right)=\bigoplus_{i \geq k}\left(\mathrm{Gr}^{i} E, h^{\mathrm{Gr}^{i} E}\right)$ pour tout $k \geq 0$, alors

$$
\widetilde{\operatorname{ch}}\left(E, \operatorname{Gr} E, h^{E}, h^{\mathrm{Gr} E}\right)=0 .
$$

Preuve. - La preuve est la même que dans [BGS1, th. 1.29].

Remarque 10.6. - On a les suites exactes suivantes

$$
S_{i}: 0 \rightarrow E^{i+1} \longrightarrow E^{i} \longrightarrow \mathrm{Gr}^{i} E \rightarrow 0
$$

D'après la proposition 10.5. on vérifie facilement qu'on a dans $P^{S} / P^{S, 0}$

$$
\widetilde{\operatorname{ch}}\left(E, \operatorname{Gr} E, h^{E}, h^{\mathrm{Gr} E}\right)=-\sum_{i=0}^{m} \widetilde{\operatorname{ch}}\left(E^{i}, \mathrm{Gr}^{i} E, h^{E^{i}}, h^{\mathrm{Gr}^{i} E}\right)
$$


Soit $\mathcal{E}=\left(\mathcal{E}^{p, q}\right)$ (où $0 \leq p, q \leq n$ ) un bicomplexe de fibrés vectoriels holomorphes hermitiens sur $S$. Soit $H(\mathcal{E})$ la cohomologie de $\mathcal{E}$. Soit $\left(\mathcal{E}_{r}, d_{r}\right)$ la suite spectrale induite par la filtration $F^{p} \mathcal{E}=\bigoplus_{p^{\prime} \geq p} \mathcal{E}^{p^{\prime}} \cdot \bullet$ Soit $F H(\mathcal{E})$ la filtration associée sur $H(\mathcal{E})$. Alors $\mathcal{E}_{\infty}=\operatorname{Gr} H(\mathcal{E})$ (cf. [GrH, § 3.5]).

On suppose que pour $p, q, r \geq 0$, le rang des fibres $\mathcal{E}_{r}^{p, q}$ est localement constant.

Soit $h^{\mathcal{E}}=\bigoplus h^{\mathcal{E}^{p, q}}$ une métrique sur $\mathcal{E}=\bigoplus \mathcal{E}^{p, q}$ et $h^{\mathcal{E}_{0}}$ la métrique correspondante sur $\mathcal{E}_{0}=\mathcal{E}$. Soient $h^{\mathcal{E}_{r}}(r \geq 1)$ et $h^{H(\mathcal{E})}$ des métriques sur $\mathcal{E}_{r}$ et $H(\mathcal{E})$. On suppose que $h^{\mathcal{E}_{r}}=h^{\mathcal{E}_{\infty}}$ pour $r>n$. On pose

$(10.20) \widetilde{\operatorname{ch}}\left(H(\mathcal{E}), \mathcal{E}_{\infty}, h^{H(\mathcal{E})}, h^{\mathcal{E}_{\infty}}\right)=\sum_{k=0}^{n}(-1)^{k} \tilde{\operatorname{ch}}\left(H^{k}(\mathcal{E}), \operatorname{Gr} H^{k}(\mathcal{E}), h^{H^{k}(\mathcal{E})}, h^{\mathcal{E}_{\infty}}\right)$

ThÉORÈme 10.7. - On a dans $P^{S} / P^{S, 0}$

$$
\begin{aligned}
& \widetilde{\operatorname{ch}}\left(\mathcal{E}, H(\mathcal{E}), h^{\mathcal{E}}, h^{H(\mathcal{E})}\right) \\
& \quad=\sum_{k=0}^{\infty} \widetilde{\operatorname{ch}}\left(\mathcal{E}_{k}, \mathcal{E}_{k+1}, h^{\mathcal{E}_{k}}, h^{\mathcal{E}_{k+1}}\right)-\widetilde{\operatorname{ch}}\left(H(\mathcal{E}), \mathcal{E}_{\infty}, h^{H(\mathcal{E})}, h^{\mathcal{E}_{\infty}}\right)
\end{aligned}
$$

Preuve. - On le démontrera dans [Ma1].

b) Suite spectrale de Leray [Grot].

Soit $Y$ un espace topologique. On note $C^{Y}$ la catégorie des faisceaux de groupes abéliens sur $Y$. Pour un objet $G$ de $C^{Y}$, on note $\Gamma_{Y}(G)$ le groupe des sections de $G$ sur $Y$.

DÉfinition 10.8. - Soit $G$ un faisceau de groupes abéliens, et soit $L=\left(L^{i}\right)$ un complexe à degrés positifs de faisceaux de groupes abéliens. Soit $\varepsilon: G \rightarrow L$ une application d'augmentation. On dit que le complexe $L$ est une résolution de $G$, si la suite

$$
0 \rightarrow G \stackrel{\varepsilon}{\longrightarrow} L^{0} \rightarrow L^{1} \cdots \rightarrow L^{i} \rightarrow \cdots
$$

est exacte. On dit que le complexe $L$ est une résolution injective (resp. acyclique) de $G$, si de plus les $L^{i}$ sont injectifs (resp. acycliques).

Si $(F, v)$ est un complexe dans $C^{Y}$, on note, pour $i \in \mathbb{Z}$,

$$
\begin{gathered}
Z^{i}(F)=\operatorname{Ker}\left(v_{\mid F^{i}}\right), \quad B^{i}(F)=\operatorname{Im}\left(v_{\mid F^{i-1}}\right), \\
H^{i}(F)=Z^{i}(F) / B^{i}(F) .
\end{gathered}
$$

Soit $L=\left(L^{p, q}\right)$ un bicomplexe dans $C^{Y}$; on définit aussi $Z^{p, q}(L), B^{p, q}(L)$, $H^{p, q}(L)$ comme (10.23) pour le complexe $L^{\bullet, q}=\left(L^{p, q}\right)_{p}$. 
DÉfinition 10.9. - On dit que le bicomplexe $L=\left(L^{p, q}\right)_{p>0}$ est une résolution du complexe $F=\left(F^{p}\right)_{p \geq 0}$ s'il existe une augmentation $F \rightarrow L$, telle que pour $p$ fixé, $L^{p, \bullet}$ est une résolution de $F^{p}$, et si on filtre $L$ par $F^{q} L=\bigoplus_{q^{\prime} \geq q} L^{\bullet, q^{\prime}}$, alors la suite spectrale associée $\left(\mathcal{E}_{r}(L), d_{r}\right)$ dégénère à partir de $\mathcal{E}_{2}(L)$. On dit que le bicomplexe $L=\left(L^{p, q}\right)$ est une résolution injective (resp. acyclique) de $F$, si de plus les $L^{p, q}, H^{p, q}(L)$ sont injectifs (resp. acycliques).

Remarque 10.10. - Le bicomplexe $L=\left(L^{p, q}\right)$ est une résolution du complexe $F$ si et seulement si, pour $p$ fixé, $L^{p, \bullet}\left(\operatorname{resp} . Z^{p, \bullet}(L), \operatorname{resp} . B^{p, \bullet}(L)\right.$, resp. $\left.H^{p, \bullet}(L)\right)$ est une résolution de $F^{p}\left(\operatorname{resp} . Z^{p}(F)\right.$, resp. $B^{p}(F)$, resp. $\left.H^{p}(F)\right)$ [CE, chap. XVII].

De même le bicomplexe $L=\left(L^{p, q}\right)$ est une résolution injective (resp. acyclique) de $F$, si et seulement si de plus les $L^{p, q}, Z^{p, q}(L), B^{p, q}(L), H^{p, q}(L)$ sont injectifs (resp. acycliques).

Soient $X, Y$ deux espaces topologiques. Soit $f$ une application continue de $Y$ dans $X$. Soit $G$ un objet de $C^{Y}$, soit $\left(G^{p}\right)$ une résolution de $G$ dans $C^{Y}$. Soit $L=\left(L^{p, q}\right)$ un bicomplexe qui est une résolution injective du complexe $F=\left(f_{*}\left(G^{p}\right)\right)$ dans $C^{X}$. Alors on obtient un bicomplexe $\Gamma_{X}(L)$. Si on le filtre par $F^{p} \Gamma_{X}(L)=\bigoplus_{p^{\prime} \geq p} \Gamma_{X}\left(L^{\bullet}, p^{\prime}\right)$, on obtient une suite spectrale associée $\left(E_{r}, d_{r}\right)$ dont le terme $E_{2}$ est

$$
E_{2}^{p, q}(G)=H^{p}\left(X, R^{q} f_{*}(G)\right)
$$

et dont le terme $E_{\infty}$ est le groupe gradué associé à une filtration convenable de $\left(H^{n}(Y, G)\right)_{n}$.

Le résultat suivant est établi dans [Grot, th. 3.7.3].

ThÉORÈme 10.11. - Soient $X, Y$ deux espaces topologiques. Soit $f$ une application continue de $Y$ dans $X$. Alors il existe un foncteur spectral sur la catégorie $C^{Y}$ des faisceaux de groupes abéliens $G$ sur $Y$, aboutissant au foncteur gradué $\left(H^{n}(Y, G)\right)_{n}$, et dont le terme initial est

$$
E_{2}^{p, q}(G)=H^{p}\left(X, R^{q} f_{*}(G)\right) .
$$

De plus, ce foncteur spectral est calculé de la manière ci-dessus.

Remarque 10.12. - Pour calculer la suite spectrale de Leray, d'après [Grot, p. 147 et 149, rem. 2], on sait qu'on peut prendre aussi une résolution $\mathcal{G}$ de $G$ par des $G^{i}$ qui sont $f_{*}$-acycliques (i.e. $R^{j} f_{*} G^{i}=0$, pour $j>0$ et $i \geq 0$ ), et qu'il suffit de supposer que $L=\left(L^{q, p}\right)$ est une résolution acyclique du complexe $\left(f_{*} G^{q}\right)$.

\section{c) Un bicomplexe holomorphe sur $S$.}

Dans cette partie, en procédant comme dans la partie b), on construit la suite spectrale de Leray dans le contexte holomorphe.

TOME $127-1999-\mathrm{N}^{\circ} 4$ 
Définition 10.13. - Soit $(F, v)=\left(F^{i}\right)$ un complexe $\left(\right.$ resp. $\mathcal{F}=\left(F^{i, j}\right)$ un bicomplexe) de fibrés vectoriels holomorphes sur une variété complexe $V$. On suppose que les $H^{i}(F)$ sont des fibrés vectoriels holomorphes. On dit que le bicomplexe $\mathcal{F}$ est une résolution de fibrés holomorphes du complexe $F$, si les $H^{i, j}$ sont des fibrés vectoriels holomorphes et le bicomplexe $\mathcal{F}$ vérifie les conditions de la définition 10.9 dans le contexte holomorphe. Si de plus, les $F^{i, j}$ et les $H^{i, j}$ sont des fibrés acycliques, on dit que le bicomplexe $\mathcal{F}$ est une résolution acyclique de fibrés holomorphes du complexe $F$.

Remarque 10.14. - Désormais, si $\mathcal{F}=\left(F^{i, j}\right)$ est une résolution acyclique de fibrés holomorphes du complexe $F$ sur $V$, alors on note $\left(\mathcal{E}_{r}(\mathcal{F}), d_{r}\right)$ (resp. $\left.\left(E_{r}(\mathcal{F}), d_{r}\right)\right)$ la suite spectrale associée au bicomplexe $\mathcal{F}$ filtré par $F^{p} \mathcal{F}=$ $\bigoplus_{p^{\prime} \geq p} F^{\bullet, p}$ (resp. $H^{0}(V, \mathcal{F})$ filtré par $\left.F^{p} H^{0}(V, \mathcal{F})=\bigoplus_{p^{\prime} \geq p} H^{0}\left(V, F^{\bullet, p^{\prime}}\right)\right)$. D'après la définition 10.13 , on a

$$
E_{1}(\mathcal{F})=H^{0}\left(V, \mathcal{E}_{1}(\mathcal{F})\right), \quad E_{2}^{p, q}(\mathcal{F})=H^{p}\left(V, H^{q}(F)\right) .
$$

On rappelle que $V$ et $W$ sont des variétés projectives. Soit $n=\operatorname{dim} Z$.

En procédant comme dans [GrH, $\S 5.3]$ et [Q1, $\S 7.2 .7]$, on peut construire une résolution $\pi_{1 *}$ et $\pi_{3 *}$ acyclique $\left(\xi^{i}\right)_{0 \leq i \leq n}$ de fibrés holomorphes de $\xi$ sur $W$. On note $F^{i}=R^{0} \pi_{1 *} \xi^{i}(i \geq 0)$. Alors $F^{i}$ est $\pi_{2 *}$-acyclique. On a aussi un complexe $F$ de fibrés holomorphes sur $V$

$$
(F, v): \quad 0 \rightarrow F^{0} \stackrel{v}{\longrightarrow} F^{1} \stackrel{v}{\longrightarrow} \cdots \stackrel{v}{\longrightarrow} F^{n} \rightarrow 0 .
$$

Alors d'après la construction de l'image directe, on sait que pour $i \geq 0$,

$$
H^{i}(F)=R^{i} \pi_{1 *} \xi
$$

Comme le rang de $H^{i}(F)$ est localement constant, on sait que les rangs de $Z^{i}(F), B^{i}(F)$ sont aussi localement constants. Donc $Z^{i}(F), B^{i}(F)$ sont des fibrés vectoriels holomorphes sur $V$.

D'après [BS, preuve du lemme 12], on sait qu'on peut construire des fibrés vectoriels holomorphes $B^{i, j}, Z^{i, j}, H^{i, j}, F^{i, j}$ (avec $0 \leq i, j \leq n$ ) sur $V$ tels que pour chaque fibre $Y_{s}(s \in S)$, pour tout $i \geq 0$, le bicomplexe

$$
\begin{gathered}
0 \rightarrow Z^{i, \bullet} \longrightarrow F^{i, \bullet} \longrightarrow B^{i+1, \bullet} \rightarrow 0, \\
\left(\text { resp. } 0 \rightarrow B^{i, \bullet} \longrightarrow Z^{i, \bullet} \longrightarrow H^{i, \bullet} \rightarrow 0\right)
\end{gathered}
$$

est une résolution $\pi_{2 *}$-acyclique de fibrés holomorphes du complexe

$$
\begin{gathered}
0 \rightarrow Z^{i}(F) \longrightarrow F^{i} \longrightarrow B^{i+1}(F) \rightarrow 0, \\
\left(\text { resp. } 0 \rightarrow B^{i}(F) \longrightarrow Z^{i}(F) \longrightarrow H^{i}(F) \rightarrow 0\right)
\end{gathered}
$$

au sens de la définition 10.13 . 
Maintenant, en composant les bicomplexes

$$
0 \rightarrow Z^{i, \bullet} \longrightarrow F^{i, \bullet} \longrightarrow B^{i+1, \bullet} \rightarrow 0
$$

et les injections $B^{i, \bullet} \rightarrow Z^{i, \bullet}$, on obtient un bicomplexe $\left(F^{i, j}\right)$ de fibrés holomorphes sur $V$ qui, pour chaque fibre $Y_{s}(s \in S)$, est une résolution $\pi_{2 *}$-acyclique de fibrés holomorphes du complexe $\left(F^{i}\right)$ au sens de la définition 10.13.

Désormais, on note $\left(\xi^{i}\right)_{0 \leq i \leq n}$ une résolution $\pi_{1 *}$ et $\pi_{3 *}$ acyclique de fibrés holomorphes de $\xi$ sur $W$, et on note $F^{i}=R^{0} \pi_{1 *} \xi^{i}$. Soit $\mathcal{F}=\left(\mathcal{F}^{i, j}\right)$ une résolution $\pi_{2 *}$-acyclique de fibrés holomorphes du complexe $\left(F^{i}\right)$ au sens de la définition 10.13. On note :

$$
v: \mathcal{F}^{i, j} \rightarrow \mathcal{F}^{i+1, j}, \quad \delta: \mathcal{F}^{i, j} \rightarrow \mathcal{F}^{i, j+1},
$$

les morphismes du bicomplexe $\mathcal{F}$ et $d=v+\delta$ le morphisme total.

D'après la partie b), si on filtre le bicomplexe de fibrés holomorphes $E(\mathcal{F})=$ $\left(H^{0}(Y, \mathcal{F}), v, \delta\right)$ sur $S$ par ${ }^{\prime \prime} F^{p} E(\mathcal{F})=\bigoplus_{p^{\prime} \geq p} H^{0}\left(Y, \mathcal{F}^{\bullet}, p^{\prime}{ }_{\mid Y}\right)$, alors la suite spectrale associée $\left(E_{r}(\mathcal{F}), d_{r}\right)$ calcule la suite spectrale de Leray $\left(E_{r}, d_{r}\right)$ (à partir de $r=2$ ) pour $s \in S$.

d) Définition de $\widetilde{\operatorname{ch}}\left(E_{2}, H\left(Z, \xi_{\mid Z}\right), h^{E_{2}}, h^{H(Z, \xi \mid Z)}\right)$.

On se donne des métriques hermitiennes $h^{E^{p, q}(\mathcal{F})}$ et $h^{E_{1}^{p, q}(\mathcal{F})}$ sur $E^{p, q}(\mathcal{F})=$ $H^{0}\left(Y, \mathcal{F}^{p, q}{ }_{\mid Y}\right)$ et $E_{1}^{p, q}(\mathcal{F})$. Soit $h^{E_{2}(\mathcal{F})}$ la métrique sur $E_{2}(\mathcal{F})$ correspondant à $h^{E_{2}}$.

D'après la partie c) et le théorème 10.7, on a naturellement :

Définition 10.15. - On définit la classe de Bott-Chern

$$
\widetilde{\mathrm{ch}}\left(E_{2}, H\left(Z, \xi_{\mid Z}\right), h^{E_{2}}, h^{H(Z, \xi \mid Z)}\right) \in P^{S} / P^{S, 0}
$$

de la manière suivante:

$$
\begin{aligned}
\widetilde{\operatorname{ch}}\left(E_{2}, H\left(Z, \xi_{\mid Z}\right), h^{E_{2}}, h^{H(Z, \xi \mid Z)}\right) & \widetilde{\operatorname{ch}}\left(E(\mathcal{F}), H(E(\mathcal{F})), h^{E(\mathcal{F})}, h^{H(Z, \xi \mid Z)}\right) \\
& -\sum_{i=0}^{1} \widetilde{\operatorname{ch}}\left(E_{i}(\mathcal{F}), E_{i+1}(\mathcal{F}), h^{E_{i}(\mathcal{F})}, h^{E_{i+1}(\mathcal{F})}\right) .
\end{aligned}
$$

Remarque 10.16. - En utilisant [BGS1, th. 1.20] et la proposition 10.2, on sait que la définition de $\widetilde{c h}(\cdot, \cdot)$ ne dépend pas des choix de métriques $h^{E(\mathcal{F})}$ sur $E(\mathcal{F})$. De plus, on a

$$
\begin{aligned}
\frac{\bar{\partial} \partial}{2 i \pi} \tilde{\operatorname{ch}}\left(E_{2}, H\left(Z, \xi_{\mid Z}\right), h^{E_{2}}, h^{H(Z, \xi \mid Z)}\right) \\
=\operatorname{ch}\left(H\left(Z, \xi_{\mid Z}\right), h^{H(Z, \xi \mid Z)}\right)-\operatorname{ch}\left(E_{2}, h^{E_{2}}\right) .
\end{aligned}
$$

TOME $127-1999-\mathrm{N}^{\circ} 4$ 
ThÉORÈme 10.17. - La classe de Bott-Chern

$$
\widetilde{\mathrm{ch}}\left(E_{2}, H\left(Z, \xi_{\mid Z}\right), h^{E_{2}}, h^{H(Z, \xi \mid Z)}\right) \in P^{S} / P^{S, 0}
$$

ne dépend pas du choix des résolutions $\left(\xi^{\bullet}\right)$ et $\mathcal{F}$.

Preuve.

- Par [BS, $\S 4$ ], étant donné $\left(\xi_{1}^{\bullet}\right),\left(\xi_{2}^{\bullet}\right)$ deux résolutions $\pi_{1 *}$ et $\pi_{3 *}$-acycliques de $\xi$, on peut trouver une troisième résolution $\left(\xi_{3}^{\bullet}\right) \pi_{1 *}$ et $\pi_{3 *}$-acyclique et des injections $\mu_{1}, \mu_{2}$ des deux premières dans la troisième.

- Soient $F_{i}=R^{0} \pi_{1 *} \xi_{i}^{\bullet}$ où $i=1,2,3$. Par [BS, §4], étant données $\mathcal{F}_{i}$ une résolution $\pi_{2 *}$-acyclique de fibrés holomorphes du complexe $F_{i}(i=1,2)$, on peut trouver $\mathcal{F}_{3,1}, \mathcal{F}_{3,2}$ des résolutions $\pi_{2 *}$-acycliques de fibrés holomorphes du complexe $F_{3}$, et des injections $\mu_{i}: \mathcal{F}_{i} \rightarrow F_{3, i}(i=1,2)$ compatible à $\mu_{i}: F_{i} \rightarrow F_{3}$, et qui induisent des injections de $\mathcal{E}_{1}\left(\mathcal{F}_{i}\right)$ dans $\mathcal{E}_{1}\left(\mathcal{F}_{3, i}\right)$. Pour appliquer [BS, § 4], il suffit de considérer au cas où $F_{i}(i=1,2,3)$ sont des suites exactes courtes.

- Enfin, par [BS, §4], on peut trouver une troisième résolution $\mathcal{F}_{3}, \pi_{2 *}$ acyclique de fibrés holomorphes du complexe $F_{3}$ et des applications injectives des deux premières dans la troisième qui induisent des injections de $\mathcal{E}_{1}\left(\mathcal{F}_{3,1}\right), \mathcal{E}_{1}\left(\mathcal{F}_{3,2}\right)$ dans $\mathcal{E}_{1}\left(\mathcal{F}_{3}\right)$.

D'après les discussions ci-dessus, pour montrer le théorème, il suffit de montrer que, dans la situation suivante, les termes à droite associés à ces résolutions sont égaux.

Soit $\left(\xi^{i}\right),\left({ }^{\prime} \xi^{i}\right)$ deux résolutions $\pi_{1 *}$ et $\pi_{3 *}$ acycliques de fibrés holomorphes de $\xi$ sur $W$, et soit $\mu: \xi^{\bullet} \rightarrow^{\prime} \xi^{\bullet}$ une injection de complexe. Soit $F^{i}=R^{0} \pi_{1 *} \xi^{i}$, $G^{i}=R^{0} \pi_{1 *}{ }^{\prime} \xi^{i}$, alors on a une injection de complexe $\mu: F \rightarrow G$. Soit $\mathcal{F}=\left(\mathcal{F}^{i, j}\right)$ (resp. $\left.\mathcal{G}=\left(\mathcal{G}^{i, j}\right)\right)$ une résolution $\pi_{2 *}$-acyclique de fibrés holomorphes du complexe $F=\left(F^{i}\right)$ (resp. $G=\left(G^{i}\right)$ ) sur $V$. Soit $\mu: \mathcal{F} \rightarrow \mathcal{G}$ une injection de bicomplexes qui est compatible aux augmentations $F \rightarrow \mathcal{F}$ et $G \rightarrow \mathcal{G}$, et qui induit également une injection $\mu: \mathcal{E}_{1}(\mathcal{F}) \rightarrow \mathcal{E}_{1}(\mathcal{G})$.

Alors, on a le diagramme commutatif suivant :

$$
\begin{aligned}
& 0 \rightarrow \mathcal{F} \stackrel{\mu}{\longrightarrow} \mathcal{G} \longrightarrow \mathcal{G} / \mathcal{F} \rightarrow 0 \\
& 0 \rightarrow F \stackrel{\mu}{\longrightarrow} G \longrightarrow G / F \rightarrow 0 .
\end{aligned}
$$

Par un argument de suite spectrale, le complexe $G / F$ est acyclique.

Par (10.31), on a aussi le bicomplexe

$$
\prod_{\ldots \delta}^{\uparrow \mathcal{E}_{1}^{\bullet, q}(\mathcal{F}) \rightarrow \mathcal{E}_{1}^{\bullet, q}(\mathcal{G}) \rightarrow \mathcal{E}_{1}^{\bullet, q}(\mathcal{G} / \mathcal{F}) \rightarrow \mathcal{E}_{1}^{\bullet, q+1}(\mathcal{F}) \rightarrow \cdots}
$$

dont chaque ligne est acyclique. 
Par la définition 10.13 et (10.32), et par un argument de suite spectrale, on a

$$
\mathcal{E}_{2}(\mathcal{G} / \mathcal{F})=0 \text {. }
$$

Comme $\mu: \mathcal{E}_{1}(\mathcal{F}) \rightarrow \mathcal{E}_{1}(\mathcal{G})$ est injective, par (10.32), on a la suite exacte

$$
0 \rightarrow \mathcal{E}_{1}(\mathcal{F}) \longrightarrow \mathcal{E}_{1}(\mathcal{G}) \longrightarrow \mathcal{E}_{1}(\mathcal{G} / \mathcal{F}) \rightarrow 0
$$

Comme $\mathcal{F}, \mathcal{G}, \mathcal{E}_{1}(\mathcal{F})$ et $\mathcal{E}_{1}(\mathcal{G})$ sont $\pi_{2 *}$-acycliques, par $(10.31)$ et $(10.34), \mathcal{G} / \mathcal{F}$ et $\mathcal{E}_{1}(\mathcal{G} / \mathcal{F})$ sont aussi $\pi_{2 *}$-acycliques. D'où $\mathcal{G} / \mathcal{F}$ est une résolution $\pi_{2 *}$-acyclique de fibrés holomorphes du complexe $G / F$.

D'après la remarque 10.14 , on sait que

$$
E_{1}(\mathcal{G} / \mathcal{F})=H^{0}\left(Y, \mathcal{E}_{1}(\mathcal{G} / \mathcal{F})_{\mid Y}\right), \quad E_{2}(\mathcal{G} / \mathcal{F})=0 .
$$

Comme $\mathcal{E}_{1}(\mathcal{F}), \mathcal{E}_{1}(\mathcal{G})$ sont $\pi_{2 *}$-acycliques, par $(10.25),(10.34)$ et $(10.35)$, on a la suite exacte suivante :

$$
E_{1}(\mathcal{G}, \mathcal{F}): 0 \rightarrow E_{1}(\mathcal{F}) \longrightarrow E_{1}(\mathcal{G}) \longrightarrow E_{1}(\mathcal{G} / \mathcal{F}) \rightarrow 0
$$

Si on note $I(\mathcal{F}), I(\mathcal{G})$ les termes à droite de $(10.29)$ associés à $\mathcal{F}, \mathcal{G}$, alors on doit montrer que

$$
I(\mathcal{F})=I(\mathcal{G}) \text { dans } P^{S} / P^{S, 0}
$$

Dans la suite, pour simplifier les notations, on note $E(\mathcal{G}, \mathcal{F})$ la suite exacte suivante :

$$
E(\mathcal{G}, \mathcal{F}): 0 \rightarrow E(\mathcal{F}) \longrightarrow E(\mathcal{G}) \longrightarrow E(\mathcal{G} / \mathcal{F}) \rightarrow 0
$$

Soient $h^{E_{r}(\mathcal{F})}, h^{E_{r}(\mathcal{G})}, h^{E_{r}(\mathcal{G} / \mathcal{F})}$ (avec $r=0,1,2$ ) des métriques hermitiennes sur $E_{r}(\mathcal{F}), E_{r}(\mathcal{G}), E_{r}(\mathcal{G} / \mathcal{F})$; soient $h^{E(\mathcal{G}, \mathcal{F})}, h^{E_{1}(\mathcal{G}, \mathcal{F})}$ les métriques induites sur $E(\mathcal{G}, \mathcal{F}), E_{1}(\mathcal{G}, \mathcal{F})$.

Pour le bicomplexe

$$
\begin{array}{ccc}
\uparrow_{d} & \uparrow_{d} & \uparrow_{d} \\
0 \rightarrow E(\mathcal{F}) & \longrightarrow E(\mathcal{G}) \longrightarrow E(\mathcal{G} / \mathcal{F}) \rightarrow 0,
\end{array}
$$

en utilisant le théorème 10.7 , on a

$$
\begin{gathered}
\tilde{\operatorname{ch}}\left(E(\mathcal{F}), H(E(\mathcal{F})), h^{E(\mathcal{F})}, h^{H(Z, \xi \mid Z)}\right) \\
-\widetilde{\operatorname{ch}}\left(E(\mathcal{G}), H(E(\mathcal{G})), h^{E(\mathcal{G})}, h^{H(Z, \xi \mid Z)}\right) \\
+\widetilde{\operatorname{ch}}\left(E(\mathcal{G} / \mathcal{F}), h^{E(\mathcal{G} / \mathcal{F})}\right) \\
\quad=\widetilde{\operatorname{ch}}\left(E(\mathcal{G}, \mathcal{F}), h^{E(\mathcal{G}, \mathcal{F})}\right) \text { dans } P^{S} / P^{S, 0} .
\end{gathered}
$$

TOME $127-1999-\mathrm{N}^{\circ} 4$ 
Pour le bicomplexe

$$
\begin{array}{ccc}
\uparrow v & \uparrow v & \uparrow v \\
0 \rightarrow E(\mathcal{F}) & \longrightarrow E(\mathcal{G}) & \longrightarrow E(\mathcal{G} / \mathcal{F}) \rightarrow 0,
\end{array}
$$

en utilisant le théorème 10.7 et (10.36), on a

$$
\begin{aligned}
& \widetilde{\operatorname{ch}}\left(E(\mathcal{F}), E_{1}(\mathcal{F}), h^{E(\mathcal{F})}, h^{E_{1}(\mathcal{F})}\right) \\
& \text { - } \widetilde{\operatorname{ch}}\left(E(\mathcal{G}), E_{1}(\mathcal{G}), h^{E(\mathcal{G})}, h^{E_{1}(\mathcal{G})}\right) \\
& +\widetilde{\operatorname{ch}}\left(E(\mathcal{G} / \mathcal{F}), E_{1}(\mathcal{G} / \mathcal{F}), h^{E(\mathcal{G} / \mathcal{F})}, h^{E_{1}(\mathcal{G} / \mathcal{F})}\right) \\
& +\widetilde{\operatorname{ch}}\left(E_{1}(\mathcal{G}, \mathcal{F}), h^{E_{1}(\mathcal{G}, \mathcal{F})}\right) \\
& =\widetilde{\operatorname{ch}}\left(E(\mathcal{G}, \mathcal{F}), h^{E(\mathcal{G}, \mathcal{F})}\right) \text { dans } P^{S} / P^{S, 0} .
\end{aligned}
$$

Pour le bicomplexe

$$
\begin{array}{ccc}
\uparrow \delta & \uparrow \delta & \uparrow \delta \\
0 \rightarrow E_{1}(\mathcal{F}) & \longrightarrow E_{1}(\mathcal{G}) & \longrightarrow E_{1}(\mathcal{G} / \mathcal{F}) \rightarrow 0
\end{array}
$$

en utilisant le théorème 10.7 et (10.36), on a

$$
\begin{aligned}
& \tilde{\operatorname{ch}}\left(E_{1}(\mathcal{F}), E_{2}(\mathcal{F}), h^{E_{1}(\mathcal{F})}, h^{E_{2}}\right) \\
& -\widetilde{c h}\left(E_{1}(\mathcal{G}), E_{2}(\mathcal{G}), h^{E_{1}(\mathcal{G})}, h^{E_{2}}\right) \\
& +\widetilde{c h}\left(E_{1}(\mathcal{G} / \mathcal{F}), h^{E_{1}(\mathcal{G} / \mathcal{F})}\right) \\
& \quad=\widetilde{c h}\left(E_{1}(\mathcal{G}, \mathcal{F}), h^{E_{1}(\mathcal{G}, \mathcal{F})}\right) \quad \text { dans } \quad P^{S} / P^{S, 0} .
\end{aligned}
$$

Pour le bicomplexe $E(\mathcal{G} / \mathcal{F})$, en utilisant le théorème 10.7 et $(10.35)$, on a

$$
\begin{aligned}
& \widetilde{\operatorname{ch}}\left(E(\mathcal{G} / \mathcal{F}), E_{1}(\mathcal{G} / \mathcal{F}), h^{E(\mathcal{G} / \mathcal{F})}, h^{E_{1}(\mathcal{G} / \mathcal{F})}\right) \\
& +\widetilde{\operatorname{ch}}\left(E_{1}(\mathcal{G} / \mathcal{F}), h^{E_{1}(\mathcal{G} / \mathcal{F})}\right) \\
& \quad=\widetilde{\operatorname{ch}}\left(E(\mathcal{G} / \mathcal{F}), h^{E(\mathcal{G} / \mathcal{F})}\right) \text { dans } \quad P^{S} / P^{S, 0} .
\end{aligned}
$$

Par (10.40)-(10.45), on obtient (10.37).

On a bien terminé la preuve du théorème 10.17 .

\section{e) Preuve du théorème 3.9.}

Par la construction de la section canonique $\sigma \in \lambda^{-1}\left(E_{2}\right) \otimes \lambda\left(R^{\bullet} \pi_{3 *} \xi\right)[\mathrm{KM}]$, en utilisant [BGS3, th. 1.23], (10.7), (10.29), on obtient le théorème 3.9. On laisse le détail au lecteur. 


\section{Identités entre les formes de torsion analytique d'un complexe de fibrés holomorphes}

Soit $\pi: M \rightarrow B$ une submersion holomorphe de fibre compacte $Z$. Soit $\left(\eta^{i}, v\right)_{0 \leq i \leq n}$ un complexe holomorphe de fibrés vectoriels holomorphes hermitiens sur $M$. On suppose que le rang de l'hypercohomologie $H\left(Z, \eta_{\mid Z}\right)$ est localement constant sur $B$. Dans ce paragraphe, on montre des relations entre les formes de torsion analytique du complexe $(\eta, v)$.

Ce paragraphe est organisé de la façon suivante. Dans a), on rappelle la définition de formes de torsion analytique pour le complexe $(\eta, v)$ (voir [B4, $\S 3(\mathrm{~b})]$ ). Dans b), on démontre une identité de formes de torsion analytique associées à $(\eta, v)$. Dans $\mathrm{c})$, on énonce une identité entre les formes de torsion analytique de $(\eta, v)$ et celles de son fibré cohomologique. Dans d), en déformant le complexe $\eta$ (voir [BGS1]), on montre le théorème 11.2.

On utilise les notations du paragraphe 2.

\section{a) Formes de torsion analytique associées à un complexe.}

Soient $M$ et $B$ des variétés complexes. Soit $\pi: M \rightarrow B$ une submersion holomorphe de fibre compacte $Z$. Soit $\omega^{M}$ une $(1,1)$-forme réelle, fermée, $\mathcal{C}^{\infty}$ sur $M$ qui induit une métrique hermitienne $g^{T Z}$ sur $T Z$. Soit

$$
(\eta, v): 0 \rightarrow \eta^{0} \stackrel{v}{\longrightarrow} \eta^{1} \stackrel{v}{\longrightarrow} \cdots \stackrel{v}{\longrightarrow} \eta^{n} \rightarrow 0
$$

un complexe holomorphe de fibrés vectoriels holomorphes sur $M$. Soient $h^{\eta^{i}}$ une métrique hermitienne sur $\eta^{i}$, et $h^{\eta}=\bigoplus_{i} h^{\eta^{i}}$ la métrique correspondante sur $\eta=\bigoplus_{i=0}^{n} \eta^{i}$.

Pour $b \in B$, soit $E_{b}$ l'espace des sections $\mathcal{C}^{\infty}$ de $\Lambda\left(T^{*(0,1)} Z\right) \otimes \eta \operatorname{sur} Z_{b}$. Soient $N_{Z}$ et $N_{H}$ les opérateurs de nombre sur $\Lambda\left(T^{*(0,1)} Z\right)$ et $\eta$. Alors l'opérateur $N_{Z}+N_{H}$ définit une $\mathbb{Z}$-graduation sur $E$. Soit $\bar{\partial}^{Z}$ l'opérateur de Dolbeault agissant sur $E$. Alors $\left(E, \bar{\partial}^{Z}+v\right)$ est un complexe $\mathbb{Z}$-gradué. Soit $H\left(Z_{b}, \eta_{\mid Z_{b}}\right)$ l'hypercohomologie de $\left(\mathcal{O}_{Z}\left(\eta_{\mid Z}\right), v\right)$ sur $Z$. Alors

$$
H\left(Z_{b}, \eta_{\mid Z_{b}}\right) \simeq H\left(E_{b}, \bar{\partial}^{Z}+v\right)
$$

On suppose que le rang des fibres $H\left(E, \bar{\partial}^{Z}+v\right)$ est localement constant. Alors $H\left(E, \bar{\partial}^{Z}+v\right)$ est un fibré holomorphe sur $B$. Dans la suite, on identifie les fibrés holomorphes $H\left(E, \bar{\partial}^{Z}+v\right)$ et $H\left(Z, \eta_{\mid Z}\right)$.

Soit $\bar{\partial}^{Z *}$ (resp. $v^{*}$ ) l'adjoint de $\bar{\partial}^{Z}$ (resp. $v$ ) relativement à $g^{T Z}, h^{\eta}$ (resp. $h^{\eta}$ ). On pose

$$
V=v+v^{*}, \quad D^{Z}=\bar{\partial}^{Z}+\bar{\partial}^{Z *}, \quad A^{Z}=D^{Z}+V .
$$

TOME $127-1999-\mathrm{N}^{\circ} 4$ 
Par la théorie de Hodge, pour $b \in B$,

$$
H\left(E_{b}, \bar{\partial}^{Z}+v\right) \simeq \operatorname{Ker} A^{Z_{b}} .
$$

Comme au paragraphe $2 \mathrm{~b}), \operatorname{Ker} A^{Z}$ hérite du produit (2.2) de $E$. Soit $h^{H(Z, \eta \mid Z)}$ la métrique hermitienne correspondante sur $H\left(Z, \eta_{\mid Z}\right)$. On pose

$$
\begin{aligned}
\operatorname{ch}\left(\eta, h^{\eta}\right) & =\sum_{i=0}^{n}(-1)^{i} \operatorname{ch}\left(\eta^{i}, h^{\eta^{i}}\right) \\
\operatorname{ch}\left(H\left(Z, \eta_{\mid Z}\right), h^{H(Z, \eta \mid Z)}\right) & =\sum_{i=0}^{n}(-1)^{i} \operatorname{ch}\left(H^{i}\left(Z, \eta_{\mid Z}\right), h^{H^{i}(Z, \eta \mid Z)}\right) .
\end{aligned}
$$

Par l'extension de [BKö] au complexe de fibrés hermitiens $\left(E, \bar{\partial}^{Z}+v\right)$, on peut associer des formes de torsion analytique ( $f f .[\mathrm{B} 4, \S 3 \mathrm{~b})]$ )

$$
T\left(\omega^{M}, \bar{\partial}^{Z}+v, h^{\eta}\right) \in P^{B} / P^{B, 0} .
$$

Pour cela, il suffit de remplacer partout $\bar{\partial}^{Z}$ par $\bar{\partial}^{Z}+v$. Les formes $T\left(\omega^{M}, \bar{\partial}^{Z}+\right.$ $\left.v, h^{\eta}\right)$ vérifient l'équation suivante :

$$
\begin{aligned}
\frac{\bar{\partial} \partial}{2 i \pi} T\left(\omega^{M}, \bar{\partial}^{Z}\right. & \left.+v, h^{\eta}\right) \\
& =\operatorname{ch}\left(H\left(Z, \eta_{\mid Z}\right), h^{H(Z, \eta \mid Z)}\right)-\int_{Z} \operatorname{Td}\left(T Z, g^{T Z}\right) \operatorname{ch}\left(\eta, h^{\eta}\right) .
\end{aligned}
$$

On va maintenant exprimer $T\left(\omega^{M}, \bar{\partial}^{Z}+v, h^{\eta}\right)$ en fonction des formes de torsion analytique associées aux deux filtrations du complexe $E$.

b) Formes de torsion analytique du complexe $E$ et filtration par $\bar{\partial}^{Z}$.

Dans cette partie, on suppose que $\eta$ est $\pi_{*}$-acylique, i.e. que l'on a $R^{j} \pi_{*} \eta^{i}=0$ pour tout $j>0$ et $i \geq 0$. Alors les $R^{0} \pi_{*} \eta^{i}$ sont des fibrés vectoriels holomorphes sur $B$.

On pose $\zeta^{i}=R^{0} \pi_{*} \eta^{i}$. Alors

$$
(\zeta, v): 0 \rightarrow \zeta^{0} \stackrel{v}{\longrightarrow} \zeta^{1} \stackrel{v}{\longrightarrow} \cdots \stackrel{v}{\longrightarrow} \zeta^{n} \rightarrow 0
$$

est aussi un complexe holomorphe de fibrés vectoriels holomorphes sur $B$. On note $H_{x}^{i}(\zeta)$ le $i$-ième groupe cohomologique du complexe $(\zeta, v)_{x}$.

Par un argument de suite spectrale, pour $i \geq 0, H^{i}(\zeta)$ et $H^{i}\left(E, \bar{\partial}^{Z}+v\right)$ sont isomorphes canoniquement et holomorphiquement. Dans la suite, on identifie les fibrés holomorphes $H(\zeta)$ et $H\left(Z, \eta_{\mid Z}\right)$ sur $B$. 
Soient $h^{\zeta}$ et $h^{H(\zeta)}$ les métriques hermitiennes sur $\zeta$ et $H(\zeta)$ induites par $\left(g^{T Z}, h^{\eta}\right)$, et $h^{\zeta}$. Soit $\tilde{\mathrm{ch}}\left(\zeta, H(\zeta), h^{\zeta}, h^{H(Z, \eta \mid Z)}\right) \in P^{B} / P^{B, 0}$ la classe de BottChern construite au paragraphe 10 a). Soient $T\left(\omega^{M}, h^{\eta^{i}}\right)$ les formes de torsion analytique sur $B$ associées à $\eta^{i}$. On pose

$$
T\left(\omega^{M}, h^{\eta}\right)=\sum_{i=0}^{n}(-1)^{i} T\left(\omega^{M}, h^{\eta^{i}}\right) .
$$

ThÉORÈme 11.1. - Dans $P^{B} / P^{B, 0}$, on a l'identité

$$
T\left(\omega^{M}, \bar{\partial}^{Z}+v, h^{\eta}\right)=T\left(\omega^{M}, h^{\eta}\right)+\widetilde{c h}\left(\zeta, H(\zeta), h^{\zeta}, h^{H(Z, \eta \mid Z)}\right) .
$$

Preuve. - La formule (11.9) est une généralisation de [B4, th. 0.2]. La seule différence est qu'on suppose $H^{i}(\zeta)=0$ pour $i<n$ dans [B4, $\left.\S 14\right]$. En utilisant la proposition 10.4, et en procédant comme en [B4, § 14], on a (11.9).

\section{c) Formes de torsion analytique du complexe $E$ et filtration par $v$.}

Dans cette partie, on suppose que $\eta$ vérifie l'une de deux conditions suivantes :

i) on a $H^{i}(\eta)=0$ pour $i>0$;

ii) le rang des fibres $H^{i}(\eta)$ est localement constant, et $H^{i}(\eta)$ est $\pi_{*}$-acyclique.

Par un argument de suite spectrale, on sait que $H\left(Z, H(\eta)_{\mid Z}\right)$ est un fibré vectoriel holomorphe sur $B$; de plus, $H\left(Z, H(\eta)_{\mid Z}\right)$ et $H\left(Z, \eta_{\mid Z}\right)$ sont isomorphes canoniquement et holomorphiquement. D'où dans les deux cas ci-dessus, on peut identifier les fibrés holomorphes $H\left(Z, H(\eta)_{\mid Z}\right)$ et $H\left(Z, \eta_{\mid Z}\right)$.

Soit $h^{H(\eta)}$ une métrique sur $H(\eta)$. Soit $h^{H(Z, H(\eta) \mid Z)}$ la métrique sur $H\left(Z, H(\eta)_{\mid Z}\right)$ induite par $g^{T Z}, h^{H(\eta)}$. Soit $\widetilde{\operatorname{ch}}\left(\eta, H(\eta), h^{\eta}, h^{H(\eta)}\right)$ la classe de Bott-Chern construite au paragraphe $10 \mathrm{a}$ ).

THÉORÈme 11.2. - On a l'identité suivante dans $P^{B} / P^{B, 0}$ :

$$
\begin{aligned}
T\left(\omega^{M}, \bar{\partial}^{Z}+v, h^{\eta}\right)=T\left(\omega^{M}, h^{H(\eta)}\right) & \\
-\widetilde{\operatorname{ch}}\left(H\left(Z, \eta_{\mid Z}\right),\right. & \left.h^{H(Z, \eta \mid Z)}, h^{H(Z, H(\eta) \mid Z)}\right) \\
& \quad+\int_{Z} \operatorname{Td}\left(T Z, g^{T Z}\right) \widetilde{\operatorname{ch}}\left(\eta, H(\eta), h^{\eta}, h^{H(\eta)}\right) .
\end{aligned}
$$

\section{d) Preuve du théorème 11.2.}

Soit $\mathbb{P}^{1}$ le plan projectif complexe. Soit $\mathcal{O}(1)$ le fibré en droite canonique de degré 1 sur $\mathbb{P}^{1}$, et soit $\sigma$ la section holomorphe de $\mathcal{O}(1)$ définie par $(1,0) \in \mathbb{C}^{2 *}$.

TOME $127-1999-\mathrm{N}^{\circ} 4$ 
Alors $\sigma$ n'est nulle qu'au point $\infty$. Si $F$ est un fibré vectoriel holomorphe sur $M \times \mathbb{P}^{1}$, et $m \geq 0$, on définit $F(m)$ de la manière suivante :

$$
F(0)=F, \quad F(m)=F(m-1) \otimes \mathcal{O}(1) .
$$

Pour $i \geq 0$, on pose $F^{i}=\operatorname{Im}\left(v_{\mid \eta^{i-1}}\right), G^{i}=\operatorname{ker} v_{\mid \eta^{i}}, H^{i}=H^{i}(\eta)$. Soit $h^{F^{i}}, h^{G^{i}}$ les métriques sur $F^{i}, G^{i}$ induites par $h^{\eta}$.

Pour $j=0, \ldots, n-1$, soit l'application $F^{j} \rightarrow G^{j} \oplus F^{j}(1)$ définie par l'inclusion dans $G^{j}$ et par Id $\otimes \sigma$ dans $F^{j}(1)$. Soit ${ }^{\prime} G^{j}=G^{j} \oplus F^{j}(1) / F^{j}$. Soit l'application $G^{j} \rightarrow \eta^{j} \oplus^{\prime} G^{j}(1)$ définie par l'inclusion dans $\eta^{j}$ et par $x \in G^{j} \mapsto[(x, 0)] \otimes \sigma \in{ }^{\prime} G^{j}(1)$. On définit aussi

$$
\left\{\begin{array}{l}
\tilde{\eta}^{j}=\operatorname{Coker}\left(G^{j} \rightarrow \eta^{j} \oplus^{\prime} G^{j}(1)\right)(2 n-2 j), \\
\widetilde{F}^{j}=F^{j}(2 n-2 j+2), \quad \widetilde{G}^{j}={ }^{\prime} G^{j}(2 n-2 j+1), \\
\widetilde{H}^{j}=H^{j}(2 n-2 j+1) .
\end{array}\right.
$$

Alors on a les suites exactes suivantes sur $M \times \mathbb{P}^{1}$

$$
\left\{\begin{array}{l}
0 \rightarrow \widetilde{G}^{j} \longrightarrow \tilde{\eta}^{j} \longrightarrow \widetilde{F}^{j+1} \rightarrow 0 \\
0 \rightarrow \widetilde{F}^{j+1} \longrightarrow \widetilde{G}^{j+1} \longrightarrow \widetilde{H}^{j+1} \rightarrow 0 .
\end{array}\right.
$$

En les composant, on obtient un complexe $(\tilde{\eta}, v)=\left(\tilde{\eta}^{i}, v\right)_{0 \leq i \leq n}$ sur $M \times \mathbb{P}^{1}$ (voir [GS4, p. 477]).

Soit $z$ le paramètre complexe standard sur $\mathbb{P}^{1}$, et soit $i_{z}: M \rightarrow M \times \mathbb{P}^{1}$ l'application de $x \in M$ à $(x, z) \in M \times \mathbb{P}^{1}$. Alors pour $z \neq \infty$, comme $\sigma(z) \neq 0$, le fibré $i_{z}^{*}(\tilde{\eta})$ est isomorphe à $\eta$, et $i_{\infty}^{*} \tilde{\eta}^{j} \simeq F^{j} \oplus F^{j+1} \oplus H^{j}$. Par partition de l'unité, on peut trouver une métrique $h^{\tilde{\eta}^{j}}$ sur $\tilde{\eta}^{j}$ telle que les isomorphismes $i_{0}^{*} \tilde{\eta}^{j} \simeq \eta^{j}$ et $i_{\infty}^{*} \tilde{\eta}^{j} \simeq F^{j} \oplus F^{j+1} \oplus H^{j}$ sont des isométries.

Proposition 11.3. - On a :

$$
\widetilde{\operatorname{ch}}\left(\eta, H(\eta), h^{\eta}, h^{H(\eta)}\right)=-\int_{\mathbb{P}^{1}} \operatorname{ch}\left(\tilde{\eta}, h^{\tilde{\eta}}\right) \log |z|^{2} .
$$

Preuve. - La preuve est la même que dans [BGS1, (1.115)].

Maintenant, on considère le diagramme commutatif suivant :

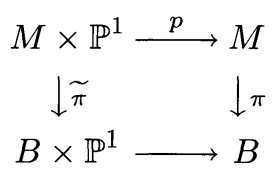

avec $\tilde{\pi}=\pi \times \operatorname{Id}_{\mathbb{P}^{1}}$. 
Soit $H\left(Z, \tilde{\eta}_{\mid Z}\right)$ l'hypercohomologie du complexe $\left(\mathcal{O}_{Z}\left(\tilde{\eta}_{\mid Z}\right), v\right)$ sur $Z$. Par notre hypothèse, le rang des fibres $H\left(Z, \tilde{\eta}_{\mid Z}\right)$ est localement constant. Donc les formes de torsion analytique

$$
T\left(p^{*} \omega^{M}, \bar{\partial}^{Z}+v, h^{\tilde{\eta}}\right) \in P^{B \times \mathbb{P}^{1}} / P^{B \times \mathbb{P}^{1}, 0}
$$

sont bien définies. On a aussi

$$
h^{i_{0}^{*} H(Z, \tilde{\eta} \mid Z)}=h^{H(Z, \eta \mid Z)}, \quad h_{\infty}^{i_{\infty}^{*} H(Z, \tilde{\eta} \mid Z)}=h^{H(Z, H(\eta) \mid Z)} .
$$

On a

$$
\frac{\bar{\partial} \partial}{2 i \pi} \log |z|^{2}=\delta_{0}-\delta_{\infty}
$$

En utilisant (2.18), (11.14) et (11.17), on obtient

$$
\begin{aligned}
\int_{Z} \operatorname{Td}(T Z, & \left.g^{T Z}\right) \tilde{\operatorname{ch}}\left(\eta, H(\eta), h^{\eta}, h^{H(\eta)}\right) \\
- & \tilde{\operatorname{ch}}\left(H\left(Z, \eta_{\mid Z}\right), h^{H(Z, \eta \mid Z)}, h^{H(Z, H(\eta) \mid Z)}\right) \\
& =\int_{\mathbb{P}^{1}} \frac{\bar{\partial} \partial}{2 i \pi} T\left(p^{*} \omega^{M}, \bar{\partial}^{Z}+v, h^{\tilde{\eta}}\right) \log |z|^{2} \\
& =i_{0}^{*} T\left(p^{*} \omega^{M}, \bar{\partial}^{Z}+v, h^{\tilde{\eta}}\right)-i_{\infty}^{*} T\left(p^{*} \omega^{M}, \bar{\partial}^{Z}+v, h^{\tilde{\eta}}\right) \\
& =T\left(\omega^{M}, \bar{\partial}^{Z}+v, h^{\eta}\right)-T\left(\omega^{M}, \bar{\partial}^{Z}+v, h_{\infty}^{i^{*} \tilde{\eta}}\right) .
\end{aligned}
$$

D'après notre construction de $\tilde{\eta}$, pour le complexe $i_{\infty}^{*} \tilde{\eta}$, on a :

$$
i_{\infty}^{*} \tilde{\eta}^{j}=F^{j} \oplus F^{j+1} \oplus H^{j}, \quad h^{i_{\infty}^{*} \tilde{\eta}^{j}}=h^{F^{j}} \oplus h^{F^{j+1}} \oplus h^{H^{j}}
$$

et l'application $v: i_{\infty}^{*} \tilde{\eta}^{j} \rightarrow i_{\infty}^{*} \tilde{\eta}^{j+1}$ est donnée par 0 sur $F^{j} \oplus H^{j}$ et Id sur $F^{j+1}$.

En utilisant (11.16), (11.19), on peut vérifier facilement qu'on a :

$$
T\left(\omega^{M}, \bar{\partial}^{Z}+v, h_{\infty}^{i_{\infty}^{*} \tilde{\eta}}\right)=T\left(\omega^{M}, h^{H(\eta)}\right) \quad \text { dans } \quad P^{B} / P^{B, 0}
$$

Par (11.18), (11.20), on a (11.10). 


\section{Preuve du théorème 3.11}

Nous allons appliquer les théorèmes 3.5, 11.1, 11.2 et le paragraphe 10.

On rappelle que $\left(\xi^{\bullet}, v\right)=\left(\xi^{i}, v\right)_{0 \leq i \leq n}$ est une résolution de fibrés holomorphes $\pi_{1 *}$ et $\pi_{3 *}$-acycliques de $\xi$ sur $W$. On pose $F^{i}=R^{0} \pi_{1 *} \xi^{i}$. Soit $\mathcal{F}=\left(\mathcal{F}^{i, j}\right)$ une résolution $\pi_{2 *}$-acyclique de fibrés holomorphes du complexe $F=\left(F^{i}\right)$ au sens de la définition 10.13. On note aussi $\left(\mathcal{E}_{r}(\mathcal{F}), d_{r}\right)\left(\operatorname{resp} .\left(E_{r}(\mathcal{F}), d_{r}\right)\right)$ la suite spectrale associée au bicomplexe $\mathcal{F}$ filtré par $F^{p} \mathcal{F}=\bigoplus_{p^{\prime} \geq p} \mathcal{F}^{\bullet}, p^{\prime}$ (resp. $H^{0}(Y, \mathcal{F})$ filtré par

$$
\left.F^{p} H^{0}(Y, \mathcal{F})=\bigoplus_{p^{\prime} \geq p} H^{0}\left(Y, \mathcal{F}^{\bullet}, p^{\prime}{ }_{\mid Y}\right)\right) .
$$

Soient $v: \mathcal{F}^{i, j} \rightarrow \mathcal{F}^{i+1, j}$ et $\delta: \mathcal{F}^{i, j} \rightarrow \mathcal{F}^{i, j+1}$ les différentielles du bicomplexe $\mathcal{F}$.

On rappelle que $h^{\xi}$ est une métrique hermitienne sur $\xi$, et que $h^{R \pi_{1 *} \xi}, h^{R \pi_{3 *} \xi}$ sont les métriques sur $R^{\bullet} \pi_{1 *} \xi, R^{\bullet} \pi_{3 *} \xi$ induites par $\left(g^{T X}, h^{\xi}\right),\left(g^{T Z}, h^{\xi}\right)$ définies au paragraphe $2 \mathrm{~b}$ ). Soit $h^{\xi^{\bullet}}=\bigoplus_{i} h^{\xi^{i}}$ une métrique hermitienne sur $\xi^{\bullet}$. Soit $h^{F}$ la métrique sur $F$ induite par $\left(g^{T Y}, h^{\xi^{\bullet}}\right)$.

Comme au paragraphe $11 \mathrm{a})$, on définit $T_{2}\left(\omega^{V}, \bar{\partial}^{Y}+v, h^{F}\right)\left(\operatorname{resp} . T_{3}\left(\omega^{W}, \bar{\partial}^{Z}+\right.\right.$ $\left.v, h^{\xi^{\bullet}}\right)$ ) les formes de torsion analytique sur $S$ associées à $\left(V, S, \pi_{2}\right)$ (resp. $\left.\left(W, S, \pi_{3}\right)\right)$.

Dans la suite, pour $\eta$ un fibré vectoriel holomorphe hermitien sur $V$, on note toujours $T_{2}\left(\omega^{V}, h^{\eta}\right)$ les formes de torsion analytique sur $S$ associées à $\left(V, S, \pi_{2}\right)$. Si $\left(L^{i}\right)_{0 \leq i \leq n}$ est un complexe de fibrés vectoriels holomorphes hermitiens sur $V$, on note

$$
T_{2}\left(\omega^{V}, h^{L}\right)=\sum_{i=0}^{n}(-1)^{i} T_{2}\left(\omega^{V}, h^{L^{i}}\right) .
$$

Soient $H\left(Z, \xi^{\bullet} \mid Z\right), \quad H\left(Y, F_{\mid Y}\right)$ les hypercohomologies de $\left(\mathcal{O}_{Z}\left(\xi^{\bullet} \mid Z\right), v\right)$, $\left(\mathcal{O}_{Y}\left(F_{\mid Y}\right), v\right)$. Soient $h^{H\left(Z, \xi^{\bullet} \mid Z\right)}, h^{H(Y, F \mid Y)}$ les métriques sur $H\left(Z, \xi^{\bullet} \mid Z\right)$, $H\left(Y, F_{\mid Y}\right)$ induites par $\left(g^{T Z}, h^{\xi^{\bullet}}\right),\left(g^{T Y}, h^{F}\right)$ comme au paragraphe 11 a). Comme $\xi^{\bullet}$ est une résolution $\pi_{1 *}$ et $\pi_{3 *}$-acyclique de $\xi$ sur $W$, on a :

$$
H\left(Z, \xi^{\bullet} \mid Z\right)=H\left(Y, F_{\mid Y}\right)=R^{\bullet} \pi_{3 *} \xi .
$$

En procédant comme aux paragraphes $4-9$, on a dans $P^{S} / P^{S, 0}$ :

$$
\begin{aligned}
T_{3}\left(\omega^{W}, \bar{\partial}^{Z}+v, h^{\xi^{\bullet}}\right)=\int_{Y} \operatorname{Td}\left(T Y, g^{T Y}\right) T_{1}\left(\omega^{W}, h^{\xi^{\bullet}}\right) \\
+T_{2}\left(\omega^{V}, \bar{\partial}^{Y}+v, h^{F}\right) \\
\quad-\int_{Z} \widetilde{\operatorname{Td}}\left(T Z, T Y, g^{T Z}, g^{T Y}\right) \operatorname{ch}\left(\xi^{\bullet}, h^{\xi^{\bullet}}\right) \\
+\widetilde{\operatorname{ch}}\left(R^{\bullet} \pi_{3 *} \xi, h^{H(Y, F \mid Y)}, h^{H\left(Z, \xi^{\bullet} \mid Z\right)}\right) .
\end{aligned}
$$


Pour le complexe $\xi^{\bullet}=\left(\xi^{i}\right)$, en utilisant les théorèmes 11.1 et 11.2 , on a :

$$
\begin{aligned}
T_{3}\left(\omega^{W}, h^{\xi}\right)=-\int_{Z} & \operatorname{Td}\left(T Z, g^{T Z}\right) \widetilde{\operatorname{ch}}\left(\xi^{\bullet}, \xi, h^{\xi^{\bullet}}, h^{\xi}\right) \\
+ & T_{3}\left(\omega^{W}, \bar{\partial}^{Z}+v, h^{\xi^{\bullet}}\right) \\
& +\widetilde{\operatorname{ch}}\left(R^{\bullet} \pi_{3 *} \xi, h^{H\left(Z, \xi^{\bullet} \mid Z\right)}, h^{R \pi_{3 *} \xi}\right)
\end{aligned}
$$

dans $P^{S} / P^{S, 0}$ et

$$
\begin{array}{r}
T_{1}\left(\omega^{W}, h^{\xi}\right)=T_{1}\left(\omega^{W}, h^{\xi^{\bullet}}\right)+\widetilde{\operatorname{ch}}\left(F, R^{\bullet} \pi_{1 *} \xi, h^{F}, h^{R \pi_{1 *} \xi}\right) \\
-\int_{X} \operatorname{Td}\left(T X, g^{T X}\right) \widetilde{\operatorname{ch}}\left(\xi^{\bullet}, \xi, h^{\xi^{\bullet}}, h^{\xi}\right)
\end{array}
$$

dans $P^{V} / P^{V, 0}$.

D'après $(3.2),(12.3)$ et $(12.4)$, on a dans $P^{S} / P^{S, 0}$

$$
\begin{aligned}
& T_{3}\left(\omega^{W}, h^{\xi}\right)-\int_{Y} \operatorname{Td}\left(T Y, g^{T Y}\right) T_{1}\left(\omega^{W}, h^{\xi}\right) \\
& =-\int_{Z} \widetilde{\operatorname{Td}}\left(T Z, T Y, g^{T Z}, g^{T Y}\right) \operatorname{ch}\left(\xi, h^{\xi}\right) \\
& +T_{2}\left(\omega^{V}, \bar{\partial}^{Y}+v, h^{F}\right) \\
& +\widetilde{\operatorname{ch}}\left(R^{\bullet} \pi_{3 *} \xi, h^{H(Y, F \mid Y)}, h^{R \pi_{3 *} \xi}\right) \\
& \quad-\int_{Y} \operatorname{Td}\left(T Y, g^{T Y}\right) \widetilde{\operatorname{ch}}\left(F, R^{\bullet} \pi_{1 *} \xi, h^{F}, h^{R \pi_{1 *} \xi}\right) .
\end{aligned}
$$

Soit ${ }^{\prime} \mathcal{E}_{r}(\mathcal{F})$ la suite spectrale associée à $\mathcal{F}$ filtré $\operatorname{par}^{\prime} F^{p}(\mathcal{F})=\bigoplus_{p^{\prime} \geq p} \mathcal{F}^{p^{\prime}, \bullet}$ Alors pour $q>0$, on a

$$
{ }^{\prime} \mathcal{E}_{1}^{p, 0}(\mathcal{F})=F^{p}, \quad{ }^{\prime} \mathcal{E}_{2}^{p, 0}(\mathcal{F})=R^{p} \pi_{1 *} \xi, \quad{ }^{\prime} \mathcal{E}_{1}^{p, q}(\mathcal{F})={ }^{\prime} \mathcal{E}_{2}^{p, q}(\mathcal{F})=0 .
$$

Pour le bicomplexe $\mathcal{F}$, d'après la définition 10.13, et (12.6), les suites spectrales $\mathcal{E}_{r}(\mathcal{F}),{ }^{\prime} \mathcal{E}_{r}(\mathcal{F})$ dégénèrent en $\mathcal{E}_{2}(\mathcal{F}),{ }^{\prime} \mathcal{E}_{2}(\mathcal{F})$, et on a :

$$
\mathcal{E}_{2}(\mathcal{F})={ }^{\prime} \mathcal{E}_{2}(\mathcal{F})=R^{\bullet} \pi_{1 *} \xi
$$

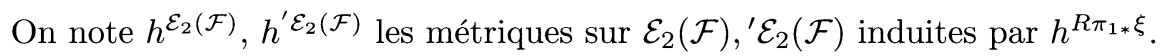

Par (10.25), pour $r=0,1$, on a

$$
E_{r}(\mathcal{F})=H^{0}\left(Y, \mathcal{E}_{r}(\mathcal{F})\right)
$$

Soit $h^{\mathcal{F}}=\bigoplus_{p, q} h^{\mathcal{F}^{p, q}}$ une métrique hermitienne sur $\mathcal{F}$. Soit $h^{\mathcal{E}_{r}(\mathcal{F})}(r=0,1)$ la métrique sur $\mathcal{E}_{r}(\mathcal{F})$ induite par $h^{\mathcal{F}}$. Soient $h^{E(\mathcal{F})}, h^{H\left(Y, \mathcal{E}_{1}(\mathcal{F})\right)}$ les métriques $\operatorname{sur} E(\mathcal{F}), E_{1}(\mathcal{F})$ induites par $g^{T Y}, h^{\mathcal{F}}, h^{\mathcal{E}(\mathcal{F})}$. 
Soient $H\left(Y, \mathcal{F}_{\mid Y}\right), H\left(Y,{ }^{\prime} \mathcal{E}_{1}(\mathcal{F})_{\mid Y}\right)$ les hypercohomologies des complexes $\left(\mathcal{F}_{\mid Y}, v+\delta\right),\left({ }^{\prime} \mathcal{E}_{1}(\mathcal{F})_{\mid Y}, v\right)$ sur $Y$. Soit $h^{H(Y, \mathcal{F} \mid Y)}$ la métrique sur $H\left(Y, \mathcal{F}_{\mid Y}\right)$ induite par $g^{T Y}, h^{\mathcal{F}}$. On rappelle qu'on a

$$
H(E(\mathcal{F}))=H\left(Y, \mathcal{F}_{\mid Y}\right)=H\left(Y,{ }^{\prime} \mathcal{E}_{1}(\mathcal{F})_{\mid Y}\right)=R^{\bullet} \pi_{3 *} \xi
$$

En procédant comme dans la preuve du théorème 11.2, dans laquelle on remplace $\bar{\partial}^{Y}$ par $\bar{\partial}^{Y}+v$, on a dans $P^{S} / P^{S, 0}$

$$
\begin{aligned}
& T_{2}\left(\omega^{V}, \bar{\partial}^{Y}+v, h^{F}\right)=T_{2}\left(\omega^{V}, \bar{\partial}^{Y}+v+\delta, h^{\mathcal{F}}\right) \\
& -\int_{Y} \operatorname{Td}\left(T Y, g^{T Y}\right) \widetilde{\operatorname{ch}}\left(\mathcal{F},{ }^{\prime} \mathcal{E}_{1}(\mathcal{F}), h^{\mathcal{F}}, h^{F}\right)+\widetilde{\operatorname{ch}}\left(R^{\bullet} \pi_{3 *} \xi, h^{H(Y, \mathcal{F} \mid Y)}, h^{H(Y, F \mid Y)}\right) .
\end{aligned}
$$

Pour le complexe $(\mathcal{F}, v+\delta)$, par le théorème 11.1 , on a dans $P^{S} / P^{S, 0}$

$$
\begin{aligned}
T_{2}\left(\omega^{V}, \bar{\partial}^{Y}+\right. & \left.+\delta, h^{\mathcal{F}}\right) \\
& =T_{2}\left(\omega^{V}, h^{\mathcal{F}}\right)+\widetilde{c h}\left(E(\mathcal{F}), H(E(\mathcal{F})), h^{E(\mathcal{F})}, h^{H(Y, \mathcal{F} \mid Y)}\right)
\end{aligned}
$$

Pour le complexe $(\mathcal{F}, v)$, en utilisant les théorèmes $11.1,11.2$ et $(12.8)$, on a dans $P^{S} / P^{S, 0}$

$$
\begin{aligned}
T_{2}\left(\omega^{V}, h^{\mathcal{F}}\right)= & \int_{Y} \operatorname{Td}\left(T Y, g^{T Y}\right) \widetilde{\operatorname{ch}}\left(\mathcal{F}, \mathcal{E}_{1}(\mathcal{F}), h^{\mathcal{F}}, h^{\mathcal{E}_{1}(\mathcal{F})}\right) \\
& +T_{2}\left(\omega^{V}, h^{\mathcal{E}_{1}(\mathcal{F})}\right)-\widetilde{\operatorname{ch}}\left(E(\mathcal{F}), E_{1}(\mathcal{F}), h^{E(\mathcal{F})}, h^{H\left(Y, \mathcal{E}_{1}(\mathcal{F})\right)}\right)
\end{aligned}
$$

Pour le complexe $\left(\mathcal{E}_{1}(\mathcal{F}), \delta\right)$, par les théorèmes 11.1 et 11.2 , on a dans $P^{S} / P^{S, 0}$

$$
\begin{aligned}
T_{2}\left(\omega^{V}, h^{\mathcal{E}_{1}(\mathcal{F})}\right)= & T_{2}\left(\omega^{V}, h^{R \pi_{1 *} \xi}\right) \\
- & \widetilde{\operatorname{ch}}\left(E_{1}(\mathcal{F}), E_{2}, h^{H\left(Y, \mathcal{E}_{1}(\mathcal{F})\right)}, h^{E_{2}}\right) \\
& +\int_{Y} \operatorname{Td}\left(T Y, g^{T Y}\right) \widetilde{\operatorname{ch}}\left(\mathcal{E}_{1}(\mathcal{F}), \mathcal{E}_{2}(\mathcal{F}), h^{\mathcal{E}_{1}(\mathcal{F})}, h^{R \pi_{1 *} \xi}\right) .
\end{aligned}
$$

Par la définition 10.15, (12.10)-(12.13), on a dans $P^{S} / P^{S, 0}$

$$
\begin{aligned}
& T_{2}\left(\omega^{V}, \bar{\partial}^{Y}+v, h^{F}\right) \\
& \quad=T_{2}\left(\omega^{V}, h^{R \pi_{1 *} \xi}\right)+\widetilde{\operatorname{ch}}\left(E_{2}, H\left(Z, \xi_{\mid Z}\right), h^{E_{2}}, h^{H(Y, F \mid Y)}\right) \\
& +\int_{Y} \operatorname{Td}\left(T Y, g^{T Y}\right)\left[\sum_{i=0}^{1} \widetilde{\operatorname{ch}}\left(\mathcal{E}_{i}(\mathcal{F}), \mathcal{E}_{i+1}(\mathcal{F}), h^{\mathcal{E}_{i}(\mathcal{F})}, h^{\mathcal{E}_{i+1}(\mathcal{F})}\right)\right. \\
& \left.-\widetilde{\operatorname{ch}}\left(\mathcal{F},{ }^{\prime} \mathcal{E}_{1}(\mathcal{F}), h^{\mathcal{F}}, h^{F}\right)\right] .
\end{aligned}
$$

BULLETIN DE LA SOCIÉTÉ MATHÉMATIQUE DE FRANCE 
Par le théorème 10.7 et $(12.7)$, on a dans $P^{V} / P^{V, 0}$

$$
\begin{aligned}
& \sum_{i=0}^{1} \widetilde{\operatorname{ch}}\left(\mathcal{E}_{i}(\mathcal{F}), \mathcal{E}_{i+1}(\mathcal{F}), h^{\mathcal{E}_{i}(\mathcal{F})}, h^{\mathcal{E}_{i+1}(\mathcal{F})}\right) \\
& =\sum_{i=0}^{1} \widetilde{\operatorname{ch}}\left({ }^{\prime} \mathcal{E}_{i}(\mathcal{F}),{ }^{\prime} \mathcal{E}_{i+1}(\mathcal{F}), h^{\prime} \mathcal{E}_{i}(\mathcal{F}), h^{\prime} \mathcal{E}_{i+1}(\mathcal{F})\right) .
\end{aligned}
$$

De $(12.5),(12.14)$ et $(12.15)$, on tire le théorème 3.11 .

\section{BIBLIOGRAPHIE}

[ABoP] Аттуан (M.F.), Botт (R.), Patodi. (V.K.). - On the heat equation and the Index Theorem, Invent. Math., t. 19, 1973, p. 279-330.

[BaFM] Baum (P.), Fulton (W.), Macpherson (R.). - Riemann-Roch for singular varietes, Publ. Math. IHES, t. 45, 1975, p. 101-146.

[BeGeV] Berline (N.), Getzler (E.), Vergne (M.). - Heat kernels and the Dirac operator. - Grundl. Math. Wiss., 298, Springer, Berlin-Heidelberg-New York, 1992.

[BerB] Berthomieu (A.), Bismut (J.-M.). - Quillen metric and higher analytic torsion forms, J. Reine angew. Math., t. 457, 1994, p. 85-184.

[B1] Bismut (J.-M.). - The Index Theorem for families of Dirac operators : two heat equation proofs, Invent.Math., t. 83, 1986, p. 91-151.

[B3] Bismut (J.-M.). - Koszul complexes, harmonic oscillators and the Todd class, J. Amer. Math. Soc., t. 3, 1990, p. 159-256.

[B4] Bismut (J.-M.). - Families of immersions, and higher analytic torsion, Astérisque, 244, 1997.

[B5] Bismut (J.-M.). - Familles d'immersions et formes de torsion analytique en degré supérieur, C.R.A.S. Paris, t. 320, série I, 1995, p. 969-974.

[BCh] Bismut (J.-M.), Cheeger (J.). - $\eta$-invariants and their adiabatic limits, J. Amer. Math. Soc., t. 2, 1989, p. 33-70.

[BGS1] Bismut (J.-M.), Gillet (H.), Soulé (C.). - Analytic torsion and holomorphic determinant bundles I, Comm. Math. Phys., t. 115, 1988, p. 49-78.

[BGS2] Bismut (J.-M.), Gillet (H.), Soulé (C.). - Analytic torsion and holomorphic determinant bundles II, Comm. Math. Phys., t. 115, 1988, p. 79-126.

[BGS3] Bismut (J.-M.), Gillet (H.), Soulé (C.). - Analytic torsion and holomorphic determinant bundles III, Comm. Math. Phys., t. 115, 1988, p. 301-351.

TOME $127-1999-\mathrm{N}^{\circ} 4$ 
[BKö] Bismut (J.-M.), Köhler (K.). - Higher analytic torsion forms for direct images and anomaly formulas, J. Alg. Geom., t. 1, 1992, p. 647-684.

[BL] Bismut (J.-M.), Lebeau (G.). - Complex immersions and Quillen metrics, Publ. Math. IHES, 74, 1991, p. 1-297.

[BS] Borel (A.), Serre (J.-P.). — Le théorème de Riemann-Roch, Bull. Soc. Math. France, t. 86, 1958, p. 97-136.

[CE] Cartan (H.), Eilenberg (S.). - Homological Algebra. - Princeton, 1956.

[CP] Charazain (J.), Piriou (A.). - Introduction à la théorie des équations aux dérivées partielles. - Gauthier-Villars, Paris, 1981.

[D] DAI (X.). - Adiabatic limits, non multiplicativity of signature and Leray spectal sequence, J. Amer. Math. Soc., t. 4, 1991, p. 265-321.

[F] Faltings (G.). - Lectures on the arithmetic Riemann-Roch Theorem, Ann. Math. Studies, Princeton University Press, 1992.

[Ge] Getzler (E.). - A short proof of the Atiyah-Singer Index Theorem, Topology, t. 25, 1986, p. 111-117.

[GrH] Griffiths (P.), Harris (J.). - Principles of Algebraic Geometry. - Wiley, New-York, 1978.

[Grot] Grothendeck (A.). - Sur quelques points d'algèbre homologique, Tôhoku Math. J., t. 9, 1957, p. 119-221.

[GS1] Gillet (H.), Soulé (C.).- Analytic torsion and the arithmetic Todd genus, Topology, t. 30, 1991, p. 21-54.

[GS2] Gillet (H.), Soulé(C.). - An arithmetic Riemann-Roch Theorem, Invent.Math., t. 110, 1992, p. 473-543.

[KM] Knudsen (P.F.), Mumford (D.). - The projectivity of the moduli space of stable curves I, Preliminaires on "det" and "div", Math. Scand., t. 39, 1976, p. $19-55$.

[Ma] Ma (Xiaonan). - Formes de torsion analytique et familles de submersions, C. R. Acad. Sciences Paris, t. 324, série I, 1997, p. 205-210.

[Ma1] MA (Xiaonan). - Formes de torsion analytique et familles de submersions II, à paraître.

[MKS] Mckean (H.), Singer (I.M.). - Curvature and the eigenvalues of the Laplacian, J. Diff. Geom., t. 1, 1967, p. 43-69.

[Q1] Quillen (D.). - Higher K-theories I., Springer Lecture Notes in Math., 341, Springer, Berlin, 1973, p. 85-147.

[Q2] Quillen (D.). - Superconnections and the Chern character, Topology, t. 24, 1986, p. 89-95.

[RS] Ray (D.B.), Singer (I.M.). - Analytic torsion for complex manifolds, Ann. of Math., t. 98, 1973, p. 154-177.

[T] TAYlor (M.). - Pseudodifferential operators. - Princeton University Press, Princeton, 1981. 